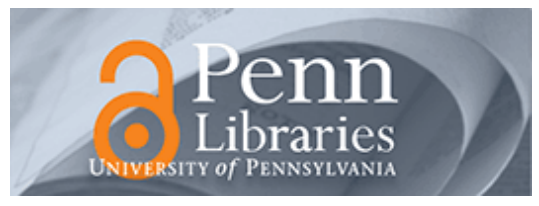

University of Pennsylvania

ScholarlyCommons

Finance Papers

Wharton Faculty Research

6-2017

\title{
Is Operating Flexibility Harmful Under Debt?
}

Dan A. Lancu

Nikolaos Trichakis

Gerry Tsoukalas

Follow this and additional works at: https://repository.upenn.edu/fnce_papers

Part of the Finance and Financial Management Commons

\section{Recommended Citation}

Lancu, D. A., Trichakis, N., \& Tsoukalas, G. (2017). Is Operating Flexibility Harmful Under Debt?.

Management Science, 63 (6), 1730-1761. http://dx.doi.org/10.1287/mnsc.2015.2415

This paper is posted at ScholarlyCommons. https://repository.upenn.edu/fnce_papers/129

For more information, please contact repository@pobox.upenn.edu. 


\title{
Is Operating Flexibility Harmful Under Debt?
}

\begin{abstract}
We study the inefficiencies stemming from a firm's operating flexibility under debt. We find that flexibility in replenishing or liquidating inventory, by providing risk-shifting incentives, could lead to borrowing costs that erase more than one-third of the firm's value. In this context, we examine the effectiveness of practical and widely used covenants in restoring firm value by limiting such risk-shifting behavior. We find that simple financial covenants can fully restore value for a firm that possesses a midseason inventory liquidation option. In the presence of added flexibility in replenishing or partially liquidating inventory, financial covenants fail, but simple borrowing base covenants successfully restore firm value. Explicitly characterizing optimal covenant tightness for all these cases, we find that better market conditions, such as lower inventory depreciation rate, higher gross margins, or increased product demand, are typically associated with tighter covenants. Our results suggest that inventory-heavy firms can reap the full benefits of additional operating flexibility, irrespective of their leverage, by entering simple debt contracts of the type commonly employed in practice. For such contracts to be effective, however, firms with enhanced flexibility and/or operating in better markets must also be willing to abide by more and/or tighter covenants.
\end{abstract}

\section{Keywords}

operating flexibility, inventory management, finance, covenants, debt

Disciplines

Finance and Financial Management 


\title{
Is Operating Flexibility Harmful Under Debt?
}

\author{
Dan A. Iancu, Nikolaos Trichakis, Gerry Tsoukalas*
}

November 12, 2015

\begin{abstract}
We study the inefficiencies stemming from a firm's operating flexibility under debt. We find that flexibility in replenishing or liquidating inventory, by providing risk shifting incentives, could lead to borrowing costs that erase more than a third of the firm's value. In this context, we examine the effectiveness of practical and widely used covenants in restoring firm value by limiting such risk shifting behavior. We find that simple financial covenants can fully restore value for a firm that possesses a mid-season inventory liquidation option. In the presence of added flexibility in replenishing or partially liquidating inventory, financial covenants fail, but simple borrowing base covenants successfully restore firm value. Explicitly characterizing optimal covenant tightness for all these cases, we find that better market conditions, such as lower inventory depreciation rate, higher gross margins or increased product demand, are typically associated with tighter covenants. Our results suggest that inventory-heavy firms can reap the full benefits of additional operating flexibility, irrespective of their leverage, by entering simple debt contracts of the type commonly employed in practice. For such contracts to be effective, however, firms with enhanced flexibility and/or operating in better markets must also be willing to abide by more and/or tighter covenants.
\end{abstract}

\section{Introduction}

Operating flexibility, i.e., the ability to adapt after uncertainty resolution, carries benefits that are widely documented in the literature. These benefits notwithstanding, flexibility may also cause inefficiencies by increasing borrowing costs due to agency issues. Shielded by limited liability, firms could use their flexibility to adapt operations in ways that benefit them at the expense of their creditors. Because debt is priced higher to reflect such risk shifting capabilities, agency costs are introduced, resulting in a loss of firm value (Myers 1977). This pitfall has been largely ignored in the OM literature, which has focused primarily on the benefits of increased flexibility, traded off against potential implementation costs.

\footnotetext{
*Iancu (daniancu@stanford.edu) is from Stanford GSB, Trichakis (ntrichakis@hbs.edu) is from Harvard Business School, Tsoukalas (gtsouk@wharton.upenn.edu) is from the Wharton School, University of Pennsylvania.
} 
In practice, however, such "flexibility-driven" agency costs can be substantial and all-the-more difficult to alleviate due to the inherent incompleteness of debt contracts, i.e., their inability to fully contract upon all future operating decisions (Aghion and Bolton 1992). For instance, while inventory-heavy firms have considerable latitude in managing their inventory levels, common debt agreements rarely prescribe or constrain such decisions in a direct way (DeAngelo et al. 2002). Covenants, i.e., requirements that borrowers must meet and that allow debtholders to take control in case of violation, ${ }^{1}$ have been suggested as a practical, albeit "roundabout" way to reduce agency costs (Smith and Warner 1979). But while existing theory clearly explains how covenants work, it does not discuss how well they work, that is, the extent to which practical covenants commonly used in debt agreements mitigate agency issues stemming from operating flexibility. By providing little guidance on how covenants should be crafted as a function of firm characteristics, existing theory is also insufficiently "operational" (Bolton 2013). The design and effectiveness of covenants thus emerge as deciding factors in unlocking the full value of flexibility, with the potential to ultimately shape a firm's operating strategy.

Our paper takes a first step towards addressing these important issues by examining them in the context of inventory management, arguably the operating capability most studied in the operations management literature. To illustrate, in this context, how firm value can be compromised by covenants that do not adequately reflect operating flexibility, we recall the now infamous example of L.A. Gear, a high growth fashion retailer whose lightning rise as a top-performing stock on the NYSE in the 1990's was matched by one of "industry's most spectacular collapses." (DeAngelo et al. 2002). Faced with a declining market and tight covenant requirements, L.A. Gear's management began to systematically liquidate inventory at fire-sale prices, in order to raise emergency cash in an attempt to avoid covenant violations and meet interest obligations. The firm gradually burned through its inventory and ultimately declared bankruptcy. ${ }^{2}$ According to DeAngelo et al. (2002),

"This large liquidation of noncash current assets was made possible by the firm's large inventory and accounts receivable beginning balances, with declines in these two items together fully accounting for the overall decline in current assets. [...] L.A. Gear's debt covenants clearly did not eliminate [management]'s ability to liquidate working capital to fund its various strategic experiments. One possible reason is that [...] workingcapital liquidations fall into a gray area that is difficult to constrain in a productive way, since they are not an outright sale of assets but are instead decisions made in the routine course of business not to replace liquid assets as they are drawn down."

This example highlights how poorly designed covenants may not adequately restrict risk shifting behavior, and may even distort operating decisions, e.g., by inducing excessive asset liquidations.

\footnotetext{
${ }^{1}$ Although debt contracts include several types of covenants (Hilson 2013), our work focuses on performance-based covenants that rely on financial metrics, which are widely used in practice (Nini et al. 2009, Roberts and Sufi 2009a).

${ }^{2}$ For a study of the relation between inventory levels and stock prices, see Gaur et al. (2014).
} 
Our study takes the perspective of an inventory-heavy firm (e.g., a retailer or manufacturer) facing uncertain demand that can issue competitively priced debt to fund its inventory investments. We model operations using the classical newsvendor paradigm, supplemented with an intermediate period during which the firm is afforded different degrees of operating flexibility to adjust inventory in response to observed sales. To isolate the role of this flexibility in generating agency costs, we abstract away from other sources of agency issues (such as information asymmetry), and preserve contract incompleteness by preventing the explicit constraining of future inventory decisions as part of the debt agreement. We instead allow debt contracts to include covenant terms commonly employed in practice, specifically, financial and borrowing base covenants (Roberts and Sufi 2009a). Debt contract terms and inventory decisions are determined endogenously. Inventory decisions remain with the firm as long as covenants are not breached, and are otherwise transferred to debtholders, who can seize and liquidate firm inventory to accelerate debt repayment.

\section{Our Contributions}

The model we develop yields two main novel insights.

1) We show that flexibility stemming from inventory management capabilities can generate surprisingly substantial agency costs for leveraged firms - potentially amounting to more than a third of firm value - when debt contracts do not include covenants (see Section 2 for details). We further find that inventory policies can be significantly distorted, both at inception and at the intermediate decision point. Specifically, we show that firms may under-order ex-ante and follow counterintuitive inventory liquidation policies ex-post, preferring continuation when operating results are weak and

liquidation when they are strong. Whereas the former effect is in line with existing theory, the latter has not previously been demonstrated (see Sections 4 and 5).

2) We show that the aforementioned agency costs and operating distortions can be fully alleviated by simple covenants widely used in practice (e.g., financial and borrowing base covenants), provided they are properly designed. We explicitly characterize the optimal types of covenants and their restrictiveness (respectively referred to as "intensity" and "tightness" in the literature, Demiroglu and James 2010) needed to restore firm value, and show how these are intrinsically related to three factors, (i) degree of operating flexibility, (ii) product parameters (e.g., margin and depreciation), and (iii) external market growth (see Sections 5 and 6).

These core insights suggest several implications and empirical predictions, discussed next.

- First, our findings send a clear message that an inventory-heavy firm contemplating operational changes to enhance flexibility, such as lead time reduction to accommodate mid-season replenishment or additional sales channels to allow for (partial) inventory liquidation, should not hesitate to finance its operations through debt; by suitably structuring its debt contracts, it can reap the full benefits of extra flexibility, irrespective of leverage. These findings nuance and even 
counter certain predictions in the finance literature that increased (production) flexibility will be accompanied by increased agency costs, tighter credit terms and reduced debt capacity (MacKay 2003). The implicit assumption underlying such predictions is that covenants used in practice are insufficiently potent to eliminate agency costs that result from a firm's operating flexibility. Our model shows that this is not necessarily the case for inventory-heavy firms. We believe this link between operating flexibility and covenant effectiveness could be empirically tested (see Section 7.2.)

- Second, we highlight the way operating capabilities and parameters are reflected in covenant design. We show that in the case of a firm that possesses a mid-season option to make all-ornothing ("0-1") liquidation decisions, such as discontinuing product lines or closing stores, simple financial covenants would suffice to fully restore firm value. When the firm has added flexibility to adjust inventory, for instance through replenishment and/or partial liquidations, as in the L.A. Gear case, financial covenants fail, leading to operating distortions and loss of value. However, we show that the addition of simple borrowing base covenants specifying an optimal haircut on the value of inventory succeeds in fully alleviating agency issues. These findings are summarized in Table 1. Our optimal characterization of covenant intensity and tightness as a function of operating parameters, moreover, yields a series of new predictions of interest to the empirical literature in accounting and finance that examines covenant tightness. In particular, we find that better market conditions, such as lower inventory depreciation rate, higher gross margins and increased product demand, typically lead to tighter covenants. These insights are further discussed in Section 7.2.

\begin{tabular}{l|c|c|c} 
Contract & $\begin{array}{c}\text { Newsvendor } \\
\text { (no flex) }\end{array}$ & $\begin{array}{c}0-1 \text { Liquidation } \\
\text { (medium flex) }\end{array}$ & $\begin{array}{c}\text { Partial Liq. / Replenishment } \\
\text { (high flex) }\end{array}$ \\
\hline Interest-rate-only & $\checkmark$ & $\boldsymbol{x}$ & $\boldsymbol{x}$ \\
Financial covenant & $\checkmark$ & $\checkmark$ & $\boldsymbol{x}$ \\
Borrowing base covenant & $\checkmark$ & $\checkmark$ & $\checkmark$
\end{tabular}

Table 1: Optimal debt contracts required as a function of the firm's operating flexibility. Check marks indicate feasibility of the contract in completely alleviating agency costs and restoring firm value. For instance, contracts that only include an interest rate are sufficient for a newsvendor (with no flexibility), but fail under added flexibility.

From a managerial perspective, our findings suggest that for debt contracts to be effective in practice, firms with enhanced flexibility and/or operating in better markets should be willing to abide by more and/or tighter covenants. This may seem counterintuitive to an operator, as (i) better markets may seem to be more "secure," and (ii) covenants clearly restrict the firm's operating discretion. Our model nevertheless predicts that optimally crafted covenants can fully mitigate agency issues, and are thus in a firm's best interest. Managerial implications are further discussed in Section 7.1.

- Extending our model to capture how competition in the lending market can affect results, we examine the extreme case in which the lender acts as a monopoly. We find inventory-heavy firms to face more covenants as competition in the lending market wanes, and covenants to no longer be 
sufficient to restore firm value.

- Our model also shows the relationship between covenant inclusion and bankruptcy risk to be more subtle than previously reported in the literature, and critically dependent on competition in the lending market and residual growth in product demand. Table 2, which illustrates this relationship, shows that it is possible that covenants are included and the equilibrium probability of bankruptcy is zero. Also, it is possible in equilibrium that the risk of bankruptcy exists but covenants are not included in the debt contract. The table highlights, moreover, that covenants become more prevalent as competition in the lending market wanes or demand growth rate increases.

\begin{tabular}{|c|c|c|}
\cline { 2 - 3 } \multicolumn{1}{c|}{} & "low" market growth & "high" market growth \\
\hline perfect competition & $\begin{array}{c}\text { covenants imply } \\
\text { bankruptcy risk }\end{array}$ & $\begin{array}{c}\text { covenants imply and are } \\
\text { implied by bankruptcy risk }\end{array}$ \\
\hline monopoly & $\begin{array}{c}\text { covenants neither imply } \\
\text { nor are implied by } \\
\text { bankruptcy risk }\end{array}$ & $\begin{array}{c}\text { bankruptcy risk implies } \\
\text { covenants }\end{array}$ \\
\hline
\end{tabular}

Table 2: Connection between bankruptcy risk and the inclusion of covenants.

The model extension for the monopolistic lending market is included in the Appendix B. Model limitations and future directions are summarized in Section 7.3.

\section{Literature Review}

Our work lies at the interface of operational and financial decision-making, an area pioneered by several recent papers. For instance, Xu and Birge (2004) and Dada and Hu (2008) extend the newsvendor model to include financing constraints, and show how these can affect the optimal order quantity or capacity choice. The focus here is on short-term financing in the form of trade credit or debt. We refer the reader to Kouvelis (2012) for a review of this literature. None of these papers consider a dynamic setting, where agency issues due to risk shifting could be relevant. Several papers in operations have discussed dynamic inventory decisions under capital constraints or leverage (e.g., Porteus 1972, Archibald et al. 2002, Gong et al. 2014), but without modeling strategic interactions or endogenizing debt contract terms. Boyabatlı et al. (2015) study the interplay of operational and financial flexility, albeit in a different context than ours.

Closer to our work, Boyabatl and Toktay (2011) examine a firm's optimal choice of flexible capacity under leverage in a static setting, finding that firms are more likely to choose flexible capacity when they use secured loans, and when they face less demand variability. Critically different from our model, flexibility does not create any agency issues in Boyabatlı and Toktay (2011), as it cannot be used to shift risk. To the best of our knowledge, only two papers in the operations literature examine the relationship between the agency costs of debt and flexibility. 
Chod and Zhou (2014) study a newsvendor-style firm that first secures debt, and then chooses whether to invest in flexible or inflexible (but cheaper) resources. Risk shifting arises when the latter option is chosen, as inflexible resources are "riskier" than flexible ones. Thus, in this static model, increased (resource) flexibility does not accentuate agency issues, but rather reduces them. In contrast, our emphasis is on studying the risk shifting potential that operating flexibility can introduce, which can only be captured in a dynamic setting. Finally, Chod (2015) study the role of trade credit in alleviating the agency costs of debt. The focus is on a newsvendor-style firm that first secures funding - through bank debt or trade credit - and then places orders for two types of products. The author shows how agency costs arise under (interest-only) debt financing, as the firm can order the "riskier" product once debt is in place. In contrast, when trade credit is provided by a single supplier who can directly contract upon the individual order quantities, agency costs are fully alleviated. While both Chod (2015) and the present paper discuss agency costs arising from operating flexibility, the papers differ dramatically in focus: the former looks at trade credit, while we examine the efficiency and optimal design of common financial contracts (with covenants).

On a broader level, our work is related to a large body of accounting, finance and economics literature that deals with agency issues and contractual incompleteness. Seminal works include Jensen and Meckling (1976), Myers (1977), Grossman and Hart (1986), and Aghion and Bolton (1992). Smith and Warner (1979) were the first to describe how covenants can be used to counter agency issues, whereas Leland (1994) argued how net worth covenants can mitigate agency issues occasioned by risk shifting. The literature on the role of covenants in alleviating agency and incompleteness is vast, and we make no attempt to survey it here - we refer the interested reader to Bolton and Dewatripont (2004), Tirole (2006), and references therein.

Several papers within this stream study agency costs derived from different types of flexibility, see, e.g., Childs et al. (2005), Leland (1998), Moyen (2007), Titman et al. (2004). The focus is on comparative statics to assess the directional impact of flexibility on such costs and/or numerical studies to quantify the magnitude of costs. Mello and Parsons (1992) and Manso (2008) study special forms of operating flexibility (captured through switching costs), with the latter deriving upper bounds on the agency costs. None of these papers look at the effectiveness of optimally designed covenants in alleviating agency costs, which is the primary focus of our paper.

Closer to our work, some recent papers focus on quantifying agency costs and covenant effectiveness. Gamba and Triantis (2014) compares the effectiveness of different types of covenants used in practice through a detailed numerical study, showing that covenants could restore anywhere from $0 \%$ to $90 \%$ of the firm value loss. Matvos (2013) develops a structural model to measure which types of covenants are most effective in practice, finding that covenant presence is essential, but - once covenants are chosen - the benefits of "fine-tuning" them is marginal. In contrast, we find that significant firm value could be at stake if covenant tightness is not properly set (see Section 2). Neither of these papers looks at optimal covenant design. Berlin and Mester (1992), 
Sridhar and Magee (1997) and Gârleanu and Zwiebel (2009) study the relation between optimal covenant design/tightness and renegotiation, which becomes important under information frictions. We instead study how to optimally design covenants in order to mitigate agency issues caused by (varying degrees of) operating flexibility, which, to the best of our knowledge, has not been looked at before. Moreover, the aforementioned papers do not focus on quantifying covenant effectiveness.

In Section 7.2, we review several empirical papers in the literature studying covenant tightness, intensity, effectiveness that our work nuances and is either aligned with, or even counters.

\section{Quantifying Agency Costs}

Before introducing the modeling details and analysis, it will be useful to gain a better understanding of the magnitude of the effects discussed in the introduction. Are flexibility-driven agency costs a first order effect in our setting? To assess this, we first measure the potential value created by inventory flexibility, and then quantify how much of this value can be erased under leverage.

We rely on a base case newsvendor model, denoted by $\mathcal{N}$, with a single selling season $[0,2]$. To introduce operating flexibility, we extend this model by splitting the selling season into two periods, $[0,1]$ and $[1,2]$, and in the spirit of Fisher et al. (2001), we allow an opportunity to adjust inventory in response to observed sales inbetween. We refer to this model as the "flexvendor." As alluded to in the introduction, we study different degrees of the flexvendor's flexibility in adjusting inventory. In this section, we consider the highest degree of flexibility, where the flexvendor can fully adjust inventory either by partial liquidation or replenishment, and study the following two variations:

$\mathcal{F}$ : a flexvendor with ample capital, who requires no debt financing.

$\mathcal{F}^{\text {lev}}$ : a leveraged flexvendor, who primarily relies on debt financing through competitivelypriced debt contracts that only include an interest rate.

The flexvendor model is rooted in reality, and motivated by the decision process faced by firms carrying inventory that depreciates in value over time. In particular, we are inspired by the case of Hewlett Packard (HP), which attempted to enter the tablet market in the summer of 2011, with two TouchPad devices (16GB and 32GB versions) that were based on HP's proprietary webOS software. As of August 2011, the two devices retailed for $\$ 399.99$ and $\$ 499.99$, respectively (Rapaport and Tracer 2011). Over the course of the month, HP's sales were extremely disappointing, while its competitors were rapidly gaining market share. It soon became apparent that HP's software could become obsolete, unable to compete with the Apple and Android ecosystems. As a result, towards the end of August 2011, HP decided to completely liquidate its webOS line of tablets, at fire-sale prices. After some price experimentation, the retail liquidation prices were set at $\$ 249.99$ and $\$ 299.99$, respectively (Wikipedia 2011).

We rely on this example to calibrate the flexvendor's operating parameters, namely prices and unit cost. As HP's primary distribution channel was through wholesale to its retail partners 
(e.g., Best Buy), we need estimates of HP's wholesale prices during normal operations, and during liquidation. According to Damodaran (2015), retail consumer electronics margins averaged 18.38\% in 2011. This implies tablet wholesale prices of around $\$ 338$ (16GB) and $\$ 422$ (32GB), during normal operations, and $\$ 211$ (16GB) and $\$ 253$ (32GB) during the liquidation. Furthermore, HP's unit costs - including parts and labor-were estimated at around $\$ 306.15$ (16GB) and $\$ 328.15$ (32GB), (Wikipedia 2011). Averaging across the two devices, we obtain an average unit cost of around $83 \%$ of wholesale, and an average liquidation price of around $61 \%$ of wholesale.

Accordingly, we normalize the flexvendor selling price to 1 , and set the unit ordering cost to 0.83 (in both periods) and the liquidation value to 0.61 at time 1 . To capture the webOS technological obsolescence, there is no salvage value at time 2. Further, we assume first period demand is uniformly distributed between [0,10], and the second period demand - conditioned on the first period realized demand being $d_{1}$-is uniformly distributed in $\left[0,2 d_{1}\right]$. $\mathcal{F}^{\text {lev }}$ uses equity of 0.2 , so that the purchase is primarily financed by debt. The profits are also normalized to ease exposition, such that the expected profits of $\mathcal{N}$ are set at 100, see Figure 1(a).

Comparing the newsvendor $\mathcal{N}$ in Figure 1(a) to the flexvendor $\mathcal{F}$ in Figure 1(b) highlights the value of inventory flexibility: the flexvendor extracts profits of 235 , a $135 \%$ increase compared to the newsvendor. This also represents the "first best" outcome for the flexvendor, i.e., the total profits or firm value achievable with flexibility, and without any financial frictions.

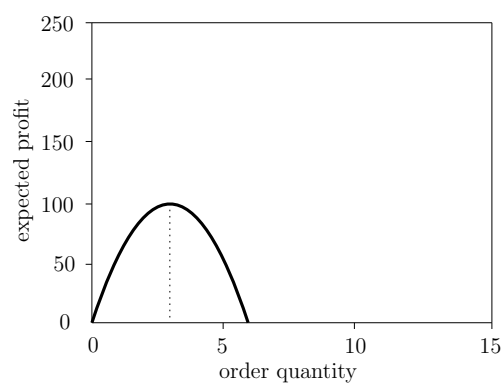

(a) Newsvendor $\mathcal{N}$

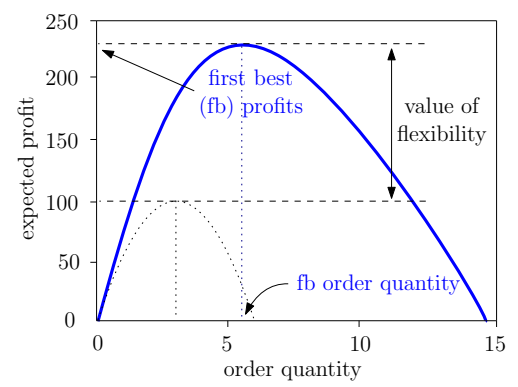

(b) Flexvendor $\mathcal{F}$

Figure 1: The value of operating flexibility: (a) Newsvendor vs. (b) Flexvendor

Introducing debt financing leads to agency issues, as the leveraged flexvendor - shielded by limited liability - can use his additional operating flexibility to adjust the inventory level in risky ways, e.g., by carrying too much inventory into the second period (this intuition is formalized in Section 4). This leads to some rather striking results. If debtholders do not anticipate this risk shifting potential, the leveraged flexvendor could expropriate wealth from them, and beat first best, as shown in Figure 2(a). Under equilibrium pricing, debtholders fully anticipate risk shifting and increase borrowing rates in response, which introduces agency costs, as shown in Figure 2(b). Consequently, the leveraged flexvendor achieves profits of 160, which are $32 \%$ lower than the flexvendor's first best. This loss is substantial, suggesting that flexibility-driven agency costs can be a dominant 
effect in our setting, ${ }^{3}$ potentially shaping operations strategy. For instance, consider the situation where the newsvendor would need to invest 100 to obtain the reordering/liquidation flexibility of the flexvendor. In the absence of financial frictions, such an investment would be fully justified, yielding a net benefit of $135-100=35$. However, under financial frictions, the net benefit becomes $60-100=-40$, rendering the investment unattractive.

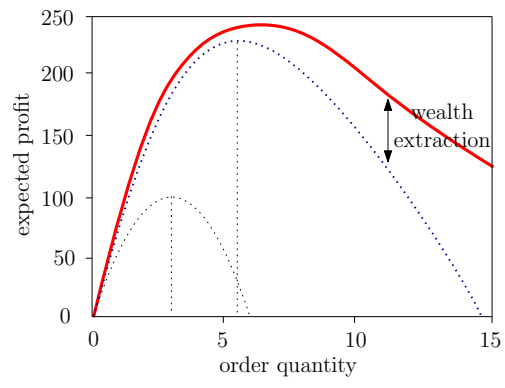

(a) Risk shifting example

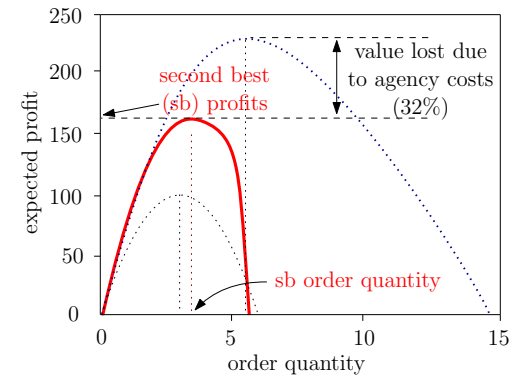

(b) Agency costs created

Figure 2: How a leveraged flexvendor could shift risk to extract wealth from non-anticipating debtholders (a), and how agency costs are created when debtholders anticipate this behavior in equilibrium (b), in the context of interest-only debt contracts.

To evaluate the potential of covenants in alleviating agency costs, we now consider the case where the debt contract for the leveraged flexvendor includes a simple financial covenant of varying tightness, evaluated between the two periods. In particular, the covenant requires that the cash generated from sales in the first period meet a minimum threshold. The covenant becomes tighter as the required threshold increases. Upon covenant violation, the decision rights are transferred to the debtholders. Figure 3 illustrates the effectiveness of a covenant with (a) low, (b) medium or (c) high tightness in mitigating the value losses. It is interesting to note that the three covenants reduce agency costs to (a) 13\%, (b) 4.5\%, and (c) 7.5\%, respectively, so that effectiveness is nonmonotonic in covenant tightness. The intuition behind this is that if the covenant is too loose, risk shifting activities are not adequately restricted. However, if the covenant is too tight, then the debtholders essentially control the firm after the first period, and take decisions that are also inefficient. We discuss this extensively in Section 5 .

While this simple example sets the stage, it leaves much unanswered: How effective are financial covenants in mitigating flexibility-driven agency costs and restoring firm value? Is one covenant enough, or are more needed? How should the covenant intensity and tightness be structured as a function of the product (price, margin, salvage), the market (demand growth), and the firm's operating capabilities (reordering and liquidation options)? The rest of the paper seeks answers to

\footnotetext{
${ }^{3}$ We conducted several robustness checks, by varying all parameters in this model, and the magnitude of the effect persisted. We note that these costs are larger than values reported in other studies. For instance, Chod (2015) reports agency costs of less than $8 \%$ in a single-period inventory model, while Gamba and Triantis (2014) report costs as large as $18 \%$ (albeit in a very different model specification).
} 


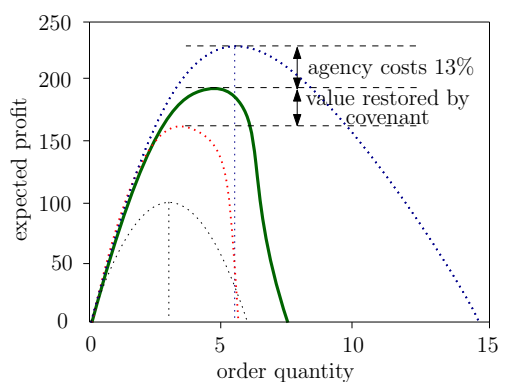

(a) low tightness

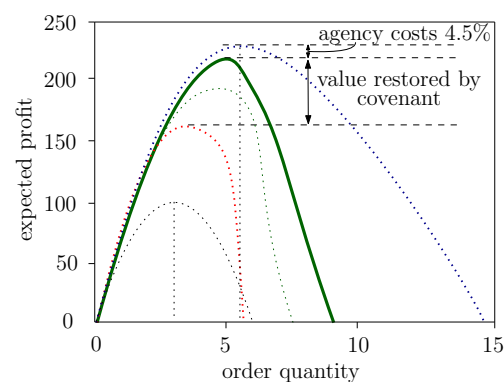

(b) average tightness

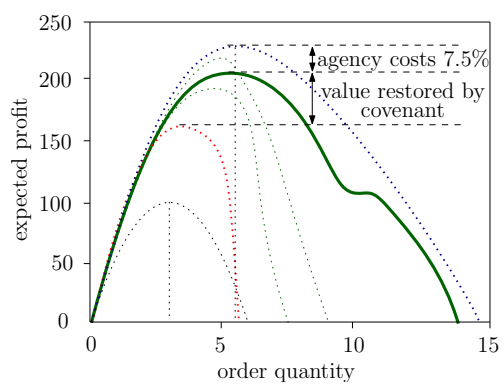

(c) high tightness

Figure 3: Quantifying how much value covenants can restore for the leveraged flexvendor, as a function of tightness.

these questions.

\section{Model}

We introduce the flexvendor model by considering a newsvendor who has an early exit option, i.e., an intermediate time-step at which all remaining inventory can be liquidated, before fully depreciating (we return to study the flexvendor with replenishment flexibility in Section 6).

\subsection{Flexvendor With a Liquidation Option}

We consider a setting in which a firm can purchase a single type of product at time $t=0$, at unit cost $c>0$. The number of units to purchase is denoted by $q$; full payment of $c q$ is due upon delivery of the purchased units, which occurs with zero lead time. There are two selling periods, over which products are sold at price $p=1>c$. At $t=1$, the first period random demand $D_{1}$ is realized, and fulfilled to the largest extent possible from the initial inventory of $q$ units. We assume that $D_{1}$ has non-negative support, cumulative distribution function (c.d.f.) $F_{1}$ and probability density function (p.d.f.) $f_{1}$, and denote a realization with $d_{1}$.

At this point, a participating firm faces two options: it can either liquidate (e.g., salvage) the remaining inventory $\left(q-d_{1}\right)^{+}$(if any) at unit price $s<c$ and exit the market immediately, or continue operating for a second selling period. The decision to continue or liquidate, denoted by $\ell \in\{0,1\}$, is based on the information set at $t=1$, which consists of the realized demand $d_{1}$.

If the firm chooses to continue, i.e., $\ell=0$, the second period demand $D_{2}$ is realized and fulfilled at $t=2$, to the largest extent possible. We assume that $D_{2}$ depends on $d_{1}$, specifically, $D_{2} \mid\left\{D_{1}=d_{1}\right\}=\left(\frac{M-1}{2} d_{1}\right) Z$, where $Z$ is a non-negative random variable with unit mean, and $M>1$ is a parameter determining the demand growth rate, or market strength. In particular, since $\mathbb{E}\left[D_{2} \mid D_{1}=d_{1}\right]=\frac{M-1}{2} d_{1}$, demand is projected to increase if $M \geq 3$, and decrease otherwise. We refer to these cases as follows.

Definition 1. When $M \geq 3$ ( $M<3)$, we say that the market is non-shrinking (shrinking). 
Unmet demand is lost without any penalty in both periods, and any remaining inventory is disposed of, without salvage value or cost. ${ }^{4}$ For simplicity, we assume a zero discount rate throughout the analysis. The value extracted from such a market under an initial order $q$ is thus given by the firm's expected profit,

$$
V(q)=\mathbb{E}_{D_{1}}\left[\min \left(D_{1}, q\right)+\max _{\ell \in\{0,1\}}\left\{\ell \cdot s\left(q-D_{1}\right)^{+}+(1-\ell) \cdot \mathbb{E}_{D_{2} \mid D_{1}}\left[\min \left(D_{2},\left(q-D_{1}\right)^{+}\right)\right]\right\}\right]
$$

and the firm chooses $q$ at $t=0$ so as to maximize $V(q)$. Let $V^{\mathrm{fb}} \stackrel{\text { def }}{=} \max _{q \geq 0} V(q)$ denote the optimal extracted value, and $q^{\mathrm{fb}}$ denote the associated optimal order quantity, henceforth referred to as first best.

\subsection{Leveraged Flexvendor}

A firm (e.g., a retailer) $\mathcal{R}$ operates in the setting described above. When the firm's initial capital $x_{0}$ is insufficient to cover the inventory cost $c q$, it can obtain debt financing from a perfectly competitive lending market. More precisely, a bank $\mathcal{B}$ ("she") can extend a loan for the amount $w \stackrel{\text { def }}{=}\left(c q-x_{0}\right)^{+}, 5$ under a contract with the following (T)erms and (C)ontingencies.

[T1] (Interest rate and repayment) Payment of principal plus interest charges (at rate $r$ ) is due at $t=2$. For ease of exposition, we consider $R=1+r$ in the remainder of the paper, and refer to it simply as the interest rate. The amount due at $t=2$ is then $R w$.

[T2] (Collateral and withdrawal) The bank obtains perfect security interest in the entire inventory (to be) purchased by the firm. All cash generated by selling the inventory is remitted to a lockbox account controlled by the bank, and the firm is unable to access the funds until the entire loan proceeds $R w$ are fully repaid, at $t=2$. In other words, the lockbox account has a zero withdrawal limit.

[C1] (Financial covenant) In period $t=1$, the firm's cash flow $\min \left(d_{1}, q\right)$ is required to exceed a threshold $\delta$. Failure to abide by the covenant is considered an event of default, which gives the bank full control rights, as well as the ability to request immediate repayment of all due principal plus interest, $R w$.

[C2] (Borrowing base covenant) In period $t=1$, the firm's borrowing base is calculated using an advance rate of 1 for cash and $\alpha \in[0,1)$ for inventory, and is thus equal to $\min \left(d_{1}, q\right)+\alpha s(q-$ $\left.d_{1}\right)^{+}$. The borrowing base is required to exceed a threshold $\beta$, and as with [C1], failure to conform triggers an event of default, and carries the same repercussions.

\footnotetext{
${ }^{4}$ The assumption of zero salvage at $t=2$ simplifies exposition, without affecting our structural results and insights.

${ }^{5}$ We implicitly assume that all available equity is used to purchase the inventory at $t=0$. Absent other considerations, this is without loss (Kouvelis and Zhao 2012).
} 
All contracts we consider include terms [T1-2]. We denote the set of contracts that include no contingencies by $\mathbb{K}^{\emptyset}$, and refer to such contracts as interest-rate-only contracts. Similarly, let $\mathbb{K}^{\mathrm{F}}$ be the set of contracts that include a financial covenant $[\mathrm{C} 1]$ only, and $\mathbb{K}^{\mathrm{B}}$ the set of contracts that include a borrowing base covenant [C2] only. It is worth noting that a borrowing base covenant is more general than a financial (i.e., cash flow based) covenant, since the latter can always be replicated through the former by taking $\alpha=0$ and $\beta=\delta$. As such, $\mathbb{K}^{\emptyset} \subset \mathbb{K}^{\mathrm{F}} \subset \mathbb{K}^{\mathrm{B}}$.

We consider separate model specifications, in which contracts are chosen from either $\mathbb{K}^{\emptyset}, \mathbb{K}^{\mathrm{F}}$ or $\mathbb{K}^{\mathrm{B}}$. To simplify notation, we denote any contract with $\kappa$.

\subsubsection{Timing of Events}

We study the game under perfect and symmetric information between the two players, $\mathcal{R}$ and $\mathcal{B}$. At $t=0$, the firm chooses an order quantity $q$, and the bank chooses the terms (and contingencies) in the loan contract $\kappa$.

At $t=1$, demand $d_{1}$ is revealed to both players and fulfilled, generating a cash flow of $\min \left(d_{1}, q\right)$ for the firm. As described above, the firm has the option of continuing operating for a second selling period, or liquidating any leftover inventory at $s$ and exiting the market. If the latter option is chosen, the firm uses generated cash flow and liquidation revenues, equal to $\min \left(d_{1}, q\right)+s\left(q-d_{1}\right)^{+}$, to repay its debt of $R w$. Let $\ell_{\mathcal{R}}: \mathbb{R} \times \mathbb{R} \rightarrow\{0,1\}$ denote the firm's liquidation policy, with $\ell_{\mathcal{R}}\left(q, d_{1}\right)=1$ if and only if the firm liquidates when the initial inventory is $q$, and $D_{1}=d_{1}$.

If the firm chooses to continue operating and the contract includes a covenant, then the bank evaluates the covenant requirement. If it is breached, the bank has the option of forcing liquidation in order to secure full payment of the debt. In case the bank exercises this option, she seizes remaining inventory and liquidates it at unit price $s$. All proceeds from sales and liquidation are first used towards debt servicing. If the bank is made whole, the remaining revenues are returned to the firm. Similar to our previous notation, let $\ell_{\mathcal{B}}: \mathbb{R} \times \mathbb{R} \rightarrow\{0,1\}$ denote the liquidation policy of the bank. Note that, unless a covenant is included in the contract and it is breached, the bank does not have any right to influence the liquidation/continuation decision.

When the firm chooses to continue and the bank does not force liquidation, the second period demand $D_{2}$ is realized and filled to the largest extent possible. At $t=2$, the generated sales revenues from the two periods are first used to repay the debt $R w$, and the firm keeps any remaining cash and exits the market.

In this setting, the firm's and the bank's cash flows at the end of the game under the liquidation event $^{6} \mathscr{L}$ (irrespective of the party choosing that option) and continuation event $\mathscr{C} \stackrel{\text { def }}{=} \mathscr{L}^{c}$ are

\footnotetext{
${ }^{6}$ More formally, $\mathscr{L} \stackrel{\text { def }}{=}\left\{\ell_{\mathcal{R}}\left(q, D_{1}\right)=1\right\} \cup\left\{\mathcal{V} \cap\left\{\ell_{\mathcal{B}}\left(q, D_{1}\right)=1\right\}\right\}$, where $\mathcal{V}$ is the event corresponding to a covenant breach, i.e., $\mathcal{V} \stackrel{\text { def }}{=}\left\{\kappa \in \mathbb{K}^{\mathrm{F}} \cap\left\{\min \left(q, d_{1}\right)<\delta\right\}\right\} \cup\left\{\kappa \in \mathbb{K}^{\mathrm{B}} \cap\left\{\min \left(q, d_{1}\right)+\alpha s\left(q-d_{1}\right)^{+}<\beta\right\}\right\}$.
} 
respectively given by

$$
\begin{aligned}
& X_{\mathcal{R}, \mathscr{L}}\left(q, D_{1}\right)=\left(\min \left(D_{1}, q\right)+s\left(q-D_{1}\right)^{+}-R w\right)^{+}, \quad X_{\mathcal{R}, \mathscr{C}}\left(q, D_{1}, D_{2}\right)=\left(\min \left(D_{1}+D_{2}, q\right)-R w\right)^{+}, \\
& X_{\mathcal{B}, \mathscr{L}}\left(q, D_{1}\right)=\min \left\{R w, \min \left(D_{1}, q\right)+s\left(q-D_{1}\right)^{+}\right\}, \quad X_{\mathcal{B}, \mathscr{C}}\left(q, D_{1}, D_{2}\right)=\min \left\{R w, \min \left(D_{1}+D_{2}, q\right)\right\} .
\end{aligned}
$$

For the firm, both expressions have a floor at 0, reflecting its limited liability. For clarity, we refer to the game at $t=1$ that determines whether $\mathscr{L}$ or $\mathscr{C}$ occurs as subgame $\mathbf{S}$. The pure subgame perfect equilibrium actions can be characterized via backward induction. When $\kappa \in \mathbb{K}^{\emptyset}$, the game only entails choices by the firm. When $\kappa \in \mathbb{K}^{\mathrm{F}} \cup \mathbb{K}^{\mathrm{B}}$, the outcome of the subgame $\mathbf{S}$ can be viewed as a Stackelberg game, with the firm leading by choosing $\ell_{\mathcal{R}}$ and the bank following by choosing $\ell_{\mathcal{B}}$ (see Figure $4(\mathrm{~b})$ ).

At $t=0$, the expected profits of the two players can then be compactly expressed as

$$
\begin{aligned}
\pi_{\mathcal{R}}(q, \kappa) & =\mathbb{E}\left[X_{\mathcal{R}, \mathscr{L}}\left(q, D_{1}\right) \mathbb{1}\{\mathscr{L}\}+X_{\mathcal{R}, \mathscr{C}}\left(q, D_{1}, D_{2}\right) \mathbb{1}\{\mathscr{C}\}\right]-x_{0}, \\
\pi_{\mathcal{B}}(q, \kappa) & =\mathbb{E}\left[X_{\mathcal{B}, \mathscr{L}}\left(q, D_{1}\right) \mathbb{1}\{\mathscr{L}\}+X_{\mathcal{B}, \mathscr{C}}\left(q, D_{1}, D_{2}\right) \mathbb{1}\{\mathscr{C}\}\right]-w
\end{aligned}
$$

where $\mathbb{1}$ is an indicator function. The firm chooses the order quantity $q$ so as to maximize its expected profit $\pi_{\mathcal{R}}(q, \kappa)$, and the bank chooses the debt contract terms $\kappa$ so as to break even in expectation, i.e., $\pi_{\mathcal{B}}(q, \kappa)=0$. The sequence of all events is illustrated in Figure 4.

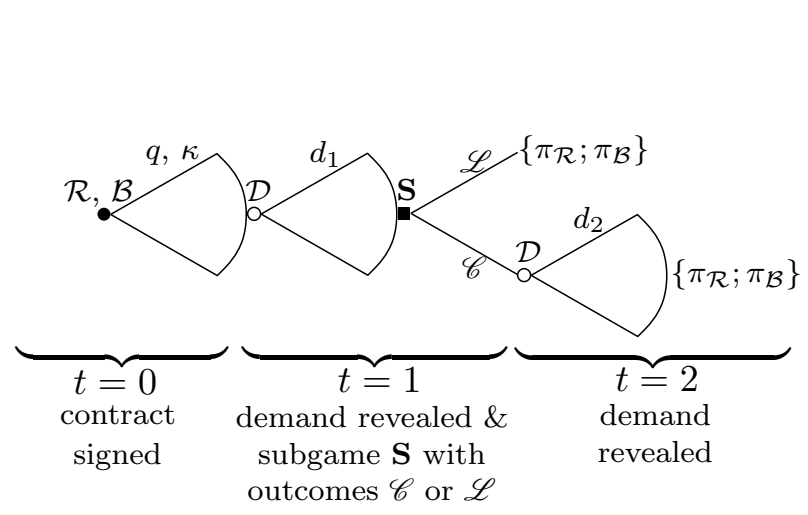

(a) Game under perfect competition

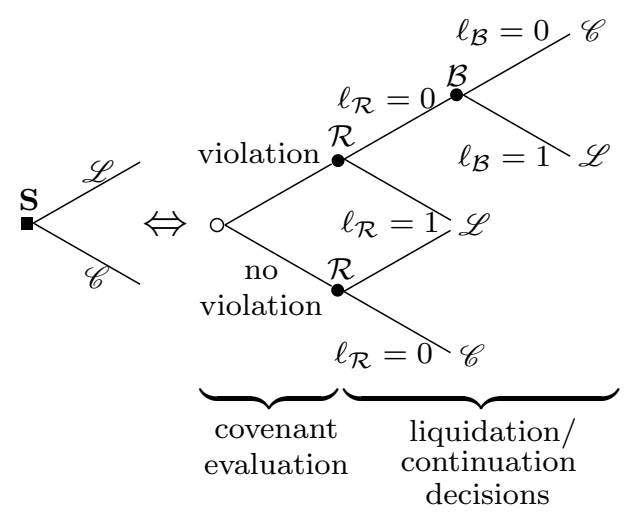

(b) Subgame $\mathbf{S}$

Figure 4: Game under perfect competition and subgame $\mathbf{S}$ for $\kappa \in \mathbb{K}^{\mathrm{F}} \cup \mathbb{K}^{\mathrm{B}}(\mathcal{R}=$ Firm, $\mathcal{B}=$ Bank, $\mathcal{D}=$ Demand $)$.

\section{Assumptions for Analysis}

For the sake of analytical tractability, we also introduce the following simplifying assumptions. We relax these in Section 6.

Assumption 1. The random variable $Z$ that drives second period demand follows a two-point distribution, taking values 0 and 2 with equal probability. 
The two-point distribution introduces an adverse $\left(D_{2}=0\right.$, for $\left.Z=0\right)$ and a favorable scenario $\left(D_{2}=(M-1) d_{1}\right.$, for $\left.Z=2\right)$ for the second period demand. Our choices of zero demand in the adverse scenario and equally likely scenarios allow us to ease exposition, without affecting the validity of the structural results.

Under Assumption 1, the probability of selling a unit of inventory over the second period is at most $1 / 2$, and the corresponding expected revenues are at most $1 / 2$ as well, since the selling price is 1 . On the other hand, liquidation yields revenues of $s$ per unit at $t=1$. It is easy to see that, unless $s<1 / 2$, the firm would never prefer to continue in the second period, but rather behave as a classical newsvendor who sells over one period and then liquidates (or salvages) his leftover inventory. To circumvent this degeneracy, we introduce the following assumption:

Assumption 2. The liquidation price satisfies $s<1 / 2$.

It is hardly surprising that an upper bound on the value of $s$ is needed to eliminate trivial cases when liquidation is always preferred.

\subsection{Discussion of Modeling Assumptions}

Business cycle. Our model extends the classical newsvendor framework by providing a liquidation option at an intermediate point. This can correspond to a discontinuation of a division or product, or a going out of business sale (Craig and Raman 2013). Paralleling the standard "salvaging" assumption in the newsvendor setup, we assume that the entire leftover inventory at $t=1$ can be liquidated at a price $s$ that is a-priori known, and independent of the quantity and first period demand. While simplistic, ${ }^{7}$ this assumption adequately captures the mechanisms at play in our setting, and becomes even more sensible when the bank is also endowed with a liquidation option. In practice, such liquidations are typically contracted to an external party, such as Gordon Brothers Group, Hilco Global, ES Group, etc. Such a liquidation house provides binding inventory appraisals upfront (i.e., in period $t=0$ ), and, in case of liquidation, purchases the entire inventory at a fixed per-unit price, which reflects a pre-determined haircut on the original appraised value. Thus, any remaining liquidation risk beyond this haircut is essentially transferred to the liquidation house (see Craig and Raman 2013 for more details).

Demand distribution. Requiring $D_{1}$ and $D_{2}$ to be correlated is standard in the literature (Fisher and Raman 1996). Our assumption of a discrete second period demand distribution has been widely used in the literature in similar multi-period game-theoretic models (e.g., see Bolton and Dewatripont 2004 and references in our literature review in Section 1). Despite its simplicity, the

\footnotetext{
${ }^{7}$ Cachon and Kok (2007) recognize that the effective salvaging price depends on remaining inventory, and discuss appropriate ways for estimating this price. There is also an extensive literature in revenue management concerned with optimal markdown, the practice of dynamically adjusting prices for remaining inventory at the end of a selling season, for the purpose of freeing valuable shelf space (see, e.g., Talluri and Van Ryzin 2004 and Phillips 2005).
} 
two-point distribution is sufficiently rich to capture the main trade-offs driving the players' dynamic decisions, and the results derived are robust, as discussed in Section 6 and Section C.1.

Exogenous retail price. We note that the flexvendor in our model is assumed to be price-taking, as in the classical newsvendor setting.

Collateral. Assuming the loan to be secured allows us to abstract away from potential complexities related to the bank's seniority in the capital structure.

No loan limit. In our model, given that the interest rate is determined endogenously, a loan limit would be superfluous and is thus omitted. This is standard in the finance and the OM literatures (Boyabatlı and Toktay 2011, Gârleanu and Zwiebel 2009, Myers 1977, Xu and Birge 2004).

No cash diversion/dividends. The presence of a lockbox account and the implicit assumption of no dividend payment are both common requirements in loan agreements (see Chapter 7 of Hilson 2013, pages 14 and 19). In our model, this allows abstracting away any additional agency issues related to cash diversion, and focusing on agency issues exclusively due to operating flexibility.

No additional financing. Allowing the firm no access to other external capital (e.g., by new equity or debt issuance) during the life of the contract is standard in the literature (see, e.g., Chod and Zhou 2014, Gârleanu and Zwiebel 2009, Myers 1977, Xu and Birge 2004). This allows focusing on the relationship between the two agents.

Contingencies. Since the goal of our paper is to quantify the effectiveness of simple and practical covenants in alleviating agency issues, the debt contracts we consider only include financial or borrowing base covenants. Together with pricing grids, these are the most commonly used forms of contingencies in practice, as documented by numerous empirical papers (Roberts and Sufi 2009a).

Financial covenant. Financial covenants involve various metrics, such as cash-flow-to-debt ratio, net worth, interest rate coverage, EBIT to interest ratio, etc. (see, e.g., Dichev and Skinner 2002, Roberts and Sufi 2009b, and pages 2-4 in Chapter 7 of Hilson 2013). In our base model, it turns out that all such covenants translate into a minimum threshold requirement on the cash flow or, equivalently, on the first period demand $d_{1}$ (note that the covenant can never be breached upon stock-out). ${ }^{8}$ The choice of a particular type of financial covenant is therefore without loss of generality, so that we henceforth base our analysis and discussion on a minimum cash flow threshold $\delta$ that controls the covenant tightness. Finally, entitlement to immediate debt payment upon a covenant breach is routinely included in contracts (see page 17 in Chapter 7 of Hilson 2013).

Borrowing base covenant. The borrowing base is the value assigned to the borrower's pledged assets, calculated by applying particular advance rates against each type of collateral. These rates (i.e.,

\footnotetext{
${ }^{8}$ For instance, consider a covenant requiring the net worth to be higher than a threshold $\tau$. For $d_{1}<q$, debt at $t=1$ is equal to $R w$, and equity is equal to $d_{1}+s\left(q-d_{1}\right)$. The covenant then equivalently requires demand to be higher than a threshold, i.e., $d_{1}>\frac{\tau+R w-s q}{1-s}$.
} 
"hair-cuts") are chosen by the lender as additional risk controls, reflecting the riskiness of each asset class. Our assumption that cash and inventory have advance rates of 1 and $\alpha$, respectively, is consistent with common practice, where accounts receivable advance rates are often 90\%-100\%, while inventory rates range from 50\% to 90\% (Buzacott and Zhang 2004, CH 2014). Most secured contracts include contingencies requiring the borrowing base to exceed the size of the outstanding debt throughout the life of the loan. We note that the covenant used in our model is slightly more general, by requiring the borrowing base at $t=1$ to exceed a value $\beta$, which is not necessarily equal to the outstanding debt. We retain the terminology of "borrowing base covenant" for simplicity of exposition. $\beta$ controls the tightness of the covenant. A borrowing base requirement at $t=0$ would essentially be equivalent to a loan limit, which is superfluous as per our discussion above.

Bankruptcy costs and procedures. In the event of default, we only consider Chapter 7 bankruptcy ("liquidation"), but not Chapter 11 ("reorganization"), which would involve having to model the renegotiation process between the parties. Note also that, in view of our assumption of perfect and symmetric information, a renegotiation process would be superfluous (see Gârleanu and Zwiebel 2009). We also ignore bankruptcy costs. For a discussion on the effects of non-zero bankruptcy costs and reorganization, we refer the reader to Birge and Yang (2013) and Birge et al. (2015).

\section{Liquidation Policies}

We analyze the players' optimal liquidation policies in subgame $\mathbf{S}$, at the intermediate time $t=1$. To provide a meaningful comparison, we start by deriving the first best policy.

\subsection{First Best Liquidation Policy}

When the order quantity is $q$, and the first period demand realization is $D_{1}=d$, the first best policy can be readily derived as the optimal liquidation decision $\ell$ in (1).

Lemma 1 (First Best). The first best liquidation policy is a threshold one,

$$
\ell^{\mathrm{fb}}(q, d)=\mathbb{1}\left\{d<d^{\mathrm{fb}}(q)\right\}, \text { where } d^{\mathrm{fb}}(q) \stackrel{\text { def }}{=} \frac{s q}{\frac{M-1}{2}+s}
$$

Such a threshold policy is consistent with intuition and hardly surprising. Many studies dealing with dynamic decisions under debt either de facto assume or derive such policies as optimal (see, e.g., Babich and Sobel 2004, Gigler et al. 2009, Hart and Moore 1998, Morellec 2001, Swinney and Netessine 2009). Furthermore, as one might intuitively expect, the threshold $d^{\mathrm{fb}}$ is increasing in $q$ and $s$ and decreasing in $M$, confirming that the propensity to liquidate increases with order quantity and liquidation value, but decreases with the second period market strength. 


\subsection{Leveraged Firm}

Under debt, the firm's liquidation decisions generally depart from first best. The optimal policy turns out to critically depend on whether the order quantity $q$ exceeds a particular threshold $q_{\mathbb{D}}$, given by

$$
q_{\mathbb{D}} \stackrel{\text { def }}{=} \begin{cases}\frac{R x_{0}}{R c-\frac{2 s}{M-1+2 s}} & \text { if } M \geq \tilde{M} \text { and } s<s_{\mathbb{D}}^{1} \\ \frac{R x_{0}}{R c-\frac{s M}{M+s-1}} & \text { if } M<\tilde{M} \text { and } s<s_{\mathbb{D}}^{2} \\ \infty & \text { otherwise }\end{cases}
$$

where $\tilde{M} \stackrel{\text { def }}{=} 2(1-s), s_{\mathbb{D}}^{1} \stackrel{\text { def }}{=} \frac{R c(M-1)}{2(1-R c)}, s_{\mathbb{D}}^{2} \stackrel{\text { def }}{=} \frac{R c(M-1)}{M-R c}$. We introduce the following definitions.

Definition 2. When $q>q_{\mathbb{D}}\left(q \leq q_{\mathbb{D}}\right)$, we say that the firm is (not) sufficiently leveraged.

Definition 3. When $M<\tilde{M}(M \geq \tilde{M})$, we say that the market is (not) rapidly shrinking.

The first moniker is intuitive, since larger $q$ is tantamount to a larger debt load. Note that whether this condition holds critically depends on market parameters. In particular, firms are never sufficiently leveraged when inventory does not depreciate too rapidly (i.e., $s$ exceeds some critical value), and an important distinction is drawn depending on whether markets are "rapidly shrinking," as captured in the second definition. Since $\tilde{M}<2$, a rapidly shrinking market is reflective of a second period expected demand considerably below the first period realized demand, which is consistent with (and stronger than) our earlier condition for a "shrinking market," $M<3$.

With these definitions, we now characterize the liquidation policy of a leveraged firm.

Lemma 2 (Leveraged Firm). In equilibrium,

(a) a firm that is not sufficiently leveraged follows the first best liquidation policy, i.e., $\ell_{\mathcal{R}}(q, d)=$ $\ell^{\mathrm{fb}}(q, d)$.

(b) a firm that is sufficiently leveraged and operates in a market that is not rapidly shrinking follows a threshold liquidation policy: $\ell_{\mathcal{R}}(q, d)=\mathbb{1}\left\{d<d_{\mathcal{R}}(q)\right\}$.

(c) a firm that is sufficiently leveraged and operates in a market that is rapidly shrinking follows a non-threshold policy: $\ell_{\mathcal{R}}(q, d)=\mathbb{1}\left\{d \in\left[0, \frac{R w}{M}\right) \cup\left(d_{\mathcal{R}}(q), d^{\mathrm{fb}}(q)\right)\right\}$,

where $d_{\mathcal{R}}(q) \stackrel{\text { def }}{=} \begin{cases}\frac{R w}{M}, & \text { if } M=\tilde{M}, \\ \max \left(\frac{2 s q-R w}{M-2(1-s)}, \frac{R w}{M}\right) & \text { otherwise. }\end{cases}$

It is worth noting that a leveraged firm follows the same policy in both the upper and the lower node of subgame $\mathbf{S}$, i.e., its policy is unaffected by a covenant breach. This is intuitive, since its 
own policy becomes irrelevant when the breach happens, so that the optimal policy in equilibrium is the same as when the covenant is not breached.

Lemma 2 is summarized in Figure 5. Note that a firm's behavior critically depends on whether it is sufficiently leveraged, and on whether the market is rapidly shrinking or not. As intuition would dictate, under small enough debt levels, a leveraged firm acts as if the entire order were funded using its own equity (see Figure 5(a)). However, as leverage increases, the policy starts to deviate substantially.

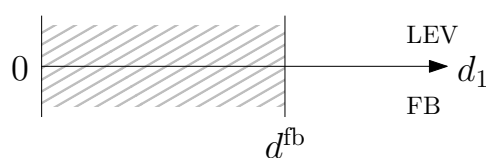

(a)

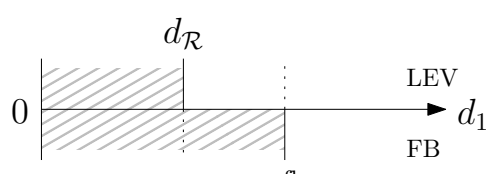

$d^{\mathrm{fb}}$

(b)

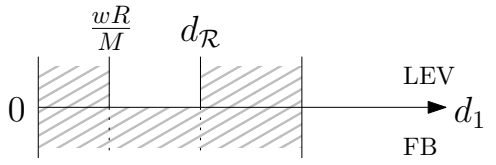

$d^{\mathrm{fb}}$

(c)

Figure 5: Firm's optimal liquidation policy as a function of realized demand $d_{1}$. The shaded area denotes a preference for liquidation at $t=1$. The policy above the horizontal axis corresponds to a leveraged firm, when it is (a) not sufficiently leveraged, or sufficiently leveraged in a market that is (b) not rapidly shrinking or (c) rapidly shrinking. For comparison, the first best policy is depicted below the axis.

When the market is not rapidly shrinking, a sufficiently leveraged firm still follows a threshold policy, but starts liquidating less often than first best, see Figure 5(b). In fact, the new threshold $d_{\mathcal{R}}(q)$ is not only lower than $d^{\mathrm{fb}}(q)$, but also increases in $q$ at a lower rate, implying that the discrepancy in policies becomes even more pronounced as leverage increases.

Surprisingly, a sufficiently leveraged firm operating in a rapidly shrinking market entirely departs from threshold policies, see Figure 5(c). To understand this behavior, note that market conditions in this case are particularly dire, as bleak second period prospects are compounded by a rapidly depreciating inventory value $\left(s<s_{\mathbb{D}}^{2}\right)$. At intermediate sales levels $\left(\frac{w R}{M}<d<d_{\mathcal{R}}(q)\right)$, the latter effect takes precedence, as liquidating (a relatively large) inventory would mean immediate insolvency or extremely low profits for a leveraged firm, while continuing could yield hope of high(er) profit if the high demand scenario materializes. As before, it is important to note that a leveraged firm liquidates less often than first best. However, the disagreement here occurs at intermediate sales levels, where the first best policy liquidates so as to recover a higher total asset value, while a leveraged firm gambles on continuation. We note that non-threshold policies are not a by-product of our assumptions, and they persist under considerably more general settings - see the discussion in Section C.1 of the Appendix.

The critical threshold $q_{\mathbb{D}}$ can also be connected with the existence of bankruptcy risk in the debt agreement, as summarized in the following result.

Lemma 3 (Sufficient leverage and bankruptcy). Under either the first best or the leveraged firm's liquidation policy, $q>q_{\mathbb{D}}$ is 
(i) a necessary and sufficient condition for bankruptcy risk in non-shrinking markets, and

(ii) a sufficient (but not necessary) condition for bankruptcy risk in shrinking markets.

The result implies that sufficiently leveraged firms always induce bankruptcy risk, and, in fact, the former phenomenon is actually synonymous with the latter in non-shrinking markets. Since such markets may be quite natural in practice, this fact bears very relevant implications for our analysis, suggesting that risky lending agreements of the type examined here are likely to involve sufficiently leveraged firms, whose liquidation policies depart from first best. Interestingly, this is not necessarily the case in shrinking markets. Note that the distinction between the two regimes is given by our earlier definition of shrinking markets (i.e., $M \gtrless 3$ ), instead of rapidly shrinking markets (i.e., $M \gtrless \tilde{M}$ ).

\subsection{Bank}

We next analyze the bank's optimal liquidation policy in subgame $\mathbf{S}$ (see Figure 4(b)).

Lemma 4 (Bank's liquidation policy). In equilibrium, the bank follows the threshold liquidation policy $\ell_{\mathcal{B}}\left(q, d_{1}\right)=\mathbb{1}\left\{d_{1}<d_{\mathcal{B}}(q)\right\}$, unless the collateral value depreciates very rapidly $\left(s<s_{\mathcal{B}}\right)$ and the firm is highly leveraged $\left(q>q_{\mathcal{B}}\right)$, in which case she follows the non-threshold policy $\ell_{\mathcal{B}}\left(q, d_{1}\right)=$ $\mathbb{1}\left\{d_{1} \in\left[0, d^{\mathrm{fb}}(q)\right) \cup\left(\frac{R w-2 q s}{1-2 s}, d_{\mathcal{B}}(q)\right)\right\}$, where $q_{\mathcal{B}} \stackrel{\text { def }}{=} \frac{R(M-1+2 s) x_{0}}{c R(M-1+2 s)-2 M s}, s_{\mathcal{B}} \stackrel{\text { def }}{=} \frac{(M-1) c R}{2(M-c R)}$, and $d_{\mathcal{B}}(q) \stackrel{\text { def }}{=}$ $R w$.

The result suggests that, barring a particular case, the bank follows a threshold liquidation policy, with a threshold $d_{\mathcal{B}}(q)$ that increases in $q, R$ and $c$, and decreases in $x_{0}$. It is important to note that the bank's own liquidation preferences are also not efficient in general. In particular, it can be checked that $\ell_{\mathcal{B}}\left(q, d_{1}\right) \geq \ell^{\mathrm{fb}}\left(q, d_{1}\right)$, reflecting the bank's preference for conservative actions that result in more liquidation than first best.

Interestingly, the bank also departs from a threshold policy, under similar conditions as the firm, i.e., low liquidation value and high leverage. Here, when the realized sales are low $\left(d_{1}<\frac{R w-2 q s}{1-2 s}\right)$, it can be checked that the firm is bankrupt, and the bank is set to seize all its assets, either at $t=1$ or at $t=2$. As such, the bank effectively becomes the owner and operator of the inventory, and prefers to follow the first best policy, liquidating below the threshold $d^{\mathrm{fb}}(q)$, and continuing otherwise.

While in this paper we assumed perfect and symmetric information, it is nonetheless interesting to briefly consider a setting where the bank is unable to directly observe the firm's first period sales. In such a case, when optimal, the bank's non-threshold liquidation policy might induce a firm with sales above $\frac{R w-2 q s}{1-2 s}$, but below $d_{\mathcal{B}}$, to underreport the sales, so as to avoid liquidation. ${ }^{9}$

\footnotetext{
${ }^{9}$ It can be checked that such a firm would prefer continuation to liquidation, and may thus choose to report sales strictly below $\frac{R w-2 q s}{1-2 s}$, but above $d^{\mathrm{fb}}$.
} 
This effect has already been documented in the context of debt-service renegotiation, where a borrower in default may overstate their debt service abatement in order to obtain better terms from the lender. Specifically, Bourgeon and Dionne (2013) find that asymmetric information about liquidation value might induce firms with high values to act as firms with lower values. In our case, sales underreporting would not be driven only by information asymmetry, but also by the players' operational preferences. Similar to Bourgeon and Dionne (2013), our model also suggests that this behavior would be more likely in markets where liquidation values are low and leverage is high.

\subsection{Liquidation Conflict}

To understand how tension between the players may arise, we now compare their optimal liquidation policies, and identify the circumstances under which they are in (dis)agreement. To this end, note that the firm would never prefer liquidating its inventory if this action lead to insolvency. As such, whenever the firm prefers liquidation, the bank is always made whole, and the two players are in agreement. When the firm prefers to continue, however, it is possible that the bank might prefer liquidation. This prompts us to introduce the following definition.

Definition 4. We define the disagreement region $\mathbb{D}$ as the set of first period demand realizations for which the liquidation preferences of the two players are misaligned. More formally,

$\mathbb{D} \stackrel{\text { def }}{=}\left\{d \geq 0 \mid X_{\mathcal{R}, \mathscr{L}}(q, d)<\mathbb{E}\left[X_{\mathcal{R}, \mathscr{C}}\left(q, d, D_{2}\right) \mid D_{1}=d\right]\right.$ and $\left.X_{\mathcal{B}, \mathscr{L}}(q, d)>\mathbb{E}\left[X_{\mathcal{B}, \mathscr{C}}\left(q, d, D_{2}\right) \mid D_{1}=d\right]\right\}$.

Whenever $\mathbb{D} \neq \emptyset$, we say that liquidation conflict exists between the two players.

Liquidation conflict here is a direct manifestation of agency issues,${ }^{10}$ driven by the shareholderdebtholder conflict of interest (Jensen and Meckling 1976, Myers 1977, Smith and Warner 1979). Intuitively, by continuing, the firm (shareholder) has limited downside and potentially large upside, due to its leverage. It is thus effectively shifting risk to the bank (debtholder). On the contrary, the bank may prefer liquidation, so as not to expose the collateral to further potential depreciation. Note that the existence and the extent of liquidation conflict critically depend on the firm's order quantity $q$ and market parameters. To this end, our next result precisely characterizes the disagreement region, showing that it is always a (possibly empty) interval of demand values, intrinsically related to whether a firm is sufficiently leveraged.

Lemma 5 (Liquidation conflict). In equilibrium, liquidation conflict arises if and only if the firm

\footnotetext{
${ }^{10}$ We note that agency issues may exist between the two players in other forms, as well, e.g., concerning the choice of initial order quantity $q$ (see, e.g., Buzacott and Zhang 2004). We use the term "liquidation conflict" to completely isolate the effect, and pinpoint that it is related to dynamic inventory decisions, i.e., liquidation policies.
} 
is sufficiently leveraged. More precisely,

$$
\begin{aligned}
& \mathbb{D}=\left\{\begin{array}{ll}
\emptyset, & \text { if } q \leq q_{\mathbb{D}}, \\
(\underline{d}(q), \bar{d}(q)), & \text { otherwise, }
\end{array}\right. \text { where } \\
& \underline{d}(q) \stackrel{\text { def }}{=}\left\{\begin{array} { l l } 
{ \operatorname { m a x } ( d _ { \mathcal { R } } ( q ) , \frac { R w - 2 s q } { 1 - 2 s } ) } & { \text { if } M \geq \tilde { M } , } \\
{ \operatorname { m a x } ( \frac { R w } { M } , \frac { R w - 2 s q } { 1 - 2 s } ) } & { \text { otherwise, } }
\end{array} \quad \text { and } \overline { d } ( q ) \stackrel { \text { def } } { = } \left\{\begin{array}{ll}
d_{\mathcal{B}}(q) & \text { if } M \geq \tilde{M}, \\
\min \left(d_{\mathcal{B}}(q), d_{\mathcal{R}}(q)\right) & \text { otherwise. }
\end{array}\right.\right.
\end{aligned}
$$

Moreover, $\bar{d}(q)-\underline{d}(q)$ is increasing in $q$.

The result shows that the two players are in complete agreement, i.e., $\mathbb{D}=\emptyset$, when the firm is not sufficiently leveraged. Otherwise, liquidation conflict always arises, at intermediate levels of sales, $(\underline{d}(q), \bar{d}(q))$. This is quite intuitive, since for low (high) enough sales, both players agree that the optimal action is to liquidate (continue). Furthermore, there is increasing conflict as leverage increases, and, ceteris paribus, conflict is more likely as the market strength $M$, the interest rate $R$ or the per-unit cost $c$ increase, or as the firm's initial capital $x_{0}$ decreases.

In view of our earlier results, liquidation conflict arises exactly when leveraged firms deviate from first best. More interestingly, note that while liquidation conflict always arises in the presence of bankruptcy risk in non-shrinking markets, that is not necessarily the case if the market is shrinking: strictly higher leverage may be required to generate liquidation conflict, than to result in bankruptcy risk (see Lemma 3).

\section{$5 \quad$ Flexibility-driven Agency Costs and Covenant Effectiveness}

The liquidation conflict identified above could potentially give rise to agency costs. To formalize this, consider a firm desiring to follow the first best actions, and thus order $q^{\mathrm{fb}}$. Since a debt contract signed at $t=0$ cannot explicitly bind the firm to follow a particular operational policy at $t=1,{ }^{11}$ once the debt is in place, the (now leveraged) firm would actually follow the liquidation policy $\ell_{\mathcal{R}}$ in equilibrium, which generally differs from the first best policy $\ell^{\mathrm{fb}}$, as discussed in Lemma 2. By rationally anticipating this risk shifting behavior at $t=1$, the bank would charge a higher interest rate, generating financing costs that could be large enough to induce the firm to reduce its initial debt burden and order quantity. This could lead to a value loss, with the firm's expected profits being lower than the maximum possible value of $V^{\mathrm{fb}}$.

Liquidation conflict and its associated agency costs shed light on why covenants may be useful. When there is disagreement concerning the liquidation decision, an appropriately crafted covenant

\footnotetext{
${ }^{11}$ This is consistent with typical assumptions in the literature on incomplete contracts (see, e.g., Aghion and Bolton 1992, Hart and Moore 1998), as well as with observed practice. Contracts that seek to prescribe actions or payments for every possible contingency would be overly complex, and would also not be enforceable ex-post in a court (Gârleanu and Zwiebel 2009, Hilson 2013, Tirole 2006).
} 
would offer the lender protection through the transfer of control rights. To see this, note that when the firm's order quantity $q$ results in liquidation conflict, i.e., $\mathbb{D} \neq \emptyset$, the inclusion of a financial covenant with a cash flow threshold $\delta \in \mathbb{D}$ would give the bank the right to force liquidation at $t=1$ whenever $d_{1} \in(\underline{d}(q), \delta)$, an action that would be optimal for her, but not for the firm. This added protection would enable the bank to lower the interest rate, and thus the agency costs of debt could be reduced. Would overly tight covenants, warranting ever decreasing interest rates, then fully alleviate agency costs? The answer is clearly no, as in that case the bank's liquidation preferences would be enforced, which we have also argued to be inefficient (Section 4.3).

This leads to the central question addressed in our paper: How effective are simple covenants in mitigating agency costs, i.e., how much value can they restore?

Theoretically, state-contingent control transfer mechanisms such as covenants could conceivably fully alleviate agency costs as long as they ensure that, in any state of the world, control rights lie with a player who prefers to follow the first best action in that particular state. In our setting, it is entirely unclear whether such a mechanism is possible, particularly when limiting attention to the simple (financial or borrowing base) covenants encountered in practice. Worse, the players' non-threshold policies might further undermine the effectiveness of such simple covenants.

To study these issues, we now formally define the agency costs of debt. To simplify contract notation, we omit the interest rate $R$, as it is always determined endogenously as a function of all other parameters and decisions, so that the bank breaks even.

For a contract $\kappa$, let $V_{\mathcal{R}}(\kappa)$ denote the maximum expected profit achievable by the firm, i.e.,

$$
\begin{array}{lll}
V_{\mathcal{R}}(\kappa) \stackrel{\text { def }}{=} & \text { maximize } & \pi_{\mathcal{R}}(q, \kappa) \\
\text { subject to } & q \geq 0 \\
& \pi_{\mathcal{B}}(q, \kappa)=0 .
\end{array}
$$

The associated agency costs correspond to the relative value loss compared with the optimal extracted value under the first best actions, i.e.,

$$
A(\kappa) \stackrel{\text { def }}{=} \frac{V^{\mathrm{fb}}-V_{\mathcal{R}}(\kappa)}{V^{\mathrm{fb}}}
$$

In equilibrium, when contracts are optimally chosen from a particular set $\mathbb{K} \in\left\{\mathbb{K}^{\emptyset}, \mathbb{K}^{\mathrm{F}}, \mathbb{K}^{\mathrm{B}}\right\}$, the resulting agency costs are given by $\inf _{\kappa \in \mathbb{K}} A(\kappa)$. The lower the equilibrium agency costs are, the more effective the set of contracts $\mathbb{K}$ is in alleviating agency issues.

In the remainder of the analysis, let $q^{\star}$ and $\ell^{\star}$ denote the equilibrium quantity and liquidation policy, respectively. 


\subsection{Interest-Rate-Only Contracts}

The following result confirms that interest-rate-only contracts generally fail to alleviate agency issues, and lead to an equilibrium where firms with low capital under-order relative to first best.

Theorem 1. Under interest-rate-only contracts,

(i) if $x_{0} \geq \tilde{x}_{0}$, $\inf _{\kappa \in \mathbb{K}^{\emptyset}} A(\kappa)=0$. In particular, there are no agency costs, $q^{\star}=q^{\mathrm{fb}}$, and $\ell^{\star}=\ell^{\mathrm{fb}}$.

(ii) if $x_{0}<\tilde{x}_{0}, \inf _{\kappa \in \mathbb{K}^{\emptyset}} A(\kappa)>0$. In particular, agency costs persist, $q^{\star}<q^{\mathrm{fb}}$, and $\ell^{\star}=\ell_{\mathcal{R}} \leq \ell^{\mathrm{fb}}$.

Here, $\tilde{x}_{0}$ is a threshold that is strictly lower than $c q^{\mathrm{fb}}$ and depends only on the market parameters $M, c, s$, and $F_{1}$ (for an explicit characterization, see the proof of the theorem). The result confirms that, under interest-rate-only contracts, firms with low initial capital will always be faced with agency costs, as their high leverage coupled with the inability to relinquish control rights will always make them "too risky" for the lender. Put differently, such firms will always be unable to harness the full benefits afforded by the flexibility of an intermediate liquidation option, because lenders will fear that this flexibility could be used to shift risk.

\subsection{Contracts With Covenants}

Surprisingly, despite the complexity of the setting, a contract with a financial covenant is able to completely alleviate agency costs and ensure that first best actions are always followed, in equilibrium.

Theorem 2. Under contracts that include financial covenants,

$$
\inf _{\kappa \in \mathbb{K}^{\mathrm{F}}} A(\kappa)=0, \forall x_{0} \geq 0
$$

In particular, there are no agency costs, and $q^{\star}=q^{\mathrm{fb}}, \ell^{\star}=\ell^{\mathrm{fb}}$.

This result critically highlights the effectiveness of simple financial covenants at dealing with agency issues that arise from dynamic inventory liquidation decisions. Financial covenants enable firms to maximally exploit the potential of a business opportunity, irrespective of their initial capital. In conjunction with Theorem 1, this result also implies that covenants are particularly effective for firms with limited initial capital; this is in line with the empirical results in Bradley and Roberts (2004), who find firms that are smaller or have fewer tangible assets to face more covenants in their debt agreements.

A direct corollary of Theorem 2 is that borrowing base covenants, which subsume financial covenants, $\mathbb{K}^{\mathrm{B}} \supset \mathbb{K}^{\mathrm{F}}$, also fully alleviate agency costs. More generally, covenants tied to the firm's cash flow appear to adequately reflect the operating flexibility provided by a liquidation option, and are thus able to fully restore firm value. In the face of additional operating flexibility, e.g., through partial liquidations or replenishments (see Section 6), this will no longer be true. 


\subsection{Optimal Covenant Design and Bankruptcy Risk}

Our analysis also allows characterizing the optimal covenant term in equilibrium, highlighting its dependence on market parameters.

Theorem 3. In equilibrium, the covenant cash flow threshold is given by $\delta^{\star}=\frac{2 s q^{\mathrm{fb}}}{M+2 s-1}$. Moreover, $\delta^{\star}$ is increasing in $s$ and $D_{1}$ in the usual stochastic order, and decreasing in $c$.

The comparative statics in Theorem 3 might initially seem surprising. Ceteris paribus, one might expect markets with lower inventory depreciation rates (i.e., larger $s$ ), lower production costs (i.e., lower $c$ ) or stronger demand (i.e., higher $D_{1}$ ) to be more secure, and to thus warrant less protection, in the form of less tight covenants. However, these conditions also lead to larger first best order quantities, and hence larger debt levels and increased "risk." We note that $\delta^{\star}$ has a nonmonotonic behavior in $M$. Intuitively, higher $M$ also induces a larger debt through a larger order, but this is primarily due to an increased second period cash flow. As such, it is unclear whether increasing the first period required cash flow, i.e., $\delta^{\star}$, would result in better risk protection. ${ }^{12}$

Finally, our analysis enables the characterization of precise conditions under which covenants are necessary, connecting these with bankruptcy risk and the firm's initial capital.

Theorem 4. In equilibrium, a covenant is necessarily included if and only if the firm's initial capital $x_{0}$ is below the threshold $\tilde{x}_{0}$. Furthermore, bankruptcy risk is:

(i) a necessary and sufficient condition for covenants to be included in a non-shrinking market,

(ii) a necessary (but not sufficient) condition for covenants to be included in a shrinking market.

Covenants are tantamount to bankruptcy risk in non-shrinking markets. Given that, in practice, bankruptcy risk persists in a vast majority of debt agreements, this suggests that covenants should also be ubiquitous. This is consistent with empirical findings: Bradley and Roberts (2004) find a positive relation between the inclusion of covenants and bankruptcy risk (as measured through credit spreads), and Roberts and Sufi (2009b) find that $97 \%$ of all loans contain at least one financial covenant. It is also aligned with insights in the finance literature, which often informally equate ${ }^{13}$ the presence of covenants to bankruptcy risk (Myers 1977). In addition, our result also highlights a distinction between bankruptcy and covenants in shrinking markets, where risky debt agreements without covenants may be possible. To the best of our knowledge, this insight is new, and is afforded exclusively by the more detailed operational model.

\footnotetext{
${ }^{12}$ It can be shown that $\delta^{\star}$ is decreasing in $M$ when $D_{1}$ is uniformly distributed. Our numerical simulations also suggest that this is the case for a Gaussian or exponential distribution.

${ }^{13}$ For instance, in a summary of his insights, Myers (1977) states that "[...] a firm with risky debt outstanding, and which acts in its stockholders' interest, will follow a different decision rule than one which can issue risk-free debt or which issues no debt at all."
} 


\section{Additional Flexibility: Partial Liquidation and Replenishment}

We investigate the impact of additional inventory management flexibility on the effectiveness and design of covenants, and on the players' equilibrium actions. We study the following two extensions.

First, the model we considered so far allowed the two players a choice between continuation and full liquidation at $t=1$. In practice, operators may have additional flexibility, and be able to conduct partial liquidations, e.g., by performing temporary promotional or clearance sales (Talluri and Van Ryzin 2004), or by closing down only a number of underperforming stores. Similarly, upon a covenant violation and the transfer of control rights, lenders may force firms to only partially liquidate, thereby increasing cash holdings and being able to continue with a leaner business.

Second, our model offered a single opportunity of ordering inventory, at $t=0$. In practical settings where lead times are not excessively long compared to the selling season, operators may have the flexibility to respond to strong sales by replenishing their stock.

We capture these realistic features by gradually enhancing the operator's flexibility, first allowing partial liquidation (Section 6.1) and then adding a replenishment option (Section 6.2). We further enrich the model by extending the two-point distribution for the second period demand to a general distribution, while preserving the correlation structure assumed thus far. Specifically, we relax Assumptions 1 and 2, replacing them with

Assumption 3. The random variable $Z$ that drives second period demand has non-negative support, with c.d.f. $F_{Z}$ and p.d.f. $f_{Z}$.

We also implicitly assume second period demand to be unaffected by the firm's inventory adjustment, ${ }^{14}$ and retain all other assumptions and notation employed so far.

\subsection{Partial Liquidation}

When partial liquidation is possible, the main changes in the game between the two players occur in the subgame $\mathbf{S}$ at the intermediate period. All definitions, such as first best actions and agency costs of debt, extend naturally to this setting. For conciseness, we describe only the sequence of actions and the outcome in the new subgame $\mathbf{S}$.

At $t=1$, the firm moves first, by observing the first period demand $d_{1}$, and deciding what fraction $\ell \in[0,1]$ of the leftover inventory to liquidate. For example, under the firm's action, $\ell \cdot\left(q-d_{1}\right)^{+}$units would be liquidated, resulting in an additional cash flow of $s \cdot \ell \cdot\left(q-d_{1}\right)^{+}$, and a starting inventory level of $(1-\ell) \cdot\left(q-d_{1}\right)^{+}$for the second selling period.

In case the loan contract includes a covenant, the bank moves second, and evaluates the covenant terms using the firm's cash flow $\min \left(q, d_{1}\right)+s \cdot \ell \cdot\left(q-d_{1}\right)^{+}$and remaining inventory $(1-\ell) \cdot\left(q-d_{1}\right)^{+}$

\footnotetext{
${ }^{14}$ For instance, this assumption would apply when liquidating geographically segregated stores or units of the same business, or returning products to the manufacturer at less than the wholesale price. For other examples, see Talluri and Van Ryzin (2004).
} 
after the firm's actions. Upon a covenant breach, the bank gains control, and decides whether and how much additional inventory should be liquidated. The outcome of the subgame is the total fraction of inventory that was liquidated as a result of actions by both players.

We start our analysis with a comparison of the player's liquidation preferences that highlights the resulting agency conflict. Let $\ell_{\mathcal{P}}: \mathbb{R} \times \mathbb{R} \rightarrow[0,1]$ denote the optimal standalone policy for player $\mathcal{P} \in\{\mathcal{R}, \mathcal{B}\}$, in case the player were solely responsible for taking liquidation actions.

Lemma 6 (Standalone liquidation policies). The players' optimal standalone policies at $t=1$, in case they were solely responsible for the liquidation actions, are such that $\ell_{\mathcal{B}}(q, d) \geq \ell_{\mathcal{R}}(q, d)$ for all $q$ and $d$. Agency conflict arises, i.e., $\ell_{\mathcal{B}}(q, d)>\ell_{\mathcal{R}}(q, d)$, if $d<d_{\mathrm{S}} \stackrel{\text { def }}{=} \frac{R w-s q}{1-s}$.

Comparing the players' standalone policies yields similar qualitative insights as our earlier analysis. In particular, the firm prefers to carry a higher stock into the second period - shifting risk - while the bank prefers lower stock so as not to expose the collateral to further potential depreciation. Conflict arises under poor first period demand, which results in large leftover inventory. In fact, when $d_{1}<d_{\mathrm{S}}$, the firm is insolvent at $t=1$, i.e., the cash flow $d_{1}+s\left(q-d_{1}\right)$ obtained by liquidating all remaining inventory would be lower than its liabilities $R w$.

Critically different from our earlier setup, however, is the fact that - in anticipation of the additional liquidation forced by the bank - the firm might now use its operating flexibility to affect and possibly avert a covenant violation, by boosting its cash flow at $t=1$ through its liquidation actions. Let $\ell_{\mathcal{R}}^{\star}$ denote the firm's equilibrium liquidation policy.

Lemma 7. In equilibrium, a financial covenant is never violated if the firm is solvent at $t=1$. More precisely, $\ell_{\mathcal{R}}^{\star}(q, d) \geq \ell_{\mathrm{C}}(q, d) \forall d \geq d_{\mathrm{S}}$, where $\ell_{\mathrm{C}}(q, d) \stackrel{\text { def }}{=} \min \left\{z \geq 0 \mid d+s(q-d)^{+} z \geq \delta\right\}$ is the minimum required liquidation so that the firm's cash flow at $t=1$ exactly covers the covenant. The result has two key implications. First, it confirms that, in equilibrium, a leveraged firm that is solvent but is faced with a covenant violation would always liquidate enough inventory to cover the covenant, allowing it to retain full control rights. This complements the debt-covenant hypothesis developed in the empirical accounting literature - which argues that managers pull accounting levers to avoid a costly covenant breach - by showing how managers can also pull operational levers to achieve the same result. This provides an alternative explanation for the empirical findings in Dichev and Skinner (2002), who document that an unusually large number of firms have financial metrics that are right at the covenant breach level. Furthermore, the policy predicted by our model is surprisingly well aligned with the actions taken by L.A. Gear's management in the aftermath of its sale to Trefoil Capital Investment L.P., as alluded to in the quote in the Introduction (see DeAngelo et al. 2002 for more details). The result also confirms and extends the intuition in Besbes and Maglaras (2012), who study non-leveraged and non-strategic firms faced with (exogenous) financial milestones, by arguing that, when feasible, it is optimal for leveraged, strategic firms to conduct fire sales in order to exactly meet a financial covenant. 
The second key implication of Lemma 7 is that financial covenants may not be effective in such settings. In particular, when the firm liquidates exactly so as to avert a covenant violation, less inventory is typically liquidated than what the lender would have preferred. Thus, agency conflict persists, and financial covenants are no longer able to restore full firm value for sufficiently leveraged firms, as formalized in the next result.

Theorem 5. Under contracts that include financial covenants,

$$
\inf _{\kappa \in \mathbb{K}^{\mathrm{F}}} A(\kappa)>0, \text { if } x_{0}<\bar{x}_{0}
$$

The threshold $\bar{x}_{0}$ depends on market parameters $M, c, s, F_{1}$ and $F_{Z}$ - see the proof of the Theorem for a characterization. To provide more intuition for their failure, note that financial covenants, by exclusively relying on cash flow, are agnostic to the firm's liquidation actions, and thus cannot distinguish "healthy sales" at full price from "fire sales." This suggests that covenants with additional "degrees of freedom" - reflective of the firm's own increased operating flexibility - are needed. To this end, we next assess the effectiveness of borrowing base covenants.

Theorem 6. Under contracts that include borrowing base covenants,

$$
\inf _{\kappa \in \mathbb{K}^{\mathrm{B}}} A(\kappa)=0, \forall x_{0} \geq 0
$$

The equilibrium borrowing base covenant sets an inventory advance rate of $\alpha^{\star}=1-\frac{2(1-s)}{s(M-1) F_{Z}^{-1}(1-s)}$, and a threshold $\beta^{\star}=s q^{\mathrm{fb}} .{ }^{15}$ Moreover, $\alpha^{\star}$ is increasing in $M$ and $Z$ in the usual stochastic order; $\beta^{\star}$ is increasing in $s$, decreasing in $c$, increasing in $M$, and increasing in $D_{1}$ and $Z$ in the usual stochastic order.

This result bears two important implications. First, it highlights the effectiveness of borrowing base covenants when dealing with inventory-heavy firms. By applying a suitable advance rate to the remaining inventory and optimally setting the minimum required borrowing base level, such covenants are able to fully mitigate the agency conflict generated by the additional operating flexibility and completely restore firm value. As an aside, since borrowing base covenants are primarily used in secured loans (Hilson 2013, Roberts and Sufi 2009a), this result also provides additional justification for inventory-heavy firms to collateralize their assets.

Second, our result suggests that advance rates against inventory collateral are critical contract terms, which should be set to properly reflect future risk: the comparative statics of $\alpha^{\star}$ suggest that stronger residual demand warrants less aggressive "hair cuts." 16 The comparative statics concerning

\footnotetext{
${ }^{15}$ As a technical remark, if $1-\frac{2(1-s)}{s(M-1) F_{Z}^{-1}(1-s)}<0$, then $\alpha^{\star}=0$ and $\beta^{\star}=s q^{\mathrm{fb}}-s\left(1-\frac{2(1-s)}{s(M-1) F_{Z}^{-1}(1-s)}\right)\left(q^{\mathrm{fb}}-d_{1}\right)$.

${ }^{16}$ It can be shown that $\alpha^{\star}$ is increasing in $s$ when $Z$ is uniformly distributed. Our numerical simulations also suggest that this is the case for a Gaussian or exponential distribution.
} 
$\beta^{\star}$ are consistent with our earlier findings in Section 5.3, confirming that lenders enforce tighter covenants in the face of more secure markets.

Together with Theorem 5, these findings provide a theoretical rationale for several practices and informal beliefs of banks lending against inventory. While borrowing base covenants and their associated advance rates are routinely used in that context, ${ }^{17}$ typical contracts often include fewer financial covenants, reflective of the practitioners' view concerning their questionable effectiveness:

"[...] facilities are typically underwritten with a limited number of financial covenants; the additional risk this poses to the bank is mitigated by conservative advance rates against liquid collateral, strong collateral controls, and frequent monitoring.[...] Some banks institute financial covenants to monitor retail borrowers, but the usefulness of financial covenants is debatable, given (1) the overwhelming reliance on collateral liquidity to repay the debt and (2) a firm's tendency to experience seasonal losses. Excess availability covenants, however, can help ensure an adequate collateral cushion in the event of liquidation and provide current and meaningful measures of liquidity; [...]" (Comptroller of the Currency, U.S. Treasury Department CH 2014)

Our results are also consistent with the empirical findings in Flannery and Wang (2011), who document that secured loans that include borrowing base covenants involve fewer financial covenants, and are particularly pertinent for smaller or riskier firms. This is the only paper we are aware of in the empirical finance literature that examines borrowing-based lines of credit.

\subsection{Partial Liquidation and Replenishment}

We now consider the case in which both partial liquidation and replenishment are possible. That is, the firm now also has the ability to place an additional order at $t=1$ at per-unit cost $c$. We model two ways the firm could finance such an order: either by borrowing more money or by using cash it generated from first-period sales. To enable the latter, we relax the lockbox account's zero withdrawal limit assumption, as per term [T2], and append the following term to any of the contracts we consider:

$[\mathrm{T} 2]^{\prime}$ The firm is allowed to withdraw cash from the lockbox account at $t=1$ up to a limit $\eta$, provided it is solely used to order additional inventory that is added to the collateral base.

For simplicity, we retain our earlier notation for the various sets of contracts, e.g., $\mathbb{K}^{\mathrm{B}}$ denotes contracts with borrowing base covenants (and term [T2]' included).

In the new subgame, the firm observes the first period demand and decides whether it would prefer to perform a partial liquidation - as outlined in the previous section - or to replenish its

\footnotetext{
${ }^{17}$ Roberts and Sufi (2009a) study both collateralized and non-collateralized loans, and document that $20 \%$ of the contracts include borrowing base contingencies, with $98 \%$ of these calculated based on accounts receivable and inventory, as in our model.
} 
inventory. The bank evaluates the covenant terms (if existent) using the firm's remaining cash and inventory after its actions. If breached, the bank gains control and decides whether and by how much the inventory position should be further adjusted, either through liquidation or replenishment. In case the firm wishes to borrow more money for replenishment, the bank extends the extra funds needed and charges an interest rate so that the (new) loan remains competitively priced.

The ability to replenish inventory endows the firm with considerable flexibility to respond to favorable market conditions. However, this may also act as an additional risk shifting lever, exacerbating agency conflict with the lender due to the firm's tendency to over-order once it is leveraged (see, e.g., Buzacott and Zhang 2004). Fortunately, a contract with a borrowing base covenant and a non-zero withdrawal limit is again able to fully alleviate agency issues and restore optimality, as formalized in our next result.

Theorem 7. Under contracts that include borrowing base covenants,

$$
\inf _{\kappa \in \mathbb{K}^{\mathrm{B}}} A(\kappa)=0, \forall x_{0} \geq 0
$$

In equilibrium, the borrowing base covenant sets an inventory advance rate of $\alpha^{\star}=1-\frac{2(1-s)}{s(M-1) F_{Z}^{-1}(1-s)}$, and a threshold $\beta^{\star}=s q^{\mathrm{fb}}$. The withdrawal limit from the lockbox account is $\eta^{\star}=\phi b-c q^{\mathrm{fb}}$, where $b \stackrel{\text { def }}{=} \min \left(q, d_{1}\right)$ is the account balance, and $\phi=c\left(1+\frac{(M-1) F_{Z}^{-1}(1-c)}{2}\right)$.

The theorem reaffirms the efficiency of borrowing base covenants when dealing with inventoryheavy firms. Despite the considerably more complex setting, such a covenant - coupled with a suitable withdrawal limit - is able to complete the financial contract, and fully restore firm value. It is worth noting that the advance rate $\alpha^{\star}$ and the minimum threshold $\beta^{\star}$ are actually identical to those derived in Section 6.1, suggesting that the primary role of the covenant is to alleviate agency issues resulting from inefficient partial liquidation. In contrast, the withdrawal limit completely mitigates conflict arising from inefficient investments, by preventing the firm from over-ordering.

As a technical remark, we note that maintaining a zero withdrawal limit and relying only on borrowing more money for reordering would have achieved the same purpose here. However, reordering with generated cash subject to a withdrawal limit is also natural: it is consistent with practice, and with our earlier assumption concerning the role of the lockbox in limiting cash diversion (and the associated agency conflict). Furthermore, allowing the withdrawal limit to depend on the cash balance is also very natural. In practice, such limits are often imposed through reinvestment or incurrence-based covenants, i.e., covenants that become active only when the borrower seeks to issue new debt, and limit the new loan size depending on the pre-existing debt and cash funds (see, e.g., Nini et al. (2009) and industry notes by Goodison (2011) and Morse (2014) for more details). It is worth noting that $\eta$ does not have a precise monotonicity in any of the parameters, reflective of the ambivalent effects at play. For instance, a larger $M$ warrants larger investments (and hence 
a larger withdrawal limit), but also reflects higher bankruptcy risk due to the larger leverage.

In summary, our findings in Section 6 send a clear and concrete message to inventory-heavy firms and lenders alike. First, our results suggest that a cash-poor firm that is contemplating operational changes to enhance its flexibility (such as the ability to liquidate some of its inventory or a reduction in lead time to allow mid-season replenishments) should not hesitate to enter debtfinancing agreements of the type commonly employed in practice. When suitably designed, such agreements would allow the firm to reap the full benefits of the extra flexibility, irrespective of its actual leverage. However, the firm should also be willing to cede more control as the investments it is contemplating yield more flexibility or upside, by, e.g., collateralizing its inventory and abiding by more and/or tighter covenants. For lenders, our results confirm that secured loans may be extremely effective for inventory-heavy firms, and that financial contracts underpinning such transactions are better served by borrowing base covenants rather than (cash-flow-based) financial covenants.

\section{Implications and Limitations}

In this paper, we highlighted that a firm's flexible operating capabilities can have unintended consequences on its performance, due to the large agency costs induced under leverage through the risk shifting potential. Given the indispensable role of both flexibility and debt in driving the growth strategies of firms, we believe this is a topic of utmost importance that has received very limited attention in the operations management $(\mathrm{OM})$ literature. In particular, while OM research has developed many insights concerning the benefits of flexibility, the magnitude of the associated agency costs and the ability of common terms included in debt contracts to alleviate them are not well understood.

The goal of the present paper was to make headway in this direction. We took the perspective of an inventory-heavy firm (e.g., a firm or a manufacturer), and demonstrated that flexibility in adjusting inventory levels could result in agency costs that erase significant firm value when debt contracts only include competitively-priced interest rates. The inclusion of properly structured financial or borrowing base covenants, routinely used in practice, proved to be remarkably effective at addressing these agency issues and restoring firm value.

Our core findings give rise to a series of managerial implications and empirical predictions, which we outline next, followed by a discussion of our work's limitations.

\subsection{Managerial Implications}

Our work sheds light on a new dimension managers should consider when investing in increased flexibility. While traditional wisdom suggests that the manager should trade off the technology/infrastructure upgrade costs with the operational benefits of extra flexibility, this view ignores 
that flexibility could result in increased borrowing costs and/or reduced debt capacity. This latter component of the tradeoff calculation can be of substantial magnitude, and eventually be the decisive factor driving the firm's operating strategy.

In the context of inventory adjustment flexibility, we argue that borrowing costs should not increase in practice, as simple covenants are effective in alleviating the associated agency issues. These findings send a clear and concrete message to an inventory-heavy firm that contemplates operational changes to enhance flexibility, such as lead time reduction to allow for a mid-season replenishment or the addition of separate sales channels to allow for (partial) inventory liquidation. Such a firm should not hesitate to finance its operations through debt: by suitably structuring its debt contracts, it can reap the full benefits of the extra flexibility, irrespective of leverage.

To this end, our results show that it is in the firm's best interest to ensure that the design of debt contracts (and covenants) always adequately reflects its operating capabilities and market conditions. Specifically, for inventory-heavy firms, we find that financial covenants reflect full liquidation decisions (such as store closures or product discontinuations), while borrowing base covenants are needed for partial liquidations and/or replenishment capabilities. Furthermore, we show that covenants must be tighter under lower inventory depreciation rates, higher margins, or stronger product demand. These findings have several core implications. First, they suggest that firms with enhanced flexibility and/or operating in better markets should abide by more intense and/or tight covenants. This may seem counterintuitive to an operator, as (i) the better markets may seem more "secure," and (ii) the covenants could restrict the firm's operating flexibility. Second, they suggest that suitably designed covenants would also allow operators to focus on their core competency, i.e., running the firm's operations without concern of the capital structure.

Our work also has several implications for circumstances under which financial frictions persiste.g., due to improperly designed contracts or monopolistic lending markets-where we show that distortions may arise in both operating policies and information disclosure incentives. Specifically, we argue that both the firm's managers and its creditors may follow non-intuitive operating policies, concerning the discontinuation of product lines or store closures, that are not driven by simple sales thresholds: they may prefer continuation for weak sales, and discontinuation for strong sales. Furthermore, managers may also prefer to distort information disclosures, by under-reporting the firm's sales in order to avert creditor-imposed store closures. Finally, we also argue that monopolistic lenders use covenants so as to improve their returns and lower bankruptcy risk, exacerbating operating distortions and leading to loss in firm value.

\subsection{Empirical Predictions}

Our results lead to several predictions that can be tested empirically, which we discuss next.

Prediction 1. Inventory-heavy firms with more operating flexibility have higher leverage and issue 
more bank debt.

In the finance literature, it is known that operating flexibility can have ambivalent effects on a firm's borrowing costs, decreasing them - when the ability to adapt to market conditions is used to lower the risk of default (Mauer and Triantis 1994) - but also possibly increasing them, when lenders believe the extra flexibility is used to shift risk (Myers 1977). In this context, MacKay (2003) is the first paper to empirically examine which of the two effects is the dominant one, concluding that it is the latter, so that firms with more production flexibility generally have lower leverage, and issue less public debt. The implicit factor driving this result is that covenants or other risk shifting mitigating mechanisms employed in practice do not prove effective. Our results nuance and partially counter this prediction, while confirming more bank debt issuance. More precisely, for inventory-heavy firms, Theorems 2, 6, and 7 suggest that simple financial contracts (with covenants) can completely mitigate the risk shifting behavior, leaving only the positive aspect of extra flexibility, and thus leading to larger leverage. At the same time, these results also show that borrowing base covenants may become critical as operating flexibility increases, suggesting that such firms are more likely to raise bank debt, which allows for asset collateralization and more proactive monitoring by lenders. This also leads to the following associated prediction.

Prediction 2. For inventory-heavy firms, increased operating flexibility is positively related to (a) the presence of borrowing base covenants (and collateralization), and (b) covenant intensity.

To the best of our knowledge, these predictions have not been previously formalized, and are afforded by the more detailed operational model of an inventory-heavy firm. For a more detailed discussion and justification, we refer to Section 6.2. Our analytical results in Lemma 7 and Theorem 5 also suggest that inventory-heavy firms will use the partial inventory liquidation flexibility to avert violations of financial (cash-flow-based) covenants, leading to the following empirical prediction.

Prediction 3. Inventory-heavy firms with increased operating flexibility that are only faced with financial (cash-flow-based) covenants will tend to (a) engage in fire-sales and liquidations close to reporting periods, and (b) report covenant ratios near the trigger points.

This is aligned with the empirical findings in Dichev and Skinner (2002), who document that an unusually large number of firms have financial metrics that are right at the covenant breach level, and also with the actions documented in the empirical case study on L.A. Gear (DeAngelo et al. 2002). In a different (but related) sense, Theorems 5, 6, and 7 also suggest that for firms with increased inventory-management flexibility, financial covenants may be of limited use, but borrowing base covenants may be very effective. This is consistent with practice (CH 2014, Hilson 2013), and also with empirical findings in Flannery and Wang (2011), who document that secured lending agreements that include borrowing base covenants involve fewer financial covenants, and are particularly pertinent for smaller or riskier firms. 
Our comparative statics analysis also generates several predictions concerning the covenant tightness, and its dependency on the firm's operating characteristics and market conditions. More precisely, Theorems $1,3,5,6$, and 7 generate the following prediction.

Prediction 4. Inventory-heavy firms will be faced with tighter covenants when they have (a) fewer assets or (b) higher leverage, or when they operate in markets with (c) lower inventory depreciation rates, (d) higher margins or (e) stronger demands.

To the best of our knowledge, while the empirical literature has focused extensively on (the determinants of) covenant intensity, it has paid less attention to their tightness, and its dependence on firm/market characteristics. ${ }^{18}$ Demiroglu and James (2010) find a positive relationship between covenant tightness and (a) the riskiness of the borrowers, and (b) improvements in the covenant variable. This is consistent with our prediction that better market conditions - which are typically positively related to improvements in the covenant variable - also warrant tighter covenants. To the extent that covenant intensity is positively related to tightness, our predictions are also very well aligned with Bradley and Roberts (2004) and Billett et al. (2007), who document that firms that are smaller, have fewer tangible assets, or have greater growth opportunities face more covenants. Furthermore, Billett et al. (2007) find that the presence of covenants significantly attenuates the negative relation between leverage and growth opportunities, consistent with our results that covenants are effective in alleviating agency costs of debt for high-growth firms.

Finally, the results in Theorem 4 and 8 also lead to the following prediction.

Prediction 5. In less competitive lending markets, inventory-heavy firms face more intense covenants, and have less financial leverage.

To the best of our knowledge, this has not been previously hypothesized, as the finance literature is predominantly focused on competitive lending markets.

\subsection{Limitations and Future Directions}

In modeling flexibility-driven agency costs, we made a specific choice of operating capability, i.e., inventory liquidation and replenishment. In reality, there are several other capabilities one could model, such as sourcing from multiple suppliers, switching between different products, dynamic pricing, etc. The effectiveness of common covenants in alleviating agency issues in these alternate settings is an open question, and in our view is an interesting new line of research for the operations management community.

In the context of inventory flexibility, it may also be interesting to extend our model to an arbitrary number of periods. While our intuition is that the qualitative insights we obtained will

\footnotetext{
${ }^{18}$ The most relevant paper we are aware of that studies tightness is Demiroglu and James (2010), who state that "where covenant thresholds are established is an important but heretofore largely overlooked aspect of loan contracting."
} 
persist if the covenant monitoring points coincide with the firm's decisions points, these results may change in the absence of adequate monitoring frequency. Furthermore, a multi-period setting would allow studying other interesting questions pertaining to the firm's financial structure and its interplay with operations. For instance, while it is known that the choice of debt maturity may act as a substitute for covenants in other settings (Billett et al. 2007), it is unclear whether that would persist for inventory-heavy firms.

Lastly, our study focused on the design of contracts that fully alleviated agency costs. An interesting direction of future research is to quantify agency costs in case such contracts are not used. Preliminary numerical studies for the case where interest-rate-only (financial-covenant only) contracts are used for the flexvendor (with re-ordering) are included in Section A of the Appendix. The studies suggest that (1) flexibility-driven agency costs have a first order effect on firm value in the absence of covenants, and (2) covenants that are not fully aligned with the firm's operating flexibility are effective in mitigating agency costs, although the persisting costs are still non-trivial.

\section{Acknowledgements}

We thank the Department Editor Serguei Netessine, the Associate Editor and three anonymous referees, Vlad Babich, Gerard Cachon, Jiri Chod, Darrell Duffie, Marshall Fisher, Nicolae Gârleanu, Gustavo Manso, Ananth Raman, Michael Roberts, Berril Toktay, Alex Yang, and Jeffrey Zwiebel for their valuable input. Tsoukalas is thankful to the Fishman-Davidson Center for Service and Operations Management and to the Wharton School Dean's Research Fund for grants that supported this work.

\section{References}

Aghion, P, P Bolton. 1992. An incomplete contracts approach to financial contracting. Review of Economic Studies 59(3) 473-494.

Archibald, TW, LC Thomas, JM Betts, RB Johnston. 2002. Should start-up companies be cautious? inventory policies which maximize survival probabilities. Management Science 48(9) 1161-1174.

Babich, V, MJ Sobel. 2004. Pre-ipo operational and financial decisions. Management Science 50 935-948.

Berlin, M, L Mester. 1992. Debt covenants and renegotiation. Journal of Financial Intermediation 2 95-133.

Besbes, O, C Maglaras. 2012. Dynamic pricing with financial milestones: Feedback-form policies. Management Science 9(9) 1715-1731.

Billett, Matthew T., Tao-Hsien Dolly King, David C. Mauer. 2007. Growth opportunities and the choice of leverage, debt maturity, and covenants. The Journal of Finance 62(2) 697-730.

Birge, JR, RP Parker, SA Yang. 2015. The supply chain effects of bankruptcy. Management Science 10(61).

Birge, JR, SA Yang. 2013. How inventory is (should be) financed: trade credit in supply chains with demand uncertainty and costs of financial distress. Working Paper. 
Bolton, P. 2013. Corporate finance, incomplete contracts, and corporate control. The Journal of Law, Economics, and Organization doi:10.1093/jleo/ewt010.

Bolton, P, M Dewatripont. 2004. Contract Theory. The MIT Press.

Bourgeon, JM, G Dionne. 2013. On debt service and renegotiation when debt-holders are more strategic. $J$. Finan. Intermediation 22 353-372.

Boyabatl, O, T Leng, L B Toktay. 2015. The impact of budget constraints on flexible versus dedicated technology choice. Management Science.

Boyabatlı, O, L.B. Toktay. 2011. Stochastic capacity investment and flexible versus dedicated technology choice in imperfect capital markets. Management Science 20(6) 2163-2179.

Bradley, M., M. R. Roberts. 2004. The structure and pricing of corporate debt covenants. Working Paper.

Buzacott, AJ, QR Zhang. 2004. Inventory management with asset-based financing. Management Science 50(9) 1274-1292.

Cachon, GP, AG Kok. 2007. Implementation of the newsvendor model with clearance pricing: how to (and how not to) estimate a salvage value. Manufacturing \& Service Operations Management 9(3) 276-290.

CH. 2014. Accounts Receivable and Inventory Financing. Comptroller of the currency, administrator of national banks.

Childs, PD, DC Mauer, SH Ott. 2005. Interactions of corporate financing and investment decisions: The effects of agency conflicts. Journal of Financial Economics 76 667-90.

Chod, J. 2015. Inventory, risk shifting, and trade credit. Working Paper.

Chod, J, J Zhou. 2014. Resource flexibility and capital structure. Management Science 60(3) 708-729.

Craig, N, A Raman. 2013. Improving store liquidation. Harvard Business School Working Paper.

Dada, M, Q Hu. 2008. Financing newsvendor inventory. Operations Research Letters 36(5) 569-573.

Damodaran, A. 2015. Margins by sector (us). NYU. Available online at http://pages.stern.nyu.edu/ adamodar/New_Home_Page/datafile/margin.html.

DeAngelo, H, L DeAngelo, KH Wruck. 2002. Asset liquidity, debt covenants, and managerial discretion in financial distress: the collapse of l.a. gear. Journal of Financial Economics 64 3-34.

Demiroglu, C, CM James. 2010. The information content of bank loan covenants. Review of Financial Studies 23(10) 3700-3737.

Dichev, ID, DJ Skinner. 2002. Large-sample evidence on the debt covenant hypothesis. Journal of Accounting Research 40(4) 1091-1123.

Fisher, M, K Rajaram, A Raman. 2001. Optimizing inventory replenishment of retail fashion products. Manufacturing \&3 Service Operations Management 3(3) 230-241.

Fisher, M, A Raman. 1996. Reducing the cost of demand uncertainty through accurate response to early sales. Operations Research 44(1) 87-89.

Flannery, Mark J., Xiaohong Wang. 2011. Borrowing base revolvers: Liquidity for risky firms. Tech. rep., Warrington College of Business, University of Florida. Working paper.

Gamba, A, AJ Triantis. 2014. How effectively can debt covenants alleviate financial agency problems? Working Paper. 
Gârleanu, N, J Zwiebel. 2009. Design and renegotiation of debt contracts. RFS 22(2) 749-81.

Gaur, V, S Kesavan, A Raman. 2014. Retail inventory: Managing the canary in the coal mine. California Management Review 56(2) 55-76.

Gigler, F, C Kanodia, H Sapra, R Venugopalan. 2009. Accounting conservatism and the efficiency of debt contracts. Journal of Accounting Research 47(3) 767-797.

Gong, X, X Chao, D Simchi-Levi. 2014. Dynamic inventory control with limited capital and short-term financing. Naval Research Logistics 61(3) 184-201.

Goodison, Eric. 2011. Covenant-lite loans traits and trends. Industry Note. Available online at http: //www.paulweiss.com/media/105718/plj_sep11.pdf. Link last retrieved on May 20, 2015.

Grossman, Sanford J., Oliver D. Hart. 1986. The costs and benefits of ownership: A theory of vertical and lateral integration. Journal of Political Economy 94(4) pp. 691-719.

Hart, O, J Moore. 1998. Default and renegotiation: a dynamic model of debt. QJE 113(1) 1-41.

Hilson, JF. 2013. Asset-based lending: a practical guide to secured financing. Practicing Law Institute, NY.

Jensen, MC, WH Meckling. 1976. Theory of the firm: Managerial behavior, agency costs, and capital structure. Journal of Financial Economics 3 305-360.

Kouvelis, P. 2012. Handbook of Integrated Risk Management in Global Supply Chains. Wiley, New York.

Kouvelis, P, W Zhao. 2012. Financing the newsvendor: supplier vs. bank, and the structure of optimal trade credit contracts. Operations Research 60(3) 566-580.

Leland, HE. 1994. Corporate debt value, bond covenants, and optimal capital structure. Journal of Finance 49(4) 1213-52.

Leland, HE. 1998. Agency costs, risk management, and capital structure. Journal of Finance 53 1213-1243.

MacKay, P. 2003. Real flexibility and financial structure: An empirical aanalysis. Review of Financial Studies 16(4) 1131-1165.

Manso, G. 2008. Notes and comments on investment reversibility and agency cost of debt. Econometrica 76(2) $437-442$.

Matvos, G. 2013. Estimating the benefits of contractual incompleteness. Review of Financial Studies 26(11) 2798-2844.

Mauer, DC, AJ Triantis. 1994. Interactions of corporate financing and investment decisions: A dynamic framework. Journal of Finance XLIX(4) 1253-1277.

Mello, SA, JE Parsons. 1992. Measuring the agency cost of debt. Journal of Finance 47 1887-1904.

Morellec, E. 2001. Asset liquidity, capital structure, and secured debt. Journal of Financial Economics $6 \mathbf{1}$ $173-206$.

Morse, David. 2014. Pressure remains high on lenders. Industry Note. Available online at http://www . themiddlemarket. com/maj/2011_66/pressure-remains-high-on-lenders-247559-1.html?pg=1. Link last retrieved on May 20, 2015.

Moyen, Nathalie. 2007. How big is the debt overhang problem? Journal of Economic Dynamics and Control 31(2) 433-472.

Myers, SC. 1977. Determinants of corporate borrowing. Journal of Financial Economics 5 147-145. 
Nini, G, DC Smith, A Sufi. 2009. Creditor control rights and firm investment policy. Journal of Financial Economics 92 400-420.

Phillips, R. 2005. Pricing and revenue optimization. Stanford Business Books.

Porteus, EL. 1972. Equivalent formulations of the stochastic cash balance problem. Management Science 19(3) 250-53.

Porteus, Evan L. 2002. Foundations of Stochastic Inventory Management. Stanford Business Books.

Rapaport, L, Z Tracer. 2011. Hewlett-packard cuts touchpad prices to compete with apple. Bloomberg Article. Available online at http://www.bloomberg.com/news/articles/2011-08-12/ hewlett-packard-cuts-touchpad-prices-by-100-to-as-low-as-400.

Roberts, M. R., Amir Sufi. 2009a. Renegotiation of financial contracts: Evidence from private credit agreements. Journal of Financial Economics 93 159-184.

Roberts, MR, A Sufi. 2009b. Control rights and capital structure: An empirical investigation. The Journal of Finance 64(4) 1657-1695.

Smith, CW, JB Warner. 1979. On financial contracting: An analysis of bond covenants. Journal of Financial Economics 3 33-45.

Sridhar, SS, RP Magee. 1997. Financial contracts, opportunism, and disclosure management. Review of Accounting Studies 1 225-258.

Swinney, R, S Netessine. 2009. Long-term contracts under the threat of supplier default. Manufacturing $\mathcal{E}$ Service Operations Management 11(1) 109-127.

Talluri, K, GJ Van Ryzin. 2004. The theory and practice of revenue management. Springer.

Tirole, J. 2006. The Theory of Corporate Finance. Princeton University Press.

Titman, S, S Tompaidis, S Tsyplakov. 2004. Market imperfections, investment flexibility and default spreads. Journal of Finance LIX(1) 165-205.

Topkis, D.M. 1998. Supermodularity and Complementarity. Princeton University Press.

Wikipedia. 2011. Hp touchpad. Wikipedia article. Available online at https://en.wikipedia.org/wiki/ HP_TouchPad.

Xu, X, JR Birge. 2004. Joint production and financing decisions: Modeling and analysis. Working Paper. 


\section{Appendices}

\section{A Agency Costs Under Suboptimal Contracts: Numerical Studies}

We study cases where contracts that are unable to fully alleviate agency costs are used and numerically assess their effectiveness. We rely on the flexvendor model introduced in Section 2 and extend the numerical experiments as follows. We vary the flexvendor's unit cost $c$, the liquidation value $s$ and equity $x_{0}$. All other model parameters retain their values. Tables 3 and 4 report the agency costs (1) in the absence of a covenant, formally $\inf _{\kappa \in \mathbb{K}^{\emptyset}} A(\kappa)$, and (2) when only a financial covenant is used, formally $\inf _{\kappa \in \mathbb{K}^{\mathrm{F}}} A(\kappa)$.

The findings suggest that, in the absence of covenants, flexibility-driven agency costs average at $12.52 \%$, while they can be as high as $41.9 \%$ (see Table 4 ). The inclusion of a financial covenant reduces agency costs to an average of $2.74 \%$, while persisting costs could be as high as $13 \%$.

\begin{tabular}{ccc}
\hline & $\begin{array}{c}\text { AC without covenant } \\
\inf _{\kappa \in \mathbb{K}^{\emptyset}} A(\kappa)(\text { in } \%)\end{array}$ & $\begin{array}{c}\text { AC with financial covenant } \\
\inf _{\kappa \in \mathbb{K}^{\mathrm{F}}} A(\kappa)(\text { in } \%)\end{array}$ \\
\hline min & 0.00 & 0.00 \\
average & 12.52 & 2.74 \\
$\max$ & 41.93 & 12.99 \\
\hline
\end{tabular}

Table 3: Minimum, average and maximum agency costs (AC) across all runs from Table 4. 


\begin{tabular}{|c|c|c|c|c|}
\hline $\begin{array}{l}\text { unit cost } \\
\qquad c\end{array}$ & $\begin{array}{l}\text { liq. price } \\
s\end{array}$ & $\begin{array}{c}\text { equity } \\
x_{0}\end{array}$ & $\begin{array}{l}\text { AC without cov. } \\
\inf _{\kappa \in \mathbb{K}^{\emptyset}} A(\kappa)(\text { in } \%)\end{array}$ & $\begin{array}{l}\mathrm{AC} \text { with fin. cov } \\
\inf _{\kappa \in \mathbb{K}^{\mathrm{F}}} A(\kappa)(\text { in } \%)\end{array}$ \\
\hline 0.2 & 0.1 & 0.1 & 0.62 & 0.00 \\
\hline 0.2 & 0.1 & 0.2 & 0.49 & 0.07 \\
\hline 0.2 & 0.1 & 0.4 & 0.24 & 0.00 \\
\hline 0.2 & 0.1 & 0.8 & 0.00 & 0.00 \\
\hline 0.4 & 0.1 & 0.1 & 6.73 & 0.96 \\
\hline 0.4 & 0.1 & 0.2 & 6.20 & 0.62 \\
\hline 0.4 & 0.1 & 0.4 & 5.52 & 0.52 \\
\hline 0.4 & 0.1 & 0.8 & 3.64 & 0.38 \\
\hline 0.4 & 0.3 & 0.1 & 1.47 & 0.22 \\
\hline 0.4 & 0.3 & 0.2 & 0.71 & 0.00 \\
\hline 0.4 & 0.3 & 0.4 & 0.65 & 0.22 \\
\hline 0.4 & 0.3 & 0.8 & 0.00 & 0.00 \\
\hline 0.6 & 0.1 & 0.1 & 22.63 & 5.73 \\
\hline 0.6 & 0.1 & 0.2 & 21.15 & 4.59 \\
\hline 0.6 & 0.1 & 0.4 & 18.28 & 3.41 \\
\hline 0.6 & 0.1 & 0.8 & 13.52 & 1.93 \\
\hline 0.6 & 0.3 & 0.1 & 14.23 & 2.98 \\
\hline 0.6 & 0.3 & 0.2 & 12.70 & 2.84 \\
\hline 0.6 & 0.3 & 0.4 & 10.37 & 2.32 \\
\hline 0.6 & 0.3 & 0.8 & 6.23 & 0.58 \\
\hline 0.6 & 0.5 & 0.1 & 4.22 & 0.59 \\
\hline 0.6 & 0.5 & 0.2 & 3.42 & 1.08 \\
\hline 0.6 & 0.5 & 0.4 & 1.68 & 0.59 \\
\hline 0.6 & 0.5 & 0.8 & 0.07 & 0.00 \\
\hline 0.8 & 0.1 & 0.4 & 31.21 & 11.58 \\
\hline 0.8 & 0.1 & 0.8 & 20.51 & 8.33 \\
\hline 0.8 & 0.3 & 0.2 & 30.34 & 12.99 \\
\hline 0.8 & 0.3 & 0.4 & 24.79 & 9.09 \\
\hline 0.8 & 0.3 & 0.8 & 15.49 & 3.58 \\
\hline 0.8 & 0.5 & 0.1 & 23.11 & 6.83 \\
\hline 0.8 & 0.5 & 0.2 & 26.43 & 7.50 \\
\hline 0.8 & 0.5 & 0.4 & 20.19 & 6.84 \\
\hline 0.8 & 0.5 & 0.8 & 11.13 & 2.35 \\
\hline 0.8 & 0.7 & 0.1 & 41.93 & 0.00 \\
\hline 0.8 & 0.7 & 0.2 & 37.01 & 1.52 \\
\hline 0.8 & 0.7 & 0.4 & 23.40 & 1.14 \\
\hline 0.8 & 0.7 & 0.8 & 2.80 & 0.00 \\
\hline
\end{tabular}

Table 4: Agency costs (AC) incurred (in \%) with and without a financial covenant. 


\section{B Covenants Under Monopolistic Lending}

We re-evaluate the effectiveness of covenants when the lender operates in a monopolistic market. This may be relevant in cases where the collateral is highly specialized, and only a confined number of lenders have the adequate expertise as far as valuation, assessment and potential liquidation are concerned. Furthermore, several papers in the operations management literature have considered this alternative setting, e.g., Buzacott and Zhang (2004), Dada and Hu (2008), and Boyabatlı and Toktay (2011).

Under a monopolistic lending market, both players choose their actions to maximize their expected profits. We consider a Stackelberg game in the setting described in Section 3 (i.e., under $0 / 1$ liquidation), with the bank leading by choosing the contract $\kappa$, and the firm choosing $q$ solving (3). Let $q^{\star}(\kappa)$ be its optimal order quantity, assumed unique to avoid unnecessary technical complications. The sequence of events under this alternative game is illustrated in Figure 6 .

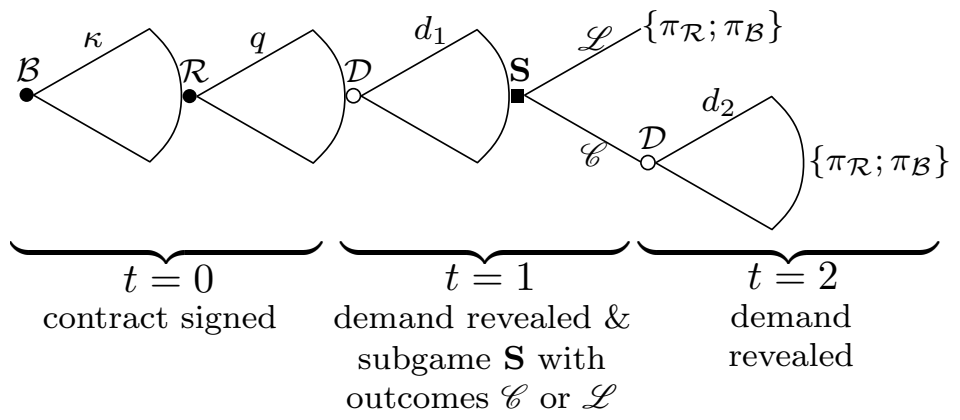

Figure 6: Game under a monopolistic lending market.

The bank's choice of contract $\kappa$ is now affected by certain trade-offs involving the firm's response. Ceteris paribus, as the bank increases the covenant tightness, her expected profit increases, while the firm's expected profit decreases. The latter fact might then induce the firm to adjust its order quantity in order to mitigate its losses, a strategic response that may reduce the bank's profit, as well. Furthermore, the interaction of the covenant with other contract terms is potentially unclear. One may speculate that the protection afforded by a covenant might be equivalent to an interest rate increase, as far the lender's expected profit is concerned. However, as highlighted in the discussion in Section 4.4, covenants allow lenders to dynamically react as new information is revealed, unlike interest rates.

The next result formalizes the conditions under which covenants persist in equilibrium under a monopolistic lending market, connecting them with bankruptcy risk.

Theorem 8. In equilibrium, ${ }^{19}$ under a monopolistic lending market, a covenant is necessarily

\footnotetext{
${ }^{19}$ We assume that $\left.\frac{\partial^{2} \pi_{\mathcal{R}}}{\partial q^{2}}\right|_{q^{\star}}<0$ holds. This technical condition is rather harmless in practice. It is only slightly stronger than requiring $q^{\star}$ to be a strict local optimum of $\pi_{\mathcal{R}}$.
} 
included if and only if there is liquidation conflict. Furthermore, in equilibrium, bankruptcy risk is (i) a sufficient condition for covenants to be included in non-shrinking markets, and

(ii) neither sufficient, nor necessary for covenants to be included in shrinking markets.

In a certain sense, the results mirror the findings under a perfectly competitive lending market. More precisely, under liquidation conflict, including covenants is always optimal for the lender, despite the strategic response of the firm, so that covenants emerge as necessary terms in lending agreements, non-substitutable by adjustments to interest rates and/or loan limits. Note that this is in contrast with the relationship between the latter two terms: it is known that, in the absence of other considerations, loan limits are substitutable by interest rates under monopolistic lending markets (see, e.g., Buzacott and Zhang 2004, Dada and Hu 2008).

However, different from the results under perfect competition, the optimal covenant in a monopolistic setting does not necessarily restore first best. This is also supported by our extensive numerical studies described below, and is not surprising (Dada and $\mathrm{Hu} 2008$ find a similar insight in a single-period newsvendor model, where lenders only optimize interest rates). Correspondingly, the optimal covenant does not alleviate operational distortions, either - in particular, the surprising non-threshold policies we found in Section 4 can persist in equilibrium (see Appendix C.2).

Interestingly, when compared with our earlier results in Theorem 4, bankruptcy is no longer a necessary condition for covenants to exist in non-shrinking markets, but is still sufficient. Loosely speaking, covenants are no longer synonymous with bankruptcy risk, but are just implied by it. The reason behind this discrepancy is that, by using covenants, a monopolist bank may be able to completely eliminate bankruptcy risk in some cases. In shrinking markets, bankruptcy risk is no longer indicative for the presence of covenants. Specifically, it is possible that contracts include convenants that in fact eliminate bankruptcy risk, as remarked above, or that there is bankruptcy risk that does not necessarily result in liquidation conflict, and thus makes covenants superfluous. It is interesting to note that Theorem 8, interpreted in a different light, also suggests that covenants would be more prevalent under monopolistic settings compared to perfect competition (after adjusting for bankruptcy risk).

To conclude this section, we note that although covenants emerge once again as critical contractual terms in debt agreements, they play a different role under a monopolistic setting, serving more as a value extraction mechanism for the lender, rather than a vehicle for restoring firm value.

\section{Robustness of Non-threshold Liquidation Policies}

In this section, we confirm that our insights and results pertaining to non-threshold policies are robust, and persist under more general demand distributions, as well as in equilibrium. 


\section{C.1 General Demand Distribution}

Our main treatment concerning the players' liquidation policies was conducted under Assumption 1, namely that the second period demand was following a discrete, two-point conditional distribution. In this context, we showed that, under debt, any of the players may find it optimal to follow a nonthreshold liquidation policy (see Lemma 2 and Lemma 4). We show that these insights continue to hold for much more general demand distributions.

Theorem 9. Suppose that, conditional on the first period demand, the second period demand distribution has a log-concave density function. Then, the firm may follow a non-threshold liquidation policy. In particular, its liquidation policy has at most two switching points, i.e., there exist (not necessarily identical) $\xi_{1}, \xi_{2} \in[0, q]$ with $\xi_{1} \leq \xi_{2}$, such that

$$
\ell_{\mathcal{R}}(q, d)=\mathbb{1}\left\{d \in\left(\xi_{1}, \xi_{2}\right)\right\}
$$

Moreover, the bank also follows a liquidation policy with at most two switching points, and the result is true for any second period demand distribution.

We note that log-concave distributions (i.e., with probability densities whose logarithm is a concave function) have been studied extensively in operations management (they are also known as Polya frequency of order 2 - see, e.g., Porteus (2002) for an overview). It is known that many of the common distributions are log-concave, for instance the exponential, the reflected exponential, the uniform, the Erlang, the normal, and all truncations, translations and convolutions of such distributions (Porteus 2002). In this sense, our assumption on the demand is not very limiting, and is well aligned with standard assumptions made in operations management.

The result confirms that two types of liquidation policies are possible. A player either follows a threshold policy, i.e., liquidating below a demand threshold and continuing above (where the threshold can also be 0 or $q$ ), or follows a non-threshold policy, preferring continuation below a threshold $\xi_{1}$ and above a threshold $\xi_{2}>\xi_{1}$, and preferring liquidation between $\xi_{1}$ and $\xi_{2}$. These are exactly the two possible patterns encountered in our base model (see Lemma 2 and Lemma 4), and, as such, our insights concerning the implication of non-threshold liquidation policies persist.

\section{C.2 Non-threshold Policies in Equilibrium}

We now confirm that, when the lender is monopolistic, non-threshold policies can persist in equilibrium, for either of the players. We provide both sufficient conditions, as well as particular instances.

Proposition 1. (a) [Firm] Suppose that $s<\frac{c(M-1)}{M-c}, M<\tilde{M}$, and $D_{1} \geq \frac{x_{0}}{c-\frac{s M}{M+s-1}}>0$ almost surely. Then, the firm always follows a non-threshold liquidation policy in equilibrium. One such instance is given by $s=\frac{1}{16}, c=\frac{1}{4}, x_{0}=1$ and $M=\frac{3}{2}$. 
(b) [Bank] Suppose that $s<\frac{(M-1) c}{2(M-c)}$ and $D_{1} \geq \frac{(M-1+2 s) x_{0}}{c(M-1+2 s)-2 M s}>0$ almost surely. Then, the bank always follows a non-threshold liquidation policy in equilibrium. One such instance is given by $s=\frac{1}{16}, c=\frac{1}{4}, x_{0}=1$ and $M=2$.

\section{Proofs}

To simplify notation slightly, we sometimes omit showing the explicit dependency of some quantities on $q, d$ and/or $\delta$, e.g., debt, revenues, profits, etc.

\section{D.1 Liquidation Policies}

We use the following notation for the two possible values of the critical quantity $q_{\mathbb{D}}$, see (2),

$$
q_{\mathbb{D}}^{1} \stackrel{\text { def }}{=} \frac{R x_{0}}{R c-\frac{2 s}{M-1+2 s}}, \quad q_{\mathbb{D}}^{2}=\frac{R x_{0}}{R c-\frac{s M}{M+s-1}} .
$$

Proof of Lemma 1. Consider a fixed order quantity $q \leq \frac{x_{0}}{c}$. We seek the firm's liquidation policy as a function of the first period demand realization, $D_{1}=d$. If $d \geq q$, the firm is out of stock, and is indifferent between continuation and liquidation. As such, we consider $d<q$.

The revenues from a liquidation action are given by $x_{\mathcal{R}, \mathscr{L}}(q, d)=X_{\mathcal{R}, \mathscr{L}}(q, d)=(1-s) d+s q$. In view of Assumption 1, the expected revenues from continuation are given by

$$
x_{\mathcal{R}, \mathscr{C}}(q, d)=\mathbb{E}\left[\min \left(d+D_{2}, q\right) \mid D_{1}=d\right]= \begin{cases}\frac{M+1}{2} d, & \text { if } d \leq \frac{q}{M} \\ \frac{q+d}{2}, & \text { if } \frac{q}{M}<d \leq q .\end{cases}
$$

Note that $x_{\mathcal{R}, \mathscr{L}}(q, \cdot)$ is an affine function of $d$ for $d \in[0, q]$, with slope $1-s$. Also, $x_{\mathcal{R}, \mathscr{C}}(q, \cdot)$ is continuous and piece-wise affine in $d$, with slope $\frac{M+1}{2}$ for $d \in\left[0, \frac{q}{M}\right]$, and slope $\frac{1}{2}$ for $d \in\left(\frac{q}{M}, q\right]$.

Furthermore, $x_{\mathcal{R}, \mathscr{L}}(q, 0)=s q>x_{\mathcal{R}, \mathscr{C}}(q, 0)=0$, and $x_{\mathcal{R}, \mathscr{L}}(q, q)=x_{\mathcal{R}, \mathscr{C}}(q, q)$. As such, since $s<\frac{1}{2}$ (by Assumption 2), we immediately have that: $x_{\mathcal{R}, \mathscr{C}}(q, d)>x_{\mathcal{R}, \mathscr{L}}(q, d), \forall d \in$ $\left(\frac{q}{M}, q\right) ; x_{\mathcal{R}, \mathscr{L}}(q, d)>x_{\mathcal{R}, \mathscr{C}}(q, d), \forall d \in(0, \xi) ;$ and $x_{\mathcal{R}, \mathscr{L}}(q, d)<x_{\mathcal{R}, \mathscr{C}}(q, d), \forall d \in\left(\xi, \frac{q}{M}\right)$, where $\xi=\frac{s q}{\frac{M-1}{2}+s}$ is the solution to the equation $\frac{M+1}{2} d=(1-s) d+s q$. It can be readily checked that this exactly corresponds to the liquidation policy $\ell^{\mathrm{fb}}(q, d)$ described in the lemma.

Proof of Lemma 2. Consider a fixed order quantity $q>\frac{x_{0}}{c}$, resulting in a debt $R w(q)=c q-x_{0}$. We seek to characterize the firm's liquidation policy as a function of the realized demand $D_{1}=d$, at the given order quantity $q$.

We first argue that the policy does not depend on whether the covenant is breached, i.e., it is identical in the upper and lower nodes of subgame $\mathbf{S}$ at $t=1$ (see Figure $4(\mathrm{~b})$ ). In the lower node 
of the subgame, when the covenant is not breached, the firm is the sole player responsible for the liquidation/continuation decision, and obtains its optimal policy by solving the problem:

$$
\max _{\ell \in\{0,1\}} \ell \cdot X_{\mathcal{R}, \mathscr{L}}(q, d)+(1-\ell) \cdot \mathbb{E}\left[X_{\mathcal{R}, \mathscr{C}}\left(q, d, D_{2}\right) \mid D_{1}=d\right]
$$

We claim that this problem also yields an optimal policy in the upper node of the subgame, i.e., when there is a covenant breach. Here, the firm solves the following problem:

$$
\max _{\ell \in\{0,1\}}\left[\ell+(1-\ell) \ell_{\mathcal{B}}^{\star}\right] \cdot X_{\mathcal{R}, \mathscr{L}}(q, d)+(1-\ell)\left(1-\ell_{\mathcal{B}}^{\star}\right) \cdot \mathbb{E}\left[X_{\mathcal{R}, \mathscr{C}}\left(q, d, D_{2}\right) \mid D_{1}=d\right]
$$

where $\ell_{\mathcal{B}}^{\star}$ denotes the bank's optimal response. Note that, if $\ell_{\mathcal{B}}^{\star}=1$, the firm's profits are independent of its decision. When $\ell_{\mathcal{B}}^{\star}=0$, the two problems above are equivalent.

The firm's revenues from liquidation as a function of $D_{1}=d$ are

$$
x_{\mathcal{R}, \mathscr{L}}(d)=X_{\mathcal{R}, \mathscr{L}}(q, d)= \begin{cases}0, & \text { if } d \leq d_{\mathrm{S}} \\ (1-s) d+s q-R w, & \text { if } d_{\mathrm{S}}<d \leq q \\ q-R w, & \text { if } q<d .\end{cases}
$$

Here, $d_{\mathrm{S}} \stackrel{\text { def }}{=} \frac{R w-s q}{1-s}$ denotes the demand level for "accounting insolvency," i.e., the demand such that, by liquidating all remaining inventory, the firm is exactly able to replay the entire debt $R w$. As $d$ raises just above $d_{\mathrm{S}}$, the firm's revenues increase linearly, with a slope of $1-s$, and then saturate upon stock-out, at $d=q$. Similarly, its expected revenues from continuation are

$$
x_{\mathcal{R}, \mathscr{C}}(d)=\mathbb{E}\left[X_{\mathcal{R}, \mathscr{C}}\left(q, d, D_{2}\right) \mid D_{1}=d\right]=\mathbb{E}\left[\left(\min \left(d+D_{2}, q\right)-R w\right)^{+} \mid D_{1}=d\right] .
$$

In view of Assumption 1, this expression simplifies to the following cases:

$$
\begin{gathered}
\text { if } R w<\frac{q}{M}, \\
\text { if } R w \geq \frac{q}{M}, \\
x_{\mathcal{R}, \mathscr{C}}(d)=\left\{\begin{array}{ll}
0, & \text { if } d \leq \frac{R w}{M} \\
\frac{M d-R w}{2}, & \text { if } \frac{R w}{M}<d \leq R w \\
\frac{M+1}{2} d-R w, & \text { if } R w<d \leq \frac{q}{M} \\
\frac{d+q}{2}-R w, & \text { if } \frac{q}{M}<d \leq q \\
q-R w, & \text { if } q<d,
\end{array} \quad x_{\mathcal{R}, \mathscr{C}}(d)= \begin{cases}0, & \text { if } d \leq \frac{R w}{M} \\
\frac{M d-R w}{2}, & \text { if } \frac{R w}{M}<d \leq \frac{q}{M} \\
\frac{q-R w}{2}, & \text { if } \frac{q}{M}<d \leq R w \\
\frac{d+q}{2}-R w, & \text { if } R w<d \leq q \\
q-R w, & \text { if } q<d .\end{cases} \right.
\end{gathered}
$$

By continuing, the firm's revenues are exactly zero if $d$ falls below $\frac{R w}{M}$, and then increase in a piece-wise linear fashion, initially with a slope of $\frac{M}{2}$, before saturating upon stock-out, at $d=q$.

The firm's liquidation decision exactly entails comparing $x_{\mathcal{R}, \mathscr{L}}(d)$ with $x_{\mathcal{R}, \mathscr{C}}(d)$, and liquidating 
(continuing) if the former (latter) is strictly larger. Several cases emerge, depending on whether $d_{\mathrm{S}} \gtrless \frac{R w}{M}$ and $1-s \gtrless \frac{M}{2}$. These are treated in Propositions 2 and 3 , and are summarized below. From Proposition 2 , if $M \geq \tilde{M}$, an optimal liquidation policy for the firm is:

$$
\ell_{\mathcal{R}}(q, d)= \begin{cases}\mathbb{1}\left\{d<d_{\mathcal{R}}(q)\right\}, & \text { if } s<s_{\mathbb{D}}^{1} \text { and } q>q_{\mathbb{D}}^{1} \\ \mathbb{1}\left\{d<d^{\mathrm{fb}}(q)\right\}, & \text { otherwise. }\end{cases}
$$

Furthermore, $\ell_{\mathcal{R}}(q, d) \leq \ell(q, d)$ always holds. From Proposition 3 , if $M<\tilde{M}$, an optimal liquidation policy is:

$$
\ell_{\mathcal{R}}(q, d)= \begin{cases}\mathbb{1}\left\{d \in\left[0, \frac{R w(q)}{M}\right) \cup\left(d_{\mathcal{R}}(q), d^{\mathrm{fb}}(q)\right)\right\}, & \text { if } s<s_{\mathbb{D}}^{2} \text { and } q>q_{\mathbb{D}}^{2} \\ \mathbb{1}\left\{d<d^{\mathrm{fb}}(q)\right\}, & \text { otherwise. }\end{cases}
$$

Furthermore, $\ell_{\mathcal{R}}(q, d) \leq \ell^{\mathrm{fb}}(q, d)$ holds, unless $s<\min \left(s_{\mathbb{D}}^{1}, s_{\mathbb{D}}^{2}\right)$ and $q>\max \left(q_{\mathbb{D}}^{1}, q_{\mathbb{D}}^{2}\right)$.

By defining $q_{\mathbb{D}}$ as in the statement of the lemma, it can be readily verified that these policies exactly reduce to the desired ones.

Proof of Lemma 3. We first show that for $M \geq \tilde{M}, q>q_{\mathbb{D}}$ holds if and only if $d^{\mathrm{fb}}<R w$. To see this, note that

$$
R w-d^{\mathrm{fb}}=\frac{\left(s_{\mathbb{D}}^{1}-s\right) q}{2(1-R c)(M-1+2 s)}-R x_{0}=\frac{\left(s_{\mathbb{D}}^{1}-s\right)\left(q-q_{\mathbb{D}}^{1}\right)}{2(1-R c)(M-1+2 s)}
$$

If $q>q_{\mathbb{D}}$ holds, that implies that $q_{\mathbb{D}}=q_{\mathbb{D}}^{1}<q$, as well as $s<s_{\mathbb{D}}^{1}$. Combining these with (13), we get that $d^{\mathrm{fb}}<R w$. On the other hand, if $s \geq s_{\mathbb{D}}^{1}$, then $q \leq q_{\mathbb{D}}=\infty$ holds, and by (13) we also have $d^{\mathrm{fb}}>R w$. If $s<s_{\mathbb{D}}^{1}$ and $q \leq q_{\mathbb{D}}=q_{\mathbb{D}}^{1}$ holds, we also have $d^{\mathrm{fb}} \geq R w$. Hence, $d^{\mathrm{fb}}<R w$ implies $q>q_{\mathbb{D}}$.

We now prove the claim of the Lemma in case $\ell_{\mathcal{R}}$ is followed. The proof in case $\ell^{\mathrm{fb}}$ is followed is similar and is omitted. Consider the case when $q>q_{\mathbb{D}}$. Then, for $M \geq \tilde{M}$, we argued that $d^{\mathrm{fb}}<R w$. However, $\ell_{\mathcal{R}}\left(q, d^{\mathrm{fb}}\right)=0$, i.e., if the first period demand is equal to $d^{\mathrm{fb}}$, there is continuation which would then lead to bankruptcy if the second period demand is zero. For $M<\tilde{M}$, we have that $\ell_{\mathcal{R}}\left(q, \frac{R w}{M}\right)=0$, which again would lead to the same result. As such, $q>q_{\mathbb{D}}$ is a sufficient condition for bankruptcy risk.

To show that $q>q_{\mathbb{D}}$ is also necessary for bankruptcy risk when $M \geq 3$, let us suppose, for the sake of contradiction, that $q \leq q_{\mathbb{D}}$ and that there is bankruptcy risk. First note that

$$
(1-s) d_{\mathrm{S}}=R w-s q<R w-d^{\mathrm{fb}} \leq 0,
$$

where the first inequality holds since $M \geq 3$, and the second since $M \geq \tilde{M}$ and $q \leq q_{\mathbb{D}}$. Since $d_{\mathrm{S}}$ 
is negative, there is no bankruptcy risk at $t=1$. Also, continuation occurs only if $d \geq d^{\mathrm{fb}}$ at $t=1$ and cannot thus lead to bankruptcy.

Finally, to show that $q>q_{\mathbb{D}}$ is not necessary for bankruptcy risk when $M<3$, consider the case when $q=27, c=0.5, s=\frac{7}{16}, R=\frac{13}{8}, M=\frac{5}{4}$ and $x_{0}=1$. Then, we get $s_{\mathbb{D}}^{1}=\frac{13}{24}>s$, $q_{\mathbb{D}}=q_{\mathbb{D}}^{1}=46.8>q, d_{\mathrm{S}}=\frac{136}{9}>0$ and $d^{\mathrm{fb}}=21>d_{\mathrm{S}}$. As such, for this example $q \leq q_{\mathbb{D}}$, but there is bankruptcy risk at $t=1$, since $0<d_{\mathrm{S}}<d^{\mathrm{fb}}$.

Proof of Lemma 4. Consider a fixed order quantity $q>\frac{x_{0}}{c}$, resulting in a debt $R w(q)$, and seek to characterize the banks's liquidation policy as a function of the realized demand $D_{1}=d$, at the given order quantity $q$. In view of Assumption 1, the bank's expected revenues from liquidation $\left(x_{\mathcal{B}, \mathscr{L}}\right)$ and continuation $\left(x_{\mathcal{B}, \mathscr{C}}\right)$ as a function of $D_{1}=d$ are respectively given by:

$$
\begin{aligned}
& x_{\mathcal{B}, \mathscr{L}}(d)=X_{\mathcal{B}, \mathscr{L}}(q, d)= \begin{cases}(1-s) d+s q, & \text { if } d \leq d_{\mathrm{S}} \\
R w, & \text { if } d_{\mathrm{S}}<d,\end{cases} \\
& x_{\mathcal{B}, \mathscr{C}}(d)=\mathbb{E}\left[X_{\mathcal{B}, \mathscr{C}}\left(q, D_{1}, D_{2} \mid D_{1}=d\right)\right]= \begin{cases}\frac{(M+1) d}{2}, & \text { if } d \leq \frac{R w}{M} \\
\frac{d+R w}{2}, & \text { if } \frac{R w}{M}<d \leq R w \\
R w, & \text { if } R w<d,\end{cases}
\end{aligned}
$$

where $d_{\mathrm{S}} \stackrel{\text { def }}{=} \frac{R w-s q}{1-s}$. Note that the bank is indifferent if $d \geq R w$, since, in that case, the first period sales alone are sufficient to cover the entire debt. As such, we focus the discussion of the liquidation decision to cases where $d<R w$.

It can be readily checked that $x_{\mathcal{B}, \mathscr{L}}$ and $x_{\mathcal{B}, \mathscr{C}}$ are both continuous and concave in $d$, with $x_{\mathcal{B}, \mathscr{L}}(0)>x_{\mathcal{B}, \mathscr{C}}(0)$ and $x_{\mathcal{B}, \mathscr{L}}(d)>x_{\mathcal{B}, \mathscr{C}}(d), \forall d \in\left(d_{\mathrm{S}}, R w\right)$. As such, the bank strictly prefers liquidation for "sufficiently low" or "sufficiently high" demands (i.e., larger than $d_{\mathrm{S}}$ ). In fact, by comparing (14a) and (14b), it can be seen that two cases can arise:

Case 1: If $x_{\mathcal{B}, \mathscr{L}}\left(\frac{R w}{M}\right) \geq x_{\mathcal{B}, \mathscr{C}}\left(\frac{R w}{M}\right)$, then $x_{\mathcal{B}, \mathscr{L}}(d) \geq x_{\mathcal{B}, \mathscr{C}}(d), \forall d \in[0, R w)$, so that the optimal liquidation policy for the bank is $\ell_{\mathcal{B}}(q, d)=\mathbb{1}\left\{d<d_{\mathcal{B}}(q)\right\}$.

Case 2: If $x_{\mathcal{B}, \mathscr{L}}\left(\frac{R w}{M}\right)<x_{\mathcal{B}, \mathscr{C}}\left(\frac{R w}{M}\right)$, then there exist two demand levels $\xi_{1} \in\left(0, \frac{R w}{M}\right]$ and $\xi_{2} \in$ $\left[\frac{R w}{M}, d_{\mathrm{S}}\right)$, such that $\ell_{\mathcal{B}}(q, d)=\mathbb{1}\left\{d \in\left[0, \xi_{1}\right) \cup\left(\xi_{2}, R w\right)\right\}$. Here, $\xi_{1}=\frac{s q}{\frac{M-1}{2}+s}=d^{\mathrm{fb}}(q)$ is the solution of the equation $(1-s) d+s q=\frac{(M+1) d}{2}$, and $\xi_{2}=\frac{R w-2 q s}{1-2 s}$ is the solution to the equation $(1-s) d+s q=\frac{d+R w}{2}$.

Finally, note that $x_{\mathcal{B}, \mathscr{L}}\left(\frac{R w}{M}\right)<x_{\mathcal{B}, \mathscr{C}}\left(\frac{R w}{M}\right)$ holds if and only if $\left(\frac{M-1}{2}+s\right) R x_{0}<\left[\left(\frac{M-1}{2}+s\right) R c-\right.$ $M s] q$. Since the left term is always strictly positive, the inequality either never holds (when the term multiplying $q$ is non-positive, which is equivalent to $s \geq s_{\mathcal{B}}$ ), or results in a valid lower bound on $q$, equal to $q_{\mathcal{B}}$ (and valid only for $s<s_{\mathcal{B}}$ ). As such, Case 2 arises if and only if $s<s_{\mathcal{B}}$ and $q>q_{\mathcal{B}}$, which completes the proof. 
Proof of Lemma 5. The proof follows readily by unifying the cases from Lemma 2 and Lemma 4. We omit it for space considerations.

\section{D.1.1 Auxiliary Results}

Proposition 2. For $M \geq \tilde{M} \stackrel{\text { def }}{=} 2(1-s)$, an optimal liquidation policy for the firm is given by (11). Furthermore, $\ell_{\mathcal{R}}(q, d) \leq \ell^{\mathrm{fb}}(q, d)$, for all $q$ and $d$.

Proof. We follow the same notation as in the proof of Lemma 2. Several cases emerge.

Case 1: $\frac{R w}{M}<d_{\mathrm{S}}$ or $\left\{\frac{R w}{M} \leq d_{\mathrm{S}}\right.$ and $\left.M<\tilde{M}\right\}$. By comparing (8) with (9) and (10), it can be readily checked that $x_{\mathcal{R}, \mathscr{C}}(d)>x_{\mathcal{R}, \mathscr{L}}(d), \forall d \in\left(\frac{R w}{M}, \frac{q}{M}\right]$, since $x_{\mathcal{R}, \mathscr{C}}$ has slopes $\frac{M}{2}$ (or $\frac{M+1}{2}$ ), which are larger than $1-s$ (strictly larger when $M<\tilde{M}$, and at least as large, but with $x_{\mathcal{R}, \mathscr{C}}\left(d_{\mathrm{S}}\right)>x_{\mathcal{R}, \mathscr{L}}\left(d_{\mathrm{S}}\right)$ when $M=\tilde{M})$. Combining with Proposition 4, we can see that the optimal liquidation policy for the firm becomes $\ell_{\mathcal{R}}(d)=\mathbb{1}\left\{d<\frac{R w}{M}\right\}$. To check that this corresponds to (11), note from the definition of $d_{\mathcal{R}}$ and $(15 \mathrm{~d})$ that in Case 1 we have $d_{\mathcal{R}}(q)=\frac{R w}{M}$. Furthermore, (15b) and (15c) imply that $s<s_{\mathbb{D}}^{1}$ and $q>q_{\mathbb{D}}^{1}$ holds, which concludes the proof of the case.

Case 2: $M=2(1-s)$ and $\frac{R w}{M}=d_{\mathrm{S}}$. This is a degenerate case, where $x_{\mathcal{R}, \mathscr{C}}(d)=x_{\mathcal{R}, \mathscr{L}}(d), \forall d \in$ $\left[\frac{R w}{M}, \min \left(R w, \frac{q}{M}\right)\right]$, and $x_{\mathcal{R}, \mathscr{C}}(d)>x_{\mathcal{R}, \mathscr{L}}(d), \forall d \in\left(\min \left(R w, \frac{q}{M}\right), q\right)$. As such, any policy of the form $\ell_{\mathcal{R}}(d)=\mathbb{1}\{d<\xi\}$, for some $\xi \in\left[\frac{R w}{M}, \min \left(R w, \frac{q}{M}\right)\right]$ is optimal. Without loss, we can take $\xi=\frac{R w}{M}$, but also $\xi=R w$. Note that, in this case, $d_{\mathcal{R}}(q)=\frac{R w(q)}{M}$, and (15h) implies $d^{\mathrm{fb}}(q)=R w(q)$. Therefore, both branches in (11) yield optimal policies.

Case 3: $\frac{R w}{M}>d_{\mathrm{S}}$. It can be readily seen that $x_{\mathcal{R}, \mathscr{L}}(d)>x_{\mathcal{R}, \mathscr{C}}(d), \forall d \in\left(d_{\mathrm{S}}, \frac{R w}{M}\right]$. Furthermore, by Proposition $4, x_{\mathcal{R}, \mathscr{L}}(d)<x_{\mathcal{R}, \mathscr{C}}(d), \forall d \in\left(\frac{q}{M}, q\right)$. Therefore, $x_{\mathcal{R}, \mathscr{L}}$ and $x_{\mathcal{R}, \mathscr{C}}$ must intersect for some $d \in\left(\frac{R w}{M}, \frac{q}{M}\right)$. We distinguish two sub-cases.

Case 3-A: $R w \geq \frac{q}{M}$. By $(10), x_{\mathcal{R}, \mathscr{L}}$ and $x_{\mathcal{R}, \mathscr{C}}$ are both affine functions on $\left(\frac{R w}{M}, \frac{q}{M}\right)$, with slopes $1-s$ and $\frac{M}{2}$, respectively. We must also have $M<\tilde{M}$ (if $M=\tilde{M}$, the two lines would be parallel and there could be no intersection). In this case, the policy is given by:

$$
x_{\mathcal{R}, \mathscr{L}}(d)>x_{\mathcal{R}, \mathscr{C}}(d), \forall d \in\left[\frac{R w}{M}, \xi\right) \quad \text { and } \quad x_{\mathcal{R}, \mathscr{L}}(d)<x_{\mathcal{R}, \mathscr{C}}(d), \forall d \in\left(\xi, \frac{q}{M}\right)
$$

where $\xi=\frac{2 s q-R w}{M-\tilde{M}}$ is the solution to the equation $\frac{M d-R w}{2}=(1-s) d+s q-R w$.

To see that this exactly corresponds to (11), note first that, by $(15 \mathrm{~d}), \xi=d_{\mathcal{R}}(q)$, which proves that, in Case 3-A,$\ell_{\mathcal{R}}(q, d)=\mathbb{1}\left\{d<d_{\mathcal{R}}(q)\right\}$. Then, (15c) implies that $\left\{s<s_{\mathbb{D}}^{1}\right.$ and $\left.q>q_{\mathbb{D}}^{1}\right\}$ must hold, which concludes the proof.

Case 3-B: $R w<\frac{q}{M}$. In this case, (9) implies that the (unique) demand level $d$ where $x_{\mathcal{R}, \mathscr{L}}(d)=$ $x_{\mathcal{R}, \mathscr{C}}(d)$ can occur for either $d \in\left(\frac{R w}{M}, R w\right]$ or $d \in\left[R w, \frac{q}{M}\right)$. The former (latter) occurs if and only if $x_{\mathcal{R}, \mathscr{C}}(R w)$ is larger (smaller) than $x_{\mathcal{R}, \mathscr{L}}(R w)$.

- If $x_{\mathcal{R}, \mathscr{C}}(R w)>x_{\mathcal{R}, \mathscr{L}}(R w)$, the demand level satisfying $x_{\mathcal{R}, \mathscr{L}}(d)=x_{\mathcal{R}, \mathscr{C}}(d)$ is the solution to 
the equation $\frac{M d-R w}{2}=(1-s) d+s q-R w$, identical to Case 3-A. As such, we again have that $\ell_{\mathcal{R}}(q, d)=\mathbb{1}\left\{d<d_{\mathcal{R}}(q)\right\}$.

- If $x_{\mathcal{R}, \mathscr{C}}(R w) \leq x_{\mathcal{R}, \mathscr{L}}(R w)$, the demand level satisfying $x_{\mathcal{R}, \mathscr{L}}(d)=x_{\mathcal{R}, \mathscr{C}}(d)$ is the solution to the equation $\frac{(M+1) d}{2}=(1-s) d+s q$, which is exactly $\frac{s q}{\frac{M-1}{2}+s}=d^{\mathrm{fb}}$. As such, we conclude that $\ell_{\mathcal{R}}(q, d)=\mathbb{1}\left\{d<d^{\mathrm{fb}}(q)\right\}$.

To see that this corresponds to (11), note that (15b) implies that $x_{\mathcal{R}, \mathscr{C}}(R w)>x_{\mathcal{R}, \mathscr{L}}(R w)$ holds if and only if $\left\{s<s_{\mathbb{D}}^{1}\right.$ and $\left.q>q_{\mathbb{D}}^{1}\right\}$, which is exactly what is required for (11).

Finally, to see that $\ell_{\mathcal{R}}(q, d) \leq \ell^{\mathrm{fb}}(q, d)$ holds for all $q$ and $d$, note that (15f) and (15g) imply that $d^{\mathrm{fb}}(q)<d_{\mathcal{R}}(q)$ can only hold if $s<s_{\mathbb{D}}^{1}$ and $q>q_{\mathbb{D}}^{1}$. As such, (11) directly leads to the desired conclusion.

Proposition 3. For $M<\tilde{M} \stackrel{\text { def }}{=} 2(1-s)$, an optimal liquidation policy for the firm is given by (12). Furthermore, the following modified policy $\widehat{\ell_{\mathcal{R}}}(q, d)$ is also optimal for the firm, and satisfies $\widehat{\ell_{\mathcal{R}}}(q, d) \leq \ell^{\mathrm{fb}}(q, d)$ for any $q$ and any $d$ :

$$
\widehat{\ell_{\mathcal{R}}}(q, d)= \begin{cases}0 & \text { if }\{M<\tilde{M}\} \text { and }\left\{s<\min \left(s_{\mathbb{D}}^{1}, s_{\mathbb{D}}^{2}\right)\right\} \text { and }\left\{q>\max \left(q_{\mathbb{D}}^{1}, q_{\mathbb{D}}^{2}\right)\right\} \\ \ell_{\mathcal{R}}(q, d) & \text { otherwise. }\end{cases}
$$

Proof. We follow the same notation as in the proof of Lemma 2. Several cases emerge.

Case 1: $\frac{R w}{M} \leq d_{\mathrm{S}}$. In this case, we have that $x_{\mathcal{R}, \mathscr{C}}\left(d_{\mathrm{S}}\right) \geq x_{\mathcal{R}, \mathscr{L}}\left(d_{\mathrm{S}}\right)=0$, and (15a) also implies that $\left\{s<s_{\mathbb{D}}^{2}\right\}$ and $q \geq q_{\mathbb{D}}^{2}$, i.e., we are in the first case of (12). Two sub-cases emerge.

Case 1-A: $R w \geq \frac{q}{M}$. In this case, (10) and Proposition 4 imply that $x_{\mathcal{R}, \mathscr{C}}(d)>x_{\mathcal{R}, \mathscr{L}}(d), \forall d \in$ $\left(\frac{R w}{M}, q\right)$. This is equivalent to the optimal liquidation policy $\ell_{\mathcal{R}}(q, d)=\mathbb{1}\left\{d<\frac{R w(q)}{M}\right\}$. To see that this is equivalent to (12), note that Case 1-A and (15c) imply that $s<s_{\mathbb{D}}^{1}$ and $q>q_{\mathbb{D}}^{1}$, which, by (15f), implies that $d^{\mathrm{fb}}(q) \leq d_{\mathcal{R}}(q)$, so that $\left(d_{\mathcal{R}}(q), d^{\mathrm{fb}}(q)\right)=\emptyset$.

Case 1-B: $R w<\frac{q}{M}$. Here, we have $x_{\mathcal{R}, \mathscr{C}}(d)>x_{\mathcal{R}, \mathscr{L}}(d), \forall d \in\left(\frac{R w}{M}, d_{\mathrm{S}}\right] \cup\left[\frac{q}{M}, q\right)$. In the interval $\left(d_{\mathrm{S}}, \frac{q}{M}\right), x_{\mathcal{R}, \mathscr{C}}$ is piecewise-affine, with two pieces, and $x_{\mathcal{R}, \mathscr{L}}$ is affine. Two possibilities emerge:

- If $x_{\mathcal{R}, \mathscr{C}}(R w) \geq x_{\mathcal{R}, \mathscr{L}}(R w)$, then $x_{\mathcal{R}, \mathscr{C}}(d) \geq x_{\mathcal{R}, \mathscr{L}}(d), \forall d \in\left(\frac{R w}{M}, q\right)$, and $x_{\mathcal{R}, \mathscr{C}}$ and $x_{\mathcal{R}, \mathscr{L}}$ have at most one point of tangency in $\left(d_{\mathrm{S}}, \frac{q}{M}\right)$, so the liquidation policy becomes $\ell_{\mathcal{R}}(q, d)=\mathbb{1}\{d<$ $\left.\frac{R w(q)}{M}\right\}$. To see that this corresponds to (12), note that (15b) implies that $\left\{s<s_{\mathbb{D}}^{1}\right.$ and $\left.q \geq q_{\mathbb{D}}^{1}\right\}$, which, by (15f), implies that $\left(d_{\mathcal{R}}(q), d^{\mathrm{fb}}(q)\right)=\emptyset$.

- If $x_{\mathcal{R}, \mathscr{C}}(R w)<x_{\mathcal{R}, \mathscr{L}}(R w)$, then $x_{\mathcal{R}, \mathscr{C}}$ and $x_{\mathcal{R}, \mathscr{L}}$ have two intersection points. One such point is given by the solution to the equation $\frac{M d-R w}{2}=(1-s) d+s q-R w$, i.e., $\frac{2 s q-R w}{M-2(1-s)}$. By $(15 \mathrm{e})$, this is exactly $d_{\mathcal{R}}(q)$. The other such point is the solution to the equation $\frac{(M+1) d}{2}=$ $(1-s) d+s q$, which is exactly $\frac{s q}{\frac{M-1}{2}+s}=d^{\mathrm{fb}}$. By $(15 \mathrm{~b})$ and $(15 \mathrm{f})$, we also have $d^{\mathrm{fb}}(q)>d_{\mathcal{R}}(q)$, 
so that the liquidation policy is $\ell_{\mathcal{R}}(q, d)=\mathbb{1}\left\{d \in\left[0, \frac{R w(q)}{M}\right) \cup\left(d_{\mathcal{R}}(q), d^{\mathrm{fb}}(q)\right)\right\}$, which exactly corresponds to (12).

Case 2: $\frac{R w}{M}>d_{\mathrm{S}}$. In this case, we claim that $R w<\frac{q}{M}$. To see this, note that $R w \geq \frac{q}{M}$ and $x_{\mathcal{R}, \mathscr{L}}\left(\frac{w R}{M}\right)>x_{\mathcal{R}, \mathscr{C}}\left(\frac{w R}{M}\right)=0$ would imply, through $(10)$, that $x_{\mathcal{R}, \mathscr{L}}\left(\frac{q}{M}\right)>x_{\mathcal{R}, \mathscr{C}}\left(\frac{q}{M}\right)$, since the slope of the liquidation profits $(1-s)$ is greater than the slope of the continuation profits $\left(\frac{M}{2}\right)$. The latter would be in direct contradiction with Proposition 4. Since $R w<\frac{q}{M}$, a similar reasoning to the one above applied to the profits in $(9)$ shows that $x_{\mathcal{R}, \mathscr{L}}(R w)>x_{\mathcal{R}, \mathscr{C}}(R w)$, so that

$$
x_{\mathcal{R}, \mathscr{L}}(d)>x_{\mathcal{R}, \mathscr{C}}(d), \forall d \in(0, \xi) \quad \text { and } \quad x_{\mathcal{R}, \mathscr{L}}(d)<x_{\mathcal{R}, \mathscr{C}}(d), \forall d \in\left(\xi, \frac{q}{M}\right)
$$

where $\xi$ is the solution to the equation $\frac{(M+1) d}{2}=(1-s) d+s q$, which is exactly $\frac{s q}{\frac{M-1}{2}+s}=d^{\mathrm{fb}}$. As such, $\ell_{\mathcal{R}}(q, d)=\mathbb{1}\left\{d<d^{\mathrm{fb}}(q)\right\}$. To see that this exactly corresponds to (12), note that $\frac{R w}{M}>d_{\mathrm{S}}$ and (15a) imply that $\left\{s<s_{\mathbb{D}}^{2}\right.$ and $\left.q>q_{\mathbb{D}}^{2}\right\}$ cannot hold.

To see that the modified policy $\widehat{\ell_{\mathcal{R}}}(q, d)$ satisfies $\widehat{\ell_{\mathcal{R}}}(q, d) \leq \ell^{\mathrm{fb}}(q, d)$, note from (12) that the only case where $\ell_{\mathcal{R}}(q, d) \leq \ell^{\mathrm{fb}}(q, d)$ might not hold is when $s<s_{\mathbb{D}}^{2}, q>q_{\mathbb{D}}^{2}$, and $d^{\mathrm{fb}}(q)<\frac{R w}{M}$, i.e., by $(15 \mathrm{~g}), s<s_{\mathbb{D}}^{1}, q>q_{\mathbb{D}}^{1}$. In this case, $\ell_{\mathcal{R}}(q, d)=\mathbb{1}\left\{d<\frac{w R}{M}\right\}$. However, by (15a), note that $\frac{w R}{M}<d_{\mathrm{S}}$, so that the firm is actually indifferent between liquidation and continuation, so that the policy $\widehat{\ell_{\mathcal{R}}}(q, d)$ is also optimal.

Proposition 4. $x_{\mathcal{R}, \mathscr{C}}(d)>x_{\mathcal{R}, \mathscr{L}}(d), \forall d \in\left[\frac{q}{M}, q\right)$, i.e., $\ell_{\mathcal{R}}(q, d)=\ell^{\mathrm{fb}}(q, d)=0, \forall d \in\left[\frac{q}{M}, q\right)$.

Proof. The result trivially holds if $\frac{q}{M}<d_{\mathrm{S}}$, so we only consider the reverse case. We distinguish two cases, depending on whether $R w \gtrless \frac{q}{M}$.

If $R w<\frac{q}{M}$, note from (9) that $x_{\mathcal{R}, \mathscr{C}}$ and $x_{\mathcal{R}, \mathscr{L}}$ are affine in $d \in\left[\frac{q}{M}, q\right]$, and $x_{\mathcal{R}, \mathscr{C}}(q)=x_{\mathcal{R}, \mathscr{L}}(q)$. Showing the main result is thus equivalent to arguing $x_{\mathcal{R}, \mathscr{C}}\left(\frac{q}{M}\right)>x_{\mathcal{R}, \mathscr{L}}\left(\frac{q}{M}\right)$, which holds, since:

$$
x_{\mathcal{R}, \mathscr{C}}\left(\frac{q}{M}\right)-x_{\mathcal{R}, \mathscr{L}}\left(\frac{q}{M}\right)=\frac{q(M-1)(1-2 s)}{2 M}>0, \text { since } s<\frac{1}{2} \text { and } M>1
$$

If $R w \geq \frac{q}{M}$, note from (10) that $x_{\mathcal{R}, \mathscr{C}}$ is constant for $d \in\left[\frac{q}{M}, R w\right]$, and affine, with slope $\frac{1}{2}$ for $d \in(R w, q]$. Since $x_{\mathcal{R}, \mathscr{L}}$ is affine for $d \in\left(\frac{q}{M}, q\right]$, with slope $0 \leq s<\frac{1}{2}$, and $x_{\mathcal{R}, \mathscr{C}}(q)=x_{\mathcal{R}, \mathscr{L}}(q)$, to argue the result, it suffices to show that $x_{\mathcal{R}, \mathscr{C}}(R w)>x_{\mathcal{R}, \mathscr{L}}(R w)$, which holds, since

$$
x_{\mathcal{R}, \mathscr{C}}(R w)-x_{\mathcal{R}, \mathscr{L}}(R w)=(q-R w)\left(\frac{1}{2}-s\right)>0, \text { since } q>R w \text { and } s<\frac{1}{2}
$$


Proposition 5. We have: $:^{20}$

$$
\begin{aligned}
\frac{R w}{M} \stackrel{(<)}{\leq} d_{\mathrm{S}} & \Leftrightarrow\left\{s<s_{\mathbb{D}}^{2} \text { and } q \stackrel{(>)}{\geq} q_{\mathbb{D}}^{2}\right\} \\
\text { If } R w<\frac{q}{M}, \text { then } x_{\mathcal{R}, \mathscr{C}}(R w) \stackrel{(>)}{\geq} x_{\mathcal{R}, \mathscr{L}}(R w) & \Leftrightarrow\left\{s<s_{\mathbb{D}}^{1} \text { and } q \stackrel{(>)}{\geq} q_{\mathbb{D}}^{1}\right\} \\
R w \geq \frac{q}{M} & \Rightarrow\left\{s<s_{\mathbb{D}}^{1} \text { and } q>q_{\mathbb{D}}^{1}\right\} \\
\text { If } M>2(1-s) \text {, then } d_{\mathcal{R}}(q)=\frac{R w(q)}{M} & \Leftrightarrow \frac{R w}{M} \leq d_{\mathrm{S}} \\
\text { If } M<2(1-s), \text { then } d_{\mathcal{R}}(q)=\frac{R w(q)}{M} & \Leftrightarrow \frac{R w}{M} \geq d_{\mathrm{S}} \\
\text { If } M<2(1-s), d^{\mathrm{fb}}(q) \stackrel{(<)}{\leq} \frac{2 s q-R w}{M-2(1-s)} & \Leftrightarrow\left\{s<s_{\mathbb{D}}^{1} \text { and } q \geq q_{\mathbb{D}}^{1}\right\} \\
\left\{M=2(1-s) \text { and } \frac{R w}{M}=d_{\mathrm{S}}\right\} & \Rightarrow d^{\mathrm{fb}}(q)=R w(q) .
\end{aligned}
$$

Proof. Throughout this proof, recall that $0<R c \leq 1, M>1$, and $s<\frac{1}{2}$.

To prove (15a), note that $\frac{R w}{M} \leq d_{\mathrm{S}}$ holds if and only if:

$$
\frac{x_{0} R(M+s-1)}{M(1-s)} \leq\left(\frac{c R-s}{1-s}-\frac{c R}{M}\right) q
$$

Since the left term is always strictly positive, the inequality either never holds (when the term multiplying $q$ is non-positive, which is equivalent to $s \geq s_{\mathbb{D}}^{2}$ ), or results in a valid lower bound on $q$ (equal to $q_{\mathbb{D}}^{2}$, and valid only for $s<s_{\mathbb{D}}^{2}$ ). The strict version of the inequality follows similarly.

To prove $(15 \mathrm{~b})$, note that, by $(9), x_{\mathcal{R}, \mathscr{C}}(R w) \geq x_{\mathcal{R}, \mathscr{L}}(R w)$ holds if and only if:

$$
\frac{M-1+2 s}{2} R x_{0} \leq\left(\frac{M-1+2 s}{2} R c-s\right) q .
$$

Since the left term is always strictly positive, the inequality either never holds (when the term multiplying $q$ is non-positive, which is equivalent to $s \geq s_{\mathbb{D}}^{1}$ ), or results in a valid lower bound on $q$ (equal to $q_{\mathbb{D}}^{1}$, and valid only for $s<s_{\mathbb{D}}^{1}$ ). The strict version of the inequality follows similarly.

To prove (15c), note first that $R w \geq \frac{q}{M}$ is equivalent to

$$
(M R c-1) q \geq M R x_{0} \Leftrightarrow\left\{M R c>1 \quad \text { and } \quad q \geq \frac{M R x_{0}}{M R c-1}\right\} .
$$

We first show that $M R c>1$ implies $s<s_{\mathbb{D}}^{1}$. To this end, note that the latter is equivalent to $R c>\frac{2 s}{M+2 s-1}$. But $M R c>1$ implies $R c>\frac{1}{M}$, and $\frac{1}{M}>\frac{2 s}{M+2 s-1} \quad \Leftrightarrow \quad M-1>2 s(M-1) \quad \Leftrightarrow$

\footnotetext{
${ }^{20}$ In order to save space, since we require both the strict and non-strict versions of the inequalities, we use a compact notation that shows in parenthesis the alternate versions.
} 
$s<\frac{1}{2}$, which is always true. Therefore, $s<s_{\mathbb{D}}^{1}$, which implies, by $(16)$, that $q_{\mathbb{D}}^{1}>0$. To see that

$q>q_{\mathbb{D}}^{1}$, note that (17) also requires $q>\frac{M R x_{0}}{M R c-1}$, and we always have: $\frac{M R x_{0}}{M R c-1}>\frac{R x_{0}}{R c-\frac{2 s}{M-1+2 s}} \Leftrightarrow$ $M-1+2 s>2 M s \quad \Leftrightarrow \quad 1>2 s$.

To prove $(15 \mathrm{~d})$ and $(15 \mathrm{e})$, note that $d_{\mathcal{R}}=\frac{R w}{M}$ holds if and only if $\frac{R w}{M} \geq \frac{2 s q-R w}{M-2+2 s}$. The latter inequality is exactly equivalent to

$$
\begin{array}{ll}
(M+s-1) R x_{0} \leq[M(R c-s)-R c(1-s)] q & \text { if } M>2(1-s) \\
(M+s-1) R x_{0} \geq[M(R c-s)-R c(1-s)] q & \text { if } M<2(1-s) .
\end{array}
$$

The former condition is exactly equivalent to condition (17), i.e., to $\frac{R w}{M} \leq d_{\mathrm{S}}$. Similarly, the latter is equivalent to $\frac{R w}{M} \geq d_{\mathrm{S}}$.

To prove (15f), note that the relation is equivalent, under $M<2(s-1)$, to $R x_{0}(M+2 s-1) \leq$ $[(M-1) R c-2 s(1-R c)] q$, which is exactly (16). By the same arguments as in (15b), this is equivalent to $\left\{s<s_{\mathbb{D}}^{1}\right.$ and $\left.q \geq q_{\mathbb{D}}^{1}\right\}$.

To prove $(15 \mathrm{~g})$, note that the condition is equivalent to $R x_{0}(M+2 s-1) \leq[(M+2 s-1) R c-$ $2 s M] q$. Comparing this with (16), it can be seen that the left-hand-sides are identical, while the term multiplying $q$ here is smaller than in (16). As such, if the relation above holds, it must be that $s<s_{\mathbb{D}}^{1}$ and $q>q_{\mathbb{D}}^{1}$.

To prove $(15 \mathrm{~h})$, note that $d^{\mathrm{fb}}(q)-R w$ becomes equal in this case to:

$$
\frac{2 s q}{M+2 s-1}-R w=s q\left[\frac{2}{M+2 s-1}-\frac{M}{M+s-1}\right]=0
$$

where the first equality follows by expressing $R w$ from the identity $R w=\frac{R w-s q}{1-s}$, and the second follows by using $M=2(1-s)$.

\section{D.2 Perfect Competition}

To simplify the exposition, we introduce some notation. Let $R: \mathbb{R} \rightarrow[1, \infty)$ and $\delta: \mathbb{R} \rightarrow \mathbb{R}_{+}$ be functions that map an order quantity to an interest rate and a covenant demand threshold requirement. In this section, we denote a contract under these terms with $\kappa=(R, \delta)$, and the set of all such competitively priced loans with $\mathbb{K}$.

Consider also the following three particular competitively priced contracts of interest:

(a) $\kappa_{0}=\left(R_{0}, \delta_{0}\right)$, with $\delta_{0}(q) \equiv 0$;

(b) $\kappa_{1}=\left(R_{1}, \delta_{1}\right)$, with $\delta_{1}(q) \equiv \frac{2 s q}{M+2 s-1}$;

(c) $\left(R^{\mathrm{fb}}, 0\right)$, offered to a firm following the first best actions, i.e., ordering $q^{\mathrm{fb}}$, and liquidating according to $\ell^{\mathrm{fb}}$. Let $q_{\mathbb{D}}^{\mathrm{fb}}$ be as in Lemma 2, calculated using $R^{\mathrm{fb}}$.

Finally, we derive the first order optimality conditions (FOC) that are necessary and sufficient 
for the first best order quantity $q^{\mathrm{fb}}$. Note that, using Lemma 1, we can express (1) as

$$
\begin{aligned}
V(q)=\int_{0}^{d^{\mathrm{fb}}}(s q+ & (1-s) u) f_{1}(u) d u+\frac{1}{2} \int_{d^{\mathrm{fb}}}^{\frac{q}{M}}(M+1) u f_{1}(u) d u \\
& +\frac{1}{2} \int_{\frac{q}{M}}^{q}(q+u) f_{1}(u) d u+\int_{q}^{\infty} q f_{1}(u) d u-c q, \quad \forall q \geq 0 .
\end{aligned}
$$

Thus, the FOC is: $1+s F_{1}\left(d^{\mathrm{fb}}\right)=\frac{1}{2} F_{1}(q / M)+\frac{1}{2} F_{1}(q)+c$.

We present the proofs in the same order in which theorems appear in the next. However, the proofs of Theorems 1 and 2 rely on the proof of Theorem 4.

Proof of Theorem 1. (i) If $x_{0} \geq \tilde{x}_{0}$, by Theorem 4(b) we have that $q^{\mathrm{fb}} \leq q_{\mathbb{D}}^{\mathrm{fb}}$. By Proposition 6 , the firm prefers $\kappa_{0}$, which does not have covenants, orders $q^{\mathrm{fb}}$ and follows $\ell^{\mathrm{fb}}$.

(ii) Suppose now that $x_{0}<\tilde{x}_{0}$. Let $g\left(q, x_{0}\right)$ denote the threshold $q_{\mathbb{D}}$ evaluated under the interest rate $R_{0}(q)$ for a firm with initial capital of $x_{0}$, i.e.,

$$
g\left(q, x_{0}\right) \stackrel{\text { def }}{=} \frac{R_{0}(q) x_{0}}{R_{0}(q) c-\theta}
$$

where $\theta$ is defined as in the proof of Theorem 4. Arguing in precisely the same way as in (19), since $R_{0}(q)$ and $R^{\mathrm{fb}}$ have the same monotonicity in $x_{0}$, by Proposition 7 , we have that $g\left(q^{\mathrm{fb}}, x_{0}\right)$ is increasing in $x_{0}$. Based on Theorem $4(\mathrm{~b})$ and Proposition $6, g\left(q^{\mathrm{fb}}, \tilde{x}_{0}\right)=q^{\mathrm{fb}}$. By the monotonicity of $g\left(q^{\mathrm{fb}}, x_{0}\right)$ with respect to $x_{0}$, we then get that

$$
q^{\mathrm{fb}}=g\left(q^{\mathrm{fb}}, \tilde{x}_{0}\right)>g\left(q^{\mathrm{fb}}, x_{0}\right)
$$

Suppose now that in equilibrium the firm orders $q^{\star}$ and follows $\ell^{\mathrm{fb}}$. Then, by Lemma 2 ,

$$
q^{\star} \leq g\left(q^{\star}, x_{0}\right)
$$

Note also that $g\left(q, x_{0}\right)$ is decreasing in $q$. To see this,

$$
\frac{\partial g\left(q, x_{0}\right)}{\partial q}=\frac{\partial g\left(q, x_{0}\right)}{\partial R_{0}} \frac{\partial R_{0}}{\partial w} \frac{\partial w}{\partial q}<0
$$

since the first multiplier above is negative, the second is positive by Proposition 7 , and the third is equal to $c$. If $q^{\star} \geq q^{\mathrm{fb}}$, we get $q^{\star} \geq q^{\mathrm{fb}}>g\left(q^{\mathrm{fb}}, x_{0}\right)>g\left(q^{\star}, x_{0}\right)$, a contradiction. Hence, $q^{\star}<q^{\mathrm{fb}}$.

Finally, if the firm follows $\ell_{\mathcal{R}}<\ell^{\mathrm{fb}}$ in equilibrium, then, since its expected profits are equal to $V$ when following $\ell_{\mathcal{R}}, q^{\star}$ solves the FOC $s F_{1}\left(d_{\mathcal{R}}(q)\right)+1=\frac{1}{2} F_{1}(q)+\frac{1}{2} F_{1}\left(\frac{q}{M}\right)+c$. Since $q^{\mathrm{fb}}$ solves the FOC for $d^{\mathrm{fb}}>d_{\mathcal{R}}$, and $F_{1}, d^{\mathrm{fb}}$ and $d_{\mathcal{R}}$ are all increasing in $q$, we conclude that $q^{\star}<q^{\mathrm{fb}}$.

Proof of Theorem 2. The proof follows from Proposition 6. 
Proof of Theorem 3. By Proposition 6, when covenants are present, contract $\kappa_{1}$ is optimal and thus the corresponding covenant demand threshold is exactly equal to $\delta^{\star}=\frac{2 s q^{\mathrm{fb}}}{M+2 s-1}$.

To prove the comparative statics, it can be readily checked that:

$$
\begin{aligned}
& \operatorname{sgn}\left(\frac{\partial \delta^{\star}}{\partial s}\right)=\operatorname{sgn}\left[(\underbrace{(M-1) 2 q^{\mathrm{fb}}}_{\geq 0}+\underbrace{2 s(M+2 s-1)}_{\geq 0} \frac{\partial q^{\mathrm{fb}}}{\partial s}]\right. \\
& \operatorname{sgn}\left(\frac{\partial \delta^{\star}}{\partial c}\right)=\operatorname{sgn}\left(\frac{\partial q^{\mathrm{fb}}}{\partial c}\right) .
\end{aligned}
$$

Recall that $q^{\mathrm{fb}}$ is the maximizer of $V$ given by (1). It can be readily checked that:

$$
\frac{\partial^{2} V}{\partial q \partial s}=F_{1}\left(\frac{2 s q}{M+2 s-1}\right)+s f_{1}\left(\frac{2 s q}{M+2 s-1}\right) \frac{\partial d^{\mathrm{fb}}}{\partial s} \geq 0
$$

since $\frac{\partial d^{\mathrm{fb}}}{\partial s}=\frac{2 q(M-1)}{(M+2 s-1)^{2}} \geq 0$. As such, $V$ is supermodular in $(q, s)$ on the lattice $\mathbb{R}^{2}$, so that $q^{\mathrm{fb}}$ is increasing in $s$ (see, e.g., Topkis 1998). Similarly, it can be checked that $\frac{\partial d^{\mathrm{fb}}}{\partial c} \leq 0$, so that $q^{\mathrm{fb}}$ is decreasing in $c$.

Proof of Theorem 4. We start with the following useful intermediate result. We prove that a covenant is necessarily included if and only if there is liquidation conflict. To this end, note that Proposition 6 implies that, in equilibrium, the order quantity is always $q^{\mathrm{fb}}$, and the liquidation policy followed is $\ell^{\mathrm{fb}}$. By definition (see Lemma 5), liquidation conflict exists if and only if $q^{\mathrm{fb}}>q_{\mathbb{D}}^{\mathrm{fb}}$. The proof is complete by invoking again Proposition 6 .

To prove the dependence on $x_{0}$, in view of Proposition 6, it is sufficient to show that if $q^{\mathrm{fb}}>q_{\mathbb{D}}^{\mathrm{fb}}$ holds for some $\bar{x}_{0}$, it also holds for any $x_{0}<\bar{x}_{0}$. Since $q^{\mathrm{fb}}$ does not depend on $x_{0}$, it then suffices to show that $q_{\mathbb{D}}^{\mathrm{fb}}$ is increasing in $x_{0}$. Note that

$$
\operatorname{sgn}\left(\frac{d q_{\mathbb{D}}^{\mathrm{fb}}}{d x_{0}}\right)=\operatorname{sgn}\left[\frac{d}{d x_{0}}\left(\frac{R^{\mathrm{fb}} x_{0}}{R^{\mathrm{fb}} c-\theta}\right)\right]=\operatorname{sgn}\left[R^{\mathrm{fb}}\left(R^{\mathrm{fb}} c-\theta\right)-x_{0} \theta \frac{\partial R^{\mathrm{fb}}}{\partial x_{0}}\right],
$$

where $\theta$ is independent of $x_{0}$ (see (2)). Thus, since $R^{\mathrm{fb}} c-\theta>0$, and $R^{\mathrm{fb}}$ is decreasing in $x_{0}$, by Proposition 7 , the proof is complete.

The connection with bankruptcy follows from the results of Proposition 6 and Lemma 3.

\section{D.2.1 Auxiliary Results}

Proposition 6. In equilibrium, the firm prefers $\kappa_{0}\left(\kappa_{1}\right)$ to any other $\kappa \in \mathbb{K}$ if $q^{\mathrm{fb}} \leq q_{\mathbb{D}}^{\mathrm{fb}}\left(q^{\mathrm{fb}}>q_{\mathbb{D}}^{\mathrm{fb}}\right)$. Moreover, in equilibrium, it orders $q^{\mathrm{fb}}$, and liquidates according to $\ell^{\mathrm{fb}}$.

Proof. Let $\pi_{\mathcal{P}}(q ;(R, \delta))$ be the expected profits of agent $\mathcal{P} \in\{\mathcal{R}, \mathcal{B}\}$ in equilibrium when the order quantity is $q$ and the contract offered has an interest rate $R$ and a covenant of $\delta$. Then, under perfect competition $\pi_{\mathcal{B}}\left(q ;\left(R_{i}(q), \delta_{i}(q)\right)\right)=0$, for all $q \geq 0, i \in\{0,1\}$. 
Let $x(q ; \ell)$ be the net expected revenues at the end of the game, generated by sales and possible liquidation of the inventory, under an order quantity $q$ and a liquidation policy $\ell$. We can express the firm's profits as the net expected revenues minus inventory purchasing costs minus the bank's profits, that is, for any $\kappa=(R, \delta) \in \mathbb{K}$

$$
\pi_{\mathcal{R}}(q ;(R(q), \delta(q)))=x\left(q ; \ell_{\kappa, q}\right)-c q-\pi_{\mathcal{B}}(q ;(R(q), \delta(q)))=x\left(q ; \ell_{\kappa, q}\right)-c q,
$$

where $\ell_{\kappa, q}$ is the liquidation policy followed in equilibrium under quantity $q$ and contract $\kappa$.

Let $\pi_{\mathcal{R}}^{\kappa \star}$ be the firm's optimal expected profits under contract $\kappa \in \mathbb{K}$. Then,

$$
\pi_{\mathcal{R}}^{\kappa \star}=\max _{q} \pi_{\mathcal{R}}(q ;(R(q), \delta(q)))=\max _{q}\left\{x\left(q ; \ell_{\kappa, q}\right)-c q\right\}
$$

Case 1: $q^{\mathrm{fb}} \leq q_{\mathbb{D}}^{\mathrm{fb}}$. We argue that $R_{0}\left(q^{\mathrm{fb}}\right)=R^{\mathrm{fb}}$. To see this, note that if the firm wishes to order $q^{\mathrm{fb}}$ and the bank offers the contract $\left(R^{\mathrm{fb}}, 0\right)$, then, since $q^{\mathrm{fb}} \leq q_{\mathbb{D}}^{\mathrm{fb}}$, the firm will follow $\ell^{\mathrm{fb}}$, according to Lemma 2. Consequently, we also have $\ell_{\kappa_{0}, q^{\mathrm{fb}}}=\ell^{\mathrm{fb}}$, and

$$
\begin{aligned}
\pi_{\mathcal{R}}^{\kappa_{0} \star} & =\max _{q}\left\{x\left(q ; \ell_{\kappa_{0}, q}\right)-c q\right\} \\
& \geq x\left(q^{\mathrm{fb}} ; \ell_{\kappa_{0}, q^{\mathrm{fb}}}\right)-c q^{\mathrm{fb}} \\
& =x\left(q^{\mathrm{fb}} ; \ell^{\mathrm{fb}}\right)-c q^{\mathrm{fb}} \\
& \geq \sup _{\kappa \in \mathbb{K}} \max _{q}\left\{x\left(q ; \ell_{\kappa, q}\right)-c q\right\} \\
& =\sup _{\kappa \in \mathbb{K}} \pi_{\mathcal{R}}^{\kappa \star} \geq \pi_{\mathcal{R}}^{\kappa_{0} \star},
\end{aligned}
$$

where the second inequality follows from the optimality of ordering $q^{\mathrm{fb}}$ and following $\ell^{\mathrm{fb}}$ as first best and the third inequality from the fact that $\kappa_{0} \in \mathbb{K}$.

Case 2: $q^{\mathrm{fb}}>q_{\mathbb{D}}^{\mathrm{fb}}$. Consider contract $\kappa_{1}$. Then, by the choice of $\delta_{1}(q)$, we have that $\delta_{1}(q)=d^{\mathrm{fb}}(q)$. According to Lemma 4 , the bank will prefer liquidation for any $d_{1}<d^{\mathrm{fb}}(q)$. Moreover, since $\ell_{\mathcal{R}} \leq \ell^{\mathrm{fb}}$ (see Lemma 2), we have that $\ell_{\kappa_{1}, q}=\ell^{\mathrm{fb}}$. Hence,

$$
\begin{aligned}
\pi_{\mathcal{R}}^{\kappa_{1} \star} & =\max _{q}\left\{x\left(q ; \ell_{\kappa_{1}, q}\right)-c q\right\} \\
& =\max _{q}\left\{x\left(q ; \ell^{\mathrm{fb}}\right)-c q\right\} \\
& =x\left(q^{\mathrm{fb}} ; \ell^{\mathrm{fb}}\right)-c q^{\mathrm{fb}} \\
& \geq \sup _{\kappa \in \mathbb{K}} \pi_{\mathcal{R}}^{\kappa \star} \geq \pi_{\mathcal{R}}^{\kappa_{1} \star} .
\end{aligned}
$$

Proposition 7. For any fixed $q, R_{0}(q)$ is decreasing in $x_{0}$. Similarly, $R^{\mathrm{fb}}$ is decreasing in $x_{0}$.

Proof. We provide a unifying proof for both quantities. Let $R$ below denote either $R_{0}(q)$ or $R^{\mathrm{fb}}$. 
Let $\zeta$ denote the probability of bankruptcy, and $x_{b r}$ denote the expected channel revenues conditional on bankruptcy. By definition, $x_{b r}<R w$. The bank's expected profit can be written as

$$
\pi_{\mathcal{B}}=R w(1-\zeta)+x_{b r} \zeta-w \equiv 0
$$

As a side remark, this implicit equation yields the $R$ that should be charged by the bank. Using the implicit function theorem, this then yields:

$$
\underbrace{R(1-\zeta)-1}_{\leq 0}-\underbrace{\left(R w-x_{b r}\right)}_{>0} \underbrace{\frac{\partial \zeta}{\partial w}}_{\geq 0}+w(1-\zeta) \frac{\partial R}{\partial w}=0 .
$$

Above, the first term is negative due to the identity $\pi_{\mathcal{B}} \equiv 0$, and $\frac{\partial \zeta}{\partial w} \geq 0$ since, ceteris paribus, the probability of bankruptcy increases in the size of the principal. Since $\frac{\partial R}{\partial x_{0}}=-\frac{\partial R}{\partial w}$, the proof is complete.

\section{D.3 Partial Liquidation and Replenishment}

Let $y_{1} \stackrel{\text { def }}{=}\left(q-d_{1}\right)^{+}$be the leftover inventory after the first-period sales and $y$ be the inventory position at the beginning of period $t=2$, after the liquidation or replenishment actions. Let $x_{\mathcal{P}}$ be the expected cash flow of player $\mathcal{P} \in\{\mathcal{R}, \mathcal{B}\}$ at the end of period $t=2$. We denote the conditional c.d.f. (p.d.f.) of the second period demand with $F_{2 \mid d_{1}}\left(f_{2 \mid d_{1}}\right)$. According to Assumption 3 we get that $F_{2 \mid d_{1}}(x)=F_{Z}\left(\frac{2 x}{(M-1) d_{1}}\right)$.

It is useful to characterize the corresponding first best inventory decision at $t=1$. Let $x=$ $x_{\mathcal{R}}+x_{\mathcal{B}}$ denote the net expected cash flow extracted. It is easy to check that

$$
x= \begin{cases}d_{1}+s \cdot\left(y_{1}-y\right)+\int \min (u, y) f_{2 \mid d_{1}}(u) d u & 0 \leq y \leq y_{1} \\ d_{1}+c \cdot\left(y_{1}-y\right)+\int \min (u, y) f_{2 \mid d_{1}}(u) d u & y>y_{1},\end{cases}
$$

and thus

$$
\frac{d x}{d y}= \begin{cases}1-s-F_{2 \mid d_{1}}(y) & 0 \leq y \leq y_{1} \\ 1-c-F_{2 \mid d_{1}}(y) & y>y_{1}\end{cases}
$$

The first best inventory decision is thus given by

$$
y^{\mathrm{fb}}= \begin{cases}\min \left\{y_{1}, \frac{(M-1) d_{1}}{2} y_{s}\right\} & \text { under partial liq. only } \\ \max \left[\min \left\{y_{1}, \frac{(M-1) d_{1}}{2} y_{s}\right\}, \frac{(M-1) d_{1}}{2} y_{c}\right] & \text { under partial liq. and replenishment }\end{cases}
$$

where $y_{j} \stackrel{\text { def }}{=} F_{Z}^{-1}(1-j)$ for $j=c, s$. 
Proof of Lemma 6. Let $y_{\mathcal{P}}$ be the standalone optimal inventory decision of player $\mathcal{P} \in\{\mathcal{R}, \mathcal{B}\}$. Note that $\ell_{\mathcal{B}} \geq(>) \ell_{\mathcal{R}} \Leftrightarrow y_{\mathcal{B}} \leq(<) y_{\mathcal{R}}$. We first show that $y_{\mathcal{R}} \geq y^{\mathrm{fb}}$ and $y_{\mathcal{B}} \leq y^{\mathrm{fb}}$. We assume that $d_{1}<q$ throughout; for $d_{1} \geq q$ the firm stocks out and the players' actions are trivial.

Firm. Note that $x_{\mathcal{R}}=\mathbb{E}\left[\left(d_{1}+s \cdot\left(q-d_{1}-y\right)+\min \left\{y, D_{2}\right\}-R w\right)^{+} \mid D_{1}=d_{1}\right]$.

- For $0 \leq d_{1}<d_{\mathrm{S}}$, to break even at $t=2$ the inventory $y$ needs to exceed the minimum demand threshold $u_{\mathrm{B}}=(1-s)\left(d_{\mathrm{S}}-d_{1}\right)+s y$. Thus,

$$
x_{\mathcal{R}}= \begin{cases}0 & y \leq d_{\mathrm{S}}-d_{1} \\ \int_{u_{\mathrm{B}}}^{\infty}\left(\min \{u, y\}-u_{\mathrm{B}}\right) f_{2 \mid d_{1}}(u) d u & d_{\mathrm{S}}-d_{1}<y \leq y_{1},\end{cases}
$$

and

$$
\frac{d x_{\mathcal{R}}}{d y}= \begin{cases}0 & y \leq d_{\mathrm{S}}-d_{1} \\ \frac{d x}{d y}+s F_{2 \mid d_{1}}\left(u_{\mathrm{B}}\right) & d_{\mathrm{S}}-d_{1}<y \leq y_{1} .\end{cases}
$$

Consequently, we get that

$$
y_{\mathcal{R}} \begin{cases}>y^{\mathrm{fb}} & y^{\mathrm{fb}}<y_{1} \\ =y^{\mathrm{fb}} & \text { otherwise. }\end{cases}
$$

- For $d_{\mathrm{S}} \leq d_{1}<R w$, we have that $u_{\mathrm{B}} \leq y$. However, for small $y$ the threshold $u_{\mathrm{B}}$ could become negative, in which case there is no bankruptcy risk. In particular, we get that

$$
x_{\mathcal{R}}= \begin{cases}x-R w & y \leq \frac{1-s}{s}\left(d_{1}-d_{\mathrm{S}}\right) \\ \int_{u_{\mathrm{B}}}^{\infty}\left(\min \{u, y\}-u_{\mathrm{B}}\right) f_{2 \mid d_{1}}(u) d u & \frac{1-s}{s}\left(d_{1}-d_{\mathrm{S}}\right)<y \leq y_{1},\end{cases}
$$

and

$$
\frac{d x_{\mathcal{R}}}{d y}= \begin{cases}\frac{d x}{d y} & y \leq \frac{1-s}{s}\left(d_{1}-d_{\mathrm{S}}\right) \\ \frac{d x}{d y}+s F_{2 \mid d_{1}}\left(u_{\mathrm{B}}\right) & \frac{1-s}{s}\left(d_{1}-d_{\mathrm{S}}\right)<y \leq y_{1} .\end{cases}
$$

Consequently, we get that $y_{\mathcal{R}} \geq y^{\mathrm{fb}}$.

- For $d_{1} \geq R w$, there is no bankruptcy risk. Hence, $x_{\mathcal{R}}=x-R w$ and $y_{\mathcal{R}}=y^{\mathrm{fb}}$.

Bank. Note that $x_{\mathcal{B}}=\mathbb{E}\left[\min \left(d_{1}+s \cdot\left(q-d_{1}-y\right)+\min \left\{y, D_{2}\right\}, R w\right) \mid D_{1}=d_{1}\right]$.

- For $0 \leq d_{1}<d_{\mathrm{S}}, u_{\mathrm{B}}=(1-s)\left(d_{\mathrm{S}}-d_{1}\right)+s y$ is positive for any feasible $y$. Thus,

$$
x_{\mathcal{B}}= \begin{cases}x & y \leq d_{\mathrm{S}}-d_{1} \\ R w-\int_{0}^{u_{\mathrm{B}}}\left(u_{\mathrm{B}}-u\right) f_{2 \mid d_{1}}(u) d u & d_{\mathrm{S}}-d_{1}<y \leq y_{1},\end{cases}
$$


and

$$
\frac{d x_{\mathcal{B}}}{d y}= \begin{cases}\frac{d x}{d y} & y \leq d_{\mathrm{S}}-d_{1} \\ -s F_{2 \mid d_{1}}\left(u_{\mathrm{B}}\right) & d_{\mathrm{S}}-d_{1}<y \leq y_{1}\end{cases}
$$

Consequently, we get that $y_{\mathcal{B}}=\min \left\{d_{\mathrm{S}}-d_{1}, \frac{(M-1) d_{1}}{2} y_{s}\right\} \leq y^{\mathrm{fb}}$.

- For $d_{\mathrm{S}} \leq d_{1}<R w$, note first that if $\frac{1-s}{s}\left(d_{1}-d_{\mathrm{S}}\right)<y \leq y_{1}$, we have $\frac{d x_{\mathcal{B}}}{d y}=-s F_{2 \mid d_{1}}\left(u_{\mathrm{B}}\right) \leq 0$, so that $y_{\mathcal{B}} \leq \frac{1-s}{s}\left(d_{1}-d_{\mathrm{S}}\right)$ must hold. In the latter case, the threshold $u_{\mathrm{B}}$ is negative, in which case there is no bankruptcy risk, so without loss we have $y_{\mathcal{B}}=0<y^{\mathrm{fb}}$.

- For $d_{1} \geq R w$, there is no bankruptcy risk. Hence, without loss we again have $y_{\mathcal{B}}=0<y^{\mathrm{fb}}$.

To finish the proof, note that for $d_{1}<d_{\mathrm{S}}$, if $y^{\mathrm{fb}}=y_{1}$ we have that $y_{\mathcal{B}} \leq d_{\mathrm{S}}-d_{1}<q-d_{1}=$ $y_{1}=y_{\mathcal{R}}$. If $y^{\mathrm{fb}}<y_{1}$, we have that $y_{\mathcal{B}} \leq y^{\mathrm{fb}}<y_{\mathcal{R}}$.

Proof of Lemma 7. If $\ell_{\mathcal{R}} \geq \ell_{\mathrm{C}}$, then the firm follows its standalone optimal policy and the covenant does not get violated, $\ell_{\mathcal{R}}^{\star}=\ell_{\mathcal{R}} \geq \ell_{\mathrm{C}}$.

If $\ell_{\mathcal{R}}<\ell_{\mathrm{C}}$, following the firm's standalone optimal policy would lead to a covenant violation. In such case, the bank will enforce her optimal decision. According to our analysis in the proof of Lemma 6 , for $d \geq d_{\mathrm{S}}$ we have $y_{\mathcal{B}}=0$. Thus, the firm's cash flow, in anticipation of the bank's, will be

$$
x_{\mathcal{R}}= \begin{cases}x_{\mathcal{R}} & 0 \leq y \leq y_{\mathrm{C}} \\ \left.x_{\mathcal{R}}\right|_{y=0} & y>y_{\mathrm{C}}\end{cases}
$$

where $y_{\mathrm{C}}$ is the inventory position that corresponds to $\ell_{\mathrm{C}}$. According to our analysis in the proof of Lemma 6 , for $d \geq d_{\mathrm{S}}$ we also have that

$$
\left.\frac{d x_{\mathcal{R}}}{d y}\right|_{y=0}=\left.\frac{d x}{d y}\right|_{y=0}=1-s>0
$$

Thus, the firm would always prefer an inventory position that does not violate the covenant and $y_{\mathcal{R}} \leq y_{\mathrm{C}}$, i.e., $\ell_{\mathcal{R}}^{\star} \geq \ell_{\mathrm{C}}$. To conclude the proof, it is easy to check that $y_{\mathrm{C}} \geq 0$ for $d \geq d_{\mathrm{S}}$.

Proof of Theorem 5. We first characterize the threshold $\bar{x}_{0}$ by following similar steps as in the proof of Theorem 4. Consider the corresponding interest rate $R^{\mathrm{fb}}$ defined as in Section D.2 and let $q_{\mathrm{S}}^{\mathrm{fb}} \stackrel{\text { def }}{=} \frac{R^{\mathrm{fb}} x_{0}}{R^{\mathrm{fb}} c-s}$; this quantity plays the same role as $q_{\mathbb{D}}^{\mathrm{fb}}$ in the proof of Theorem 4 . In particular, as in (19), note that

$$
\operatorname{sgn}\left(\frac{d q_{\mathrm{S}}^{\mathrm{fb}}}{d x_{0}}\right)=\operatorname{sgn}\left[\frac{d}{d x_{0}}\left(\frac{R^{\mathrm{fb}} x_{0}}{R^{\mathrm{fb}} c-s}\right)\right]=\operatorname{sgn}\left[R^{\mathrm{fb}}\left(R^{\mathrm{fb}} c-s\right)-x_{0} s \frac{\partial R^{\mathrm{fb}}}{\partial x_{0}}\right]>0
$$

and thus $q_{\mathrm{S}}^{\mathrm{fb}}$ is increasing in $x_{0}$. Let $\bar{x}_{0}$ be such that $q^{\mathrm{fb}}>q_{\mathrm{S}}^{\mathrm{fb}}$ if and only if $x_{0}<\bar{x}_{0}$. 
For the sake of contradiction, assume now that $x_{0}<\bar{x}_{0}$ and for some contract $\kappa \in \mathbb{K}^{\mathrm{F}}$ we have that $A(\kappa)=0$. Thus, the first best actions $q^{\mathrm{fb}}$ and $y^{\mathrm{fb}}$ are followed in equilibrium.

Since $x_{0}<\bar{x}_{0}$, we have that $q^{\mathrm{fb}}>q_{\mathrm{S}}^{\mathrm{fb}}$, which implies that $d_{\mathrm{S}}>0$. Consider now the problem that the firm solves at $t=1$ for a demand level $d_{1}<d_{\mathrm{S}}$ such that $d_{\mathrm{S}}-d_{1}<\frac{(M-1) d_{1}}{2} y_{s}<q-d_{1}$. Based on our analysis in the proof of Lemma 6 , we get that $y_{\mathcal{B}}=d_{\mathrm{S}}-d_{1}<\frac{(M-1) d_{1}}{2} y_{s}=y^{\mathrm{fb}}$. Thus, since $y^{\mathrm{fb}}$ is followed in equilibrium, we get that the covenant is not violated, $d_{1}+s\left(q-d_{1}-\frac{(M-1) d_{1}}{2} y_{s}\right) \leq \delta$. Similarly, since $y_{\mathcal{R}}>y^{\mathrm{fb}}$, the equilibrium action also implies that the covenant is in fact binding, $d_{1}+s\left(q-d_{1}-\frac{(M-1) d_{1}}{2} y_{s}\right)=\delta$.

Consider now the problem that the firm solves at $t=1$ for a demand level $d_{1}^{\prime}=d_{1}-\epsilon$, for $\epsilon>0$ small enough such that $d_{1}^{\prime}<d_{\mathrm{S}}$ and $d_{\mathrm{S}}-d_{1}^{\prime}<\frac{(M-1) d_{1}^{\prime}}{2} y_{s}<q-d_{1}^{\prime}$ still hold. All arguments made above still hold. However, the covenant is not binding since

$$
d_{1}^{\prime}+s\left(q-d_{1}^{\prime}-\frac{(M-1) d_{1}^{\prime}}{2} y_{s}\right)=d_{1}+s\left(q-d_{1}-\frac{(M-1) d_{1}}{2} y_{s}\right)-\left(1-s-s \frac{(M-1)}{2} y_{s}\right) \epsilon \neq \delta
$$

a contradiction.

Proof of Theorem 6. We use notation consistent with the proof of Proposition 6. Let $x(q ; y)$ be the net expected revenues at the end of the game, generated by sales and possible inventory liquidation, under an order quantity $q$ and an inventory liquidation policy $y$ at $t=1$. We can express the firm's profits as the net expected revenues minus inventory purchasing costs minus the bank's profits, that is, for any $\kappa \in \mathbb{K}^{\mathrm{B}}$

$$
\pi_{\mathcal{R}}(q, \kappa)=x\left(q ; y_{\kappa, q}\right)-c q-\pi_{\mathcal{B}}(q, \kappa)=x\left(q ; y_{\kappa, q}\right)-c q
$$

where $y_{\kappa, q}$ is the equilibrium inventory liquidation policy followed under quantity $q$ and contract $\kappa$.

We first show that the equilibrium inventory liquidation policy under $\kappa^{\star}=\left(\alpha^{\star}, \beta^{\star}\right) \in \mathbb{K}^{\mathrm{B}}$ is first best, i.e., $y_{\kappa^{\star}, q}=y^{\mathrm{fb}}$. If the firm stocks out at $t=1$, i.e., $d_{1} \geq q$, all policies are trivial. Thus, we only consider the case of $d_{1}<q$. Note that the covenant condition is equivalent to

$$
\underbrace{d_{1}+s\left(y_{1}-y\right)}_{\text {cash }}+\alpha^{\star} \underbrace{s y}_{\text {inventory }} \geq \beta^{\star} \Leftrightarrow y \leq \frac{(M-1) d_{1}}{2} y_{s} .
$$

Based on our analysis in the proof of Lemma 6, we get that:

- For $d_{1}<d_{\mathrm{S}}$,

- for $y_{1} \leq \frac{(M-1) d_{1}}{2} y_{s}$, we get that $y_{\mathcal{R}}=y^{\mathrm{fb}}=y_{1}$ and since the covenant does not get violated for this policy, $y_{\kappa^{\star}, q}=y^{\mathrm{fb}}$.

- for $d_{\mathrm{S}}-d_{1}<\frac{(M-1) d_{1}}{2} y_{s}<y_{1}$, we get that $y^{\mathrm{fb}}=\frac{(M-1) d_{1}}{2} y_{s}<y_{1}$ and thus $y_{\mathcal{R}}>\frac{(M-1) d_{1}}{2} y_{s}$, i.e., the covenant would get violated for $y_{\mathcal{R}}$. In that case, we have $y_{\mathcal{B}}=d_{\mathrm{S}}-d_{1}$, which 
implies that the firm will be bankrupt if the covenant is violated. Thus, it would prefer any $0 \leq y \leq y^{\mathrm{fb}}$ to avert violation. Since, $\frac{d x_{\mathcal{R}}}{d y} \geq 0$ for all such $y$, we get that $y_{\kappa^{\star}, q}=y^{\mathrm{fb}}$. - for $\frac{(M-1) d_{1}}{2} y_{s} \leq d_{\mathrm{S}}-d_{1}$, the firm is bankrupt if $y \leq d_{\mathrm{S}}-d_{1}$ and the covenant is violated otherwise. Hence, without loss the firm is indifferent and takes no action leading to a covenant violation. Then, the bank's policy is followed in equilibrium and $y_{\kappa^{\star}, q}=y_{\mathcal{B}}=$ $\frac{(M-1) d_{1}}{2} y_{s}=y^{\mathrm{fb}}$.

- For $d_{\mathrm{S}} \leq d_{1} \leq R w$, a covenant violation would transfer control rights to the bank, in which case $y_{\mathcal{B}}=0$ would be followed. Specifically,

- for $y_{1} \leq \frac{(M-1) d_{1}}{2} y_{s}$, we get that $\frac{d x_{\mathcal{R}}}{d y} \geq 0$ for all $0 \leq y \leq y_{1}$. Thus, $y_{\mathcal{R}}=y_{1}$ and since the covenant does not get violated for this policy, $y_{\kappa^{\star}, q}=y_{1}=y^{\mathrm{fb}}$.

- for $\frac{(M-1) d_{1}}{2} y_{s}<y_{1}$, since $\frac{d x_{\mathcal{R}}}{d y} \geq 0$ for all $0 \leq y \leq \frac{(M-1) d_{1}}{2} y_{s}$ we get that $y_{\kappa^{\star}, q}=$ $\frac{(M-1) d_{1}}{2} y_{s}=y^{\mathrm{fb}}$.

- For $d_{1}>R w$, we have that $y_{\mathcal{R}}=y^{\mathrm{fb}} \leq \frac{(M-1) d_{1}}{2} y_{s}$ and since the covenant does not get violated for this policy, $y_{\kappa^{\star}, q}=y^{\mathrm{fb}}$.

We now have

$$
\begin{aligned}
V_{\mathcal{R}}\left(\kappa^{\star}\right) & =\max _{q}\left\{x\left(q ; y_{\kappa^{\star}, q}\right)-c q\right\} \\
& \geq x\left(q^{\mathrm{fb}} ; y_{\kappa^{\star}, q^{\mathrm{fb}}}\right)-c q^{\mathrm{fb}} \\
& =x\left(q^{\mathrm{fb}} ; y^{\mathrm{fb}}\right)-c q^{\mathrm{fb}} \\
& =V^{\mathrm{fb}} \\
& \geq \sup _{\kappa \in \mathbb{K}^{\mathrm{B}}} V_{\mathcal{R}}(\kappa)=V_{\mathcal{R}}\left(\kappa^{\star}\right) .
\end{aligned}
$$

Thus,

$$
\min _{\kappa \in \mathbb{K}^{\mathrm{B}}} A(\kappa) \leq A\left(\kappa^{\star}\right)=\frac{V^{\mathrm{fb}}-V_{\mathcal{R}}\left(\kappa^{\star}\right)}{V^{\mathrm{fb}}}=\frac{V^{\mathrm{fb}}-V^{\mathrm{fb}}}{V^{\mathrm{fb}}}=0
$$

which completes the first part of the proof.

The comparative statics results for $\alpha^{\star}=1-\frac{2(1-s)}{s(M-1) F_{Z}^{-1}(1-s)}$ are immediate, since the denominator is increasing in $M$ and in $Z$ (in the usual stochastic order). Furthermore, since $q^{\mathrm{fb}}$ is increasing in $s, M, D_{1}$ and $Z$ (in the usual stochastic order), so is $\beta^{\star}=s q^{\mathrm{fb}}$.

Proof of Theorem 7. We first deal with the case where reordering is financed exclusively with cash from the lockbox account. The proof uses a similar stream of arguments as the proof of Theorem 6 . Thus, if $y_{\kappa, q}$ is the equilibrium inventory adjustment policy followed under quantity 
$q$ and contract $\kappa$, it suffices to show that the equilibrium inventory liquidation policy under $\kappa^{\star}=$ $\left(\alpha^{\star}, \beta^{\star}\right) \in \mathbb{K}^{\mathrm{B}}$ is first best, i.e., $y_{\kappa^{\star}, q}=y^{\mathrm{fb}}$.

Case 1: Suppose that $y^{\mathrm{fb}}=\min \left\{y_{1}, \frac{(M-1) d_{1}}{2} y_{s}\right\}$. Note that in that case we get that $\frac{(M-1) d_{1}}{2} y_{c} \leq$ $y_{1}$, so that $y_{1}=q-d_{1}>0$. Then, the withdrawal limit of the firm at $t=1$ also simplifies to

$$
\phi b-c q=\left(\frac{(M-1) d_{1}}{2} y_{c}-y_{1}\right) c \leq 0
$$

so that no replenishment can take place. Hence, the firm follows an inventory adjustment policy according to our analysis in Theorem 6 and $y_{\kappa^{\star}, q}=y^{\mathrm{fb}}$.

Case 2: Suppose that $y^{\mathrm{fb}}=\frac{(M-1) d_{1}}{2} y_{c}$. Thus, we have that $y_{1}=q-d_{1} \leq \frac{(M-1) d_{1}}{2} y_{c}<\frac{(M-1) d_{1}}{2} y_{s}$. We first argue that the covenant is never violated in this case. In particular, by the withdrawal limit, we get that $c\left(y-y_{1}\right) \leq \phi b-c q=\left(\frac{(M-1) d_{1}}{2} y_{c}-y_{1}\right) c$ and thus $y \leq \frac{(M-1) d_{1}}{2} y_{c}<\frac{(M-1) d_{1}}{2} y_{s}$ and the covenant is never violated.

We next argue that no partial liquidation will be performed in equilibrium, $y_{\kappa^{\star}, q} \geq y_{1}$. Based on our analysis in the proof of Lemma 6 , we get that $\frac{d x_{\mathcal{R}}}{d y} \geq 0$ for all $y \leq y_{1}$, since $y_{1}<\frac{(M-1) d_{1}}{2} y_{s}$. In conjuction with the covenant not being violated we get that $y_{\kappa^{\star}, q} \geq y_{1}$.

By combining the two facts above, we conclude that the equilibrium policy will be dictated by the firm, which would effectively act by maximizing its payoff $x_{\mathcal{R}}$ over $y_{1} \leq y \leq y^{\mathrm{fb}}$. Similarly to our analysis in the proof of Lemma 6 , it can be checked that $u_{\mathrm{B}}=R w-\min \left\{q, d_{1}\right\}-c\left(y_{1}-y\right)$ is the minimum second-period demand threshold for the firm to break even. For $u_{\mathrm{B}}<0$, there is no bankruptcy risk, $x_{\mathcal{R}}=x-R w$ and the firm follows $y_{\mathcal{R}}=y^{\mathrm{fb}}$. For $u_{\mathrm{B}} \geq 0$, we get that

$$
x_{\mathcal{R}}=\int_{u_{\mathrm{B}}}^{y}\left(u-u_{\mathrm{B}}\right) f_{2 \mid d_{1}}(u) d u+\int_{y}^{\infty}\left(y-u_{\mathrm{B}}\right) f_{2 \mid d_{1}}(u) d u,
$$

and

$$
\frac{d x_{\mathcal{R}}}{d y}=\frac{d x}{d y}+c F_{2 \mid d_{1}}\left(u_{\mathrm{B}}\right) \geq \frac{d x}{d y} \geq 0, \quad \forall y_{1} \leq y \leq y^{\mathrm{fb}} .
$$

Consequently, $y_{\kappa^{\star}, q}=y^{\mathrm{fb}}$.

Finally, we discuss the case where additional funds are required to place the order. In this case, there will be no agency costs, since the firm will act as a newsvendor, who has no dynamic decisions left. Thus, first best decisions will always be followed, and the proof is complete.

\section{D.4 Monopoly}

For the purposes of this section, it will help to think about the covenant in terms of a cash-flowto-debt ratio threshold $\gamma \stackrel{\text { def }}{=} \frac{\delta}{R w}$, instead of the cash flow threshold $\delta$. This is without loss (see Section 3.3). We first introduce some simplifying notation. Let $\left(R^{\star}, \gamma^{\star}\right)$ denote the optimal contract offered by the bank in equilibrium, and let $q^{\star}(\gamma)$ denote the firm's optimal response to a contract 
$\left(R^{\star}, \gamma\right)$, i.e., where only the covenant threshold is changing, and the interest rate is fixed to the optimal value. For simplicity, also let $q^{\star}\left(\gamma^{\star}\right)=q^{\star \star}$.

Several quantities of interest in the proof depend on the interest rate $R$ - for instance, the threshold $q_{\mathbb{D}}$ defined in (2), and the values $\underline{d}(q)$ and $\bar{d}(q)$, defined in Lemma 5. To avoid introducing unnecessary notation, for all the proofs in the rest of the section, we use the implicit understanding that any such quantity is calculated under $R=R^{\star}$, i.e., using the optimal interest rate for the bank in equilibrium. This should not create any confusion, since all the arguments presented will not rely on changing $R$. For simplicity, we also introduce $\gamma_{\min } \stackrel{\text { def }}{=} \frac{d\left(q^{\star \star}\right)}{R^{\star}\left(c q^{\star \star}-x_{0}\right)}$ and $\gamma_{\max } \stackrel{\text { def }}{=} \frac{\bar{d}\left(q^{\star \star}\right)}{R^{\star}\left(c q^{\star \star}-x_{0}\right)}$.

We let $\Delta X_{\mathcal{P}}\left(q, d_{1}\right) \stackrel{\text { def }}{=} X_{\mathcal{P}, \mathscr{L}}\left(q, d_{1}\right)-\mathbb{E}\left[X_{\mathcal{P}, \mathscr{C}}\left(q, d_{1}, D_{2}\right) \mid D_{1}=d_{1}\right]$ denote the expected revenue difference between liquidation and continuation of player $\mathcal{P} \in\{\mathcal{R}, \mathcal{B}\}$, when the bank contract is $\left(R^{\star}, \gamma\right)$, and the firm orders a generic $q$. If $q$ results in liquidation conflict, note that

$$
\Delta X_{\mathcal{R}}(q, \underline{d}(q))=0
$$

by the definition of $\underline{d}(q)$ (see Lemma 5 ).

Proof of Theorem 8. It suffices to show this result for financial covenants, as borrowing base covenants subsume these as special cases in our setting.

When there is no liquidation conflict, by Lemma 5, the disagreement region is empty, and the equilibrium liquidation policy followed is $\ell_{\mathcal{R}}\left(q^{\star \star}, d\right)$, according to Lemma 2. As such, a contract $\left(R^{\star}, 0\right)$ would result in exactly the same liquidation policy in equilibrium, and would also yield $q^{\star}(0)=q^{\star \star}$. Without loss, then, no covenant is needed in equilibrium.

For the converse, it suffices to show that, when there is liquidation conflict, $\gamma^{\star}>0$. For the purposes of deriving a contradiction, assume that $\gamma^{\star}=0$. Then, $q^{\star}(0)$ is given by the first-order optimality condition (FOC) of $\pi_{\mathcal{R}}$.

We first argue for cases where the bank follows a threshold policy (see Lemma 4). By the implicit function theorem applied to the derivative of the FOC with respect to $\gamma$, we get that

$$
\left.\frac{\partial^{2} \pi_{\mathcal{R}}}{\partial q \partial \gamma}\right|_{q^{\star}(\gamma)}+\left.\frac{\partial^{2} \pi_{\mathcal{R}}}{\partial q^{2}}\right|_{q^{\star}(\gamma)} \frac{d q^{\star}(\gamma)}{d \gamma}=0 .
$$

We now evaluate the terms above at $\left(q^{\star}\left(\gamma_{\min }\right), \gamma_{\text {min }}\right)$. Recall that $q^{\star}\left(\gamma_{\text {min }}\right)=q^{\star}(0)$. By our standing assumption,

$$
\left.\frac{\partial^{2} \pi_{\mathcal{R}}}{\partial q^{2}}\right|_{q^{\star}\left(\gamma_{\text {min }}\right)}=\left.\frac{\partial^{2} \pi_{\mathcal{R}}}{\partial q^{2}}\right|_{q^{\star}(0)}<0 .
$$

To evaluate the mixed derivative above, we express the firm's expected profits, for $q>q_{\mathbb{D}}$ and $\gamma_{\min } \leq \gamma \leq \gamma_{\max }$, as $\pi_{\mathcal{R}}(q, \gamma)=\pi_{\mathcal{R}}(q, 0)+\int_{\underline{d}(q)}^{\delta} \Delta X_{\mathcal{R}}(q, u) f_{1}(u) d u$, where $\delta$ is the covenant demand threshold. To see this, note that in the disagreement region where the covenant is breached, $(\underline{d}(q), \delta)$, the firm would have otherwise continued for $\gamma=0$, whereas it faces liquidation for $\gamma$. We 
then have

$$
\left.\frac{\partial^{2} \pi_{\mathcal{R}}}{\partial q \partial \gamma}\right|_{\substack{q^{\star}\left(\gamma_{\min }\right), \gamma_{\min }}}=\left.\left[\left(\frac{\partial}{\partial q} \Delta X_{\mathcal{R}}(q, \delta)\right) f_{1}(\delta) \frac{\partial \delta}{\partial \gamma}\right]\right|_{\substack{q^{\star}\left(\gamma_{\min }\right), \gamma_{\min }}}+\left.\left[\Delta X_{\mathcal{R}}(q, \delta) \frac{\partial}{\partial q}\left(f_{1}(\delta) \frac{\partial \delta}{\partial \gamma}\right)\right]\right|_{\substack{q^{\star}\left(\gamma_{\min }\right) \\ \gamma_{\min }}}
$$

Note that $\delta$ evaluated at $\left(q^{\star}\left(\gamma_{\min }\right), \gamma_{\min }\right)$ is equal to $\underline{d}\left(q^{\star}\left(\gamma_{\min }\right)\right)$. Therefore, by equation (20), the second term above is zero.

To determine the sign of the first term, note that $\delta$ is increasing in $\gamma$, and $f_{1}$ is positive. Thus, at the point $\left(q^{\star}\left(\gamma_{\min }\right), \gamma_{\min }\right)$, the sign of $\frac{\partial^{2} \pi_{\mathcal{R}}}{\partial q \partial \gamma}$ is the same as the sign of $\frac{\partial \Delta X_{\mathcal{R}}(q, \delta)}{\partial q}$. To evaluate the latter, we apply the implicit function theorem to equation (20) to obtain:

$$
\left.\frac{\partial \Delta X_{\mathcal{R}}}{\partial q}\right|_{\substack{q^{\star}\left(\gamma_{\text {min }}\right) \\ \underline{d}\left(q^{\star}\left(\gamma_{\min }\right)\right)}}=-\left.\left.\frac{\partial \underline{d}(q)}{\partial q}\right|_{q^{\star}\left(\gamma_{\min }\right)} \frac{\partial \Delta X_{\mathcal{R}}}{\partial d}\right|_{\substack{q^{\star}\left(\gamma_{\min }\right) \\ \gamma_{\min }}}
$$

Since $\underline{d}$ is increasing in $q$ (see Lemma 5), the sign of the first multiplier in the right-hand side is positive. By Proposition 8, the sign of the second multiplier in the right-hand side is negative. We conclude that the sign of $\frac{\partial^{2} \pi_{\mathcal{R}}}{\partial q \partial \gamma}$ at $\left(q^{\star}\left(\gamma_{\min }\right), \gamma_{\min }\right)$ is positive.

By combining the above fact with (21) and (22), we get that

$$
\left.\frac{d q^{\star}(\gamma)}{d \gamma}\right|_{\gamma_{\min }} \geq 0
$$

We now focus on the bank's expected profits. For $q>q_{\mathbb{D}}$ and $\gamma_{\min } \leq \gamma \leq \gamma_{\max }$, we can express them as $\pi_{\mathcal{B}}(q, \gamma)=\pi_{\mathcal{B}}(q, 0)+\int_{\underline{d}(q)}^{\delta} \Delta X_{\mathcal{B}}(q, u) f_{1}(u) d u$. Thus, $\frac{\partial \pi_{\mathcal{B}}(q, \gamma)}{\partial \gamma}=R^{\star}\left(c q-x_{0}\right) \Delta X_{\mathcal{B}}(q, \delta) f_{1}(\delta)$. By the implicit function theorem we also get $\frac{d \pi_{\mathcal{B}}\left(q^{\star}(\gamma), \gamma\right)}{d \gamma}=\left.\frac{\partial \pi_{\mathcal{B}}(q, \gamma)}{\partial \gamma}\right|_{q^{\star}(\gamma)}+\left.\frac{d q^{\star}(\gamma)}{d \gamma} \frac{\partial \pi_{\mathcal{B}}(q, \gamma)}{\partial q}\right|_{q^{\star}(\gamma)}$. Thus, by combining the two equations above, we get

$$
\left.\frac{d \pi_{\mathcal{B}}\left(q^{\star}(\gamma), \gamma\right)}{d \gamma}\right|_{\gamma_{\text {min }}}=\underbrace{R^{\star}\left(c q^{\star}(0)-x_{0}\right) \Delta X_{\mathcal{B}}\left(q^{\star}(0), \delta_{\min }\right) f_{1}\left(\delta_{\min }\right)}_{>0}+\left.\underbrace{\left.\frac{d q^{\star}(\gamma)}{d \gamma}\right|_{\gamma_{\min }}}_{\geq 0} \underbrace{\frac{\partial \pi_{\mathcal{B}}(q, \gamma)}{\partial q}}_{\geq 0}\right|_{q^{\star}(0)}>0,
$$

Note that the first term above is strictly positive due to the existence of liquidation conflict (also see Proof of Lemma 2). The second term is positive by (23). To argue that the third term is positive, assume for the sake of contradiction that it is negative. Then, the bank can strictly increase its profit by slightly increasing the interest rate $R^{\star}$ (as the firm's quantity would slightly decrease as a result), thus contradicting optimality of the contract $\left(R^{\star}, 0\right)$. This shows that the assumed contract $\left(R^{\star}, 0\right)$ is suboptimal for the bank, since she can increase her profits by including a covenant.

We now argue for the case when the bank does not follow a threshold policy, i.e., when $q^{\star}(0)>$ $q_{\mathcal{B}}, s<s_{\mathcal{B}}$. By using our results from Lemma 2 and Lemma 4 , note that for $d<d^{\mathrm{fb}}$, it is optimal for the firm to either liquidate or continue, and for the bank to liquidate. Thus, in equilibrium, 
$\delta^{\star} \geq d^{\mathrm{fb}}>0$, which implies that a covenant is needed.

Finally, as in Lemma 3, one can show that when the optimal liquidation policy of the bank is followed, sufficient leverage (or, equivalently, liquidation conflict) is a necessary condition for bankruptcy risk in non-shrinking markets, and neither necessary, nor sufficient in shrinking markets.

\section{D.4.1 Auxiliary Results}

Proposition 8. When there is liquidation conflict and the bank is following a threshold policy, $\frac{\partial \Delta X_{\mathcal{R}}}{\partial d}$ evaluated at $\left(q^{\star}\left(\gamma_{\text {min }}\right), \underline{d}\left(q^{\star}\left(\gamma_{\text {min }}\right)\right)\right)$ is negative.

Proof. For simplicity of notation, we denote $q^{\star}\left(\gamma_{\min }\right)$ with a generic $q$. We distinguish two cases. For $M \geq \tilde{M}$, since the bank follows a non-threshold liquidation policy, $\underline{d}(q)=d_{\mathcal{R}}(q)$ (see Lemma 5 ). Since the firm always prefers continuation for $d \geq \frac{q}{M}$ (see Proposition 4), and prefers liquidation for $d<d^{\mathrm{fb}}(q)$, we must have $\underline{d}(q)<\frac{q}{M}$. Therefore, $x_{\mathcal{R}, \mathscr{C}}(q, d)=\frac{1}{2}(M d-w R)$. By combining this with the liquidation payoffs given by (8), we have that

$$
\Delta X_{\mathcal{R}}(q, d)= \begin{cases}-\frac{1}{2}(M d-w R), & \text { if } d<d_{\mathrm{S}} \\ s q+(1-s) d-w R-\frac{1}{2}(M d-w R), & \text { otherwise }\end{cases}
$$

As such, $\frac{\partial \Delta X_{\mathcal{R}}}{\partial d}$ is equal to $-\frac{M}{2}$ or $\frac{\tilde{M}-M}{2}$, and is therefore negative.

For $M<\tilde{M}, \underline{d}(q)=\frac{w R}{M}<\frac{q}{M}$. As such, the liquidation and continuation profits are exactly the same as above. Note, however, that $\underline{d}(q)<d_{\mathrm{S}}$, by $(15 \mathrm{e})$ in Lemma 5 . Thus, $\frac{\partial \Delta X_{\mathcal{R}}}{\partial d}$ is equal to $-\frac{M}{2}$, and the proof is complete.

\section{D.5 Robustness of Non-Threshold Policies}

Proof of Theorem 9. Since $q$ is fixed, we no longer explicitly show the dependency of the various functions on $q$. When the first period demand is $D_{1}=d$, the firm's expected liquidation and continuation payoffs are respectively given by:

$$
\begin{aligned}
& x_{\mathcal{R}, \mathscr{L}}(d)=X_{\mathcal{R}, \mathscr{L}}(q, d)= \begin{cases}0, & \text { if } d \leq d_{\mathrm{S}} \\
(1-s) d+s q-R w, & \text { if } d_{\mathrm{S}}<d \leq q \\
q-R w, & \text { if } q<d .\end{cases} \\
& x_{\mathcal{R}, \mathscr{C}}(d)=\mathbb{E}_{D_{2} \mid D_{1}=d}\left[X_{\mathcal{R}, \mathscr{C}}\left(q, d, D_{2}\right)\right]=\mathbb{E}_{D_{2} \mid D_{1}=d}\left[\left(\min \left(d+D_{2}, q\right)-R w\right)^{+}\right] .
\end{aligned}
$$

Consider the function $g(y) \stackrel{\text { def }}{=}(\min (y, q)-R w)^{+}$. Since $R w \leq q$, note that $g$ is identically zero for $y \leq R w$, then increases linearly, with a slope of 1 , and then becomes constant, equal to $q-R w$, for 
$y \geq q$. In particular, $g(y)$ is convex-concave, ${ }^{21}$ and increasing. Since $x_{\mathcal{R}, \mathscr{C}}(d)=\mathbb{E} g\left(d+D_{2}\right)$, and $D_{2}$ has a distribution with log-concave density, the function $x_{\mathcal{R}, \mathscr{C}}(d)$ will also be convex-concave and increasing in $d$ (Porteus 2002).

Consider the two functions $x_{\mathcal{R}, \mathscr{L}}(d)$ and $x_{\mathcal{R}, \mathscr{C}}(d)$. Both are positive and increasing on $[0, q)$, and satisfy $x_{\mathcal{R}, \mathscr{L}}(d)=x_{\mathcal{R}, \mathscr{C}}(d)=q-R w, \forall d \geq q$. It can be readily checked that these functions can have at most two intersection points on $(0, q)$, based on the convex-concave structure of $x_{\mathcal{R}, \mathscr{C}}$. Furthermore, if there exists $0<\xi<q$ such that $x_{\mathcal{R}, \mathscr{L}}\left(\xi_{-}\right)>x_{\mathcal{R}, \mathscr{C}}\left(\xi_{-}\right)$and $x_{\mathcal{R}, \mathscr{L}}\left(\xi_{+}\right)<x_{\mathcal{R}, \mathscr{C}}\left(\xi_{+}\right)$, then $x_{\mathcal{R}, \mathscr{L}}(d)<x_{\mathcal{R}, \mathscr{C}}(d), \forall d \in(\xi, q)$. The conclusion of the theorem concerning the firm follows.

The proof for the bank follows in a similar fashion, by recognizing that the expected profits from liquidation and continuation, i.e.,

$$
\begin{aligned}
x_{\mathcal{B}, \mathscr{L}}(d) & =X_{\mathcal{B}, \mathscr{L}}(d)=\min \left\{R w(q), \min (d, q)+s(q-d)^{+}\right\}, \\
x_{\mathcal{B}, \mathscr{C}}(q, d) & =\mathbb{E}\left[X_{\mathcal{B}, \mathscr{C}}\left(q, d, D_{2}\right)\right]=\mathbb{E}\left[\min \left\{R w(q), \min \left(d+D_{2}, q\right)\right\}\right],
\end{aligned}
$$

are concave increasing on $d \in(0, R w)$, and can have at most two intersection points on this interval. Note that the PF2 requirement is not needed, since the bank's profits from continuation are always concave increasing, for any second period demand distribution.

Proof of Proposition 1. (a) Note first that, since $D_{1}>\frac{x_{0}}{c-\frac{s M}{M+s-1}} \geq \frac{x_{0}}{c}$ holds almost surely, the firm will prefer to be leveraged, and to order a quantity $q>\frac{x_{0}}{c-\frac{s M}{M+s-1}}$, for any interest rate $R \leq \frac{1}{c}$. In particular, since the equilibrium interest rate always satisfies this inequality, we have $q^{\star}>\frac{x_{0}}{c-\frac{s M}{M+s-1}}$.

Recall that $s_{\mathbb{D}}^{2}=\frac{R c(M-1)}{M-R c}, q_{\mathbb{D}}^{2}=\frac{R x_{0}}{R c-\frac{s M}{M+s-1}}$. As such, the conditions in the proposition ensure that $s<s_{\mathbb{D}}^{2}$ and $q_{\mathbb{D}}^{2}>0$ holds at $R \geq 1$. Since $s_{\mathbb{D}}^{2}$ is increasing in $R$, and $q_{\mathbb{D}}^{2}$ is decreasing in $R$, we immediately see that $s<s_{\mathbb{D}}^{2}$ and $q^{\star}>q_{\mathbb{D}}^{2}$ must hold in equilibrium. Since $M<\tilde{M}$, this implies that the firm will always be sufficiently leveraged in equilibrium, and will follow non-threshold policies (see Lemma 2).

(b) As above, we must have that, in equilibrium, the firm prefers to be leveraged, and $q^{\star}>$ $\frac{(M-1+2 s) x_{0}}{c(M-1+2 s)-2 M s}>\frac{x_{0}}{c}>0$.

Recall that $s_{\mathcal{B}}=\frac{(M-1) c R}{2(M-c R)}$ and $q_{\mathcal{B}}=\frac{R(M-1+2 s) x_{0}}{c R(M-1+2 s)-2 M s}$. The conditions in the proposition ensure that $s<s_{\mathcal{B}}$ and $q^{\star}>q_{\mathcal{B}}$ holds for $R=1$. Since $s_{\mathcal{B}}$ is increasing in $R$, and $q_{\mathcal{B}}$ is decreasing in $R$, we have that $s<s_{\mathcal{B}}$ and $q^{\star}>q_{\mathcal{B}}$ must also hold for the optimal interest rate, proving that the bank will always follow non-threshold policies in equilibrium (see Lemma 4).

\footnotetext{
${ }^{21}$ Following standard terminology, we call a function $g: \mathbb{R} \rightarrow \mathbb{R}$ convex-concave if it is convex on $(-\infty, a)$ and convex on $(a, \infty)$, for some $a \in \mathbb{R}$ (see, e.g., Porteus (2002)).
} 